\title{
Riemann surfaces for KPZ with periodic boundaries
}

\author{
Sylvain Prolhac \\ Laboratoire de Physique Théorique, IRSAMC, UPS, Université de Toulouse, France \\ sylvain.prolhac@irsamc.ups-tlse.fr
}

November 15, 2019

\begin{abstract}
The Riemann surface for polylogarithms of half-integer index, which has the topology of an infinite dimensional hypercube, is studied in relation to onedimensional KPZ universality in finite volume. Known exact results for fluctuations of the KPZ height with periodic boundaries are expressed in terms of meromorphic functions on this Riemann surface, summed over all the sheets of a covering map to an infinite cylinder. Connections to stationary large deviations, particle-hole excitations and $\mathrm{KdV}$ solitons are discussed.
\end{abstract}

\section{Contents}

\begin{tabular}{lll}
\hline 1 & Introduction & 3
\end{tabular}

2 KPZ fluctuations and Riemann surfaces 4

2.1 Riemann surfaces $\check{\mathcal{R}}$ and $\mathcal{R}^{\Delta} \quad 4$

$\begin{array}{lll}2.2 & \text { Flat initial condition } & 5\end{array}$

\begin{tabular}{lll}
\hline 2.3 & Sharp wedge initial condition & 7
\end{tabular}

$\begin{array}{lll}2.4 & \text { Stationary initial condition } & 8\end{array}$

\begin{tabular}{|lll}
2.5 & Multiple-time statistics with sharp wedge initial condition & 8
\end{tabular}

$\begin{array}{lll}2.6 & \text { Discussion } & 9\end{array}$

\begin{tabular}{|lll}
2.6 .1 & Full dynamics from large deviations & 9
\end{tabular}

$\begin{array}{llr}2.6 .2 & \text { Particle-hole excitations } & 10\end{array}$

$\begin{array}{lll}2.6 .3 & \mathrm{KdV} \text { solitons } & 12\end{array}$

$\begin{array}{lll}2.7 \text { Conclusions } & 14\end{array}$

$\begin{array}{lll}3 & \text { Riemann surfaces and ramified coverings } & \mathbf{1 5}\end{array}$

$\begin{array}{lll}3.1 & \text { Analytic continuation and Riemann surfaces } & 15\end{array}$

\begin{tabular}{lll}
\hline 3.2 & The Riemann surfaces $\mathcal{H}_{N}$ and $\mathcal{R}_{N}$ & 17
\end{tabular}

\begin{tabular}{lll}
\hline 3.3 Genus & 20
\end{tabular}

\begin{tabular}{lll}
\hline 3.4 & Ramified coverings & 21
\end{tabular}

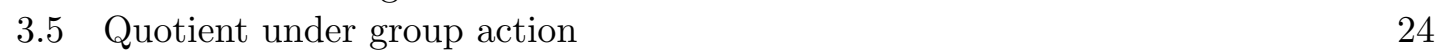

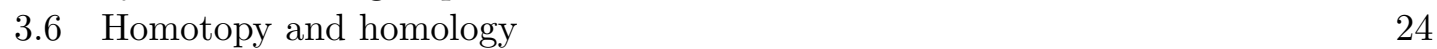

\begin{tabular}{|ll}
\hline 3.7 & Differential 1-forms \\
\hline 3.8 & 25
\end{tabular}

$\begin{array}{lll}3.8 & \text { Infinite genus limit } & 26\end{array}$

$\begin{array}{lll}3.8 .1 & \text { Riemann surface } \mathcal{R} & 26\end{array}$

\begin{tabular}{lll}
\hline 3.8 .2 & Riemann surface $\mathcal{R}$ & 28
\end{tabular}

\begin{tabular}{lll}
\hline 3.8 .3 & Riemann surface $\mathcal{R}^{\Delta}$ & 30
\end{tabular}

$\begin{array}{lll}3.8 .4 & \text { Collection } \overline{\mathcal{R}} \text { of Riemann surface } \mathcal{R}^{\Delta} & 31\end{array}$ 


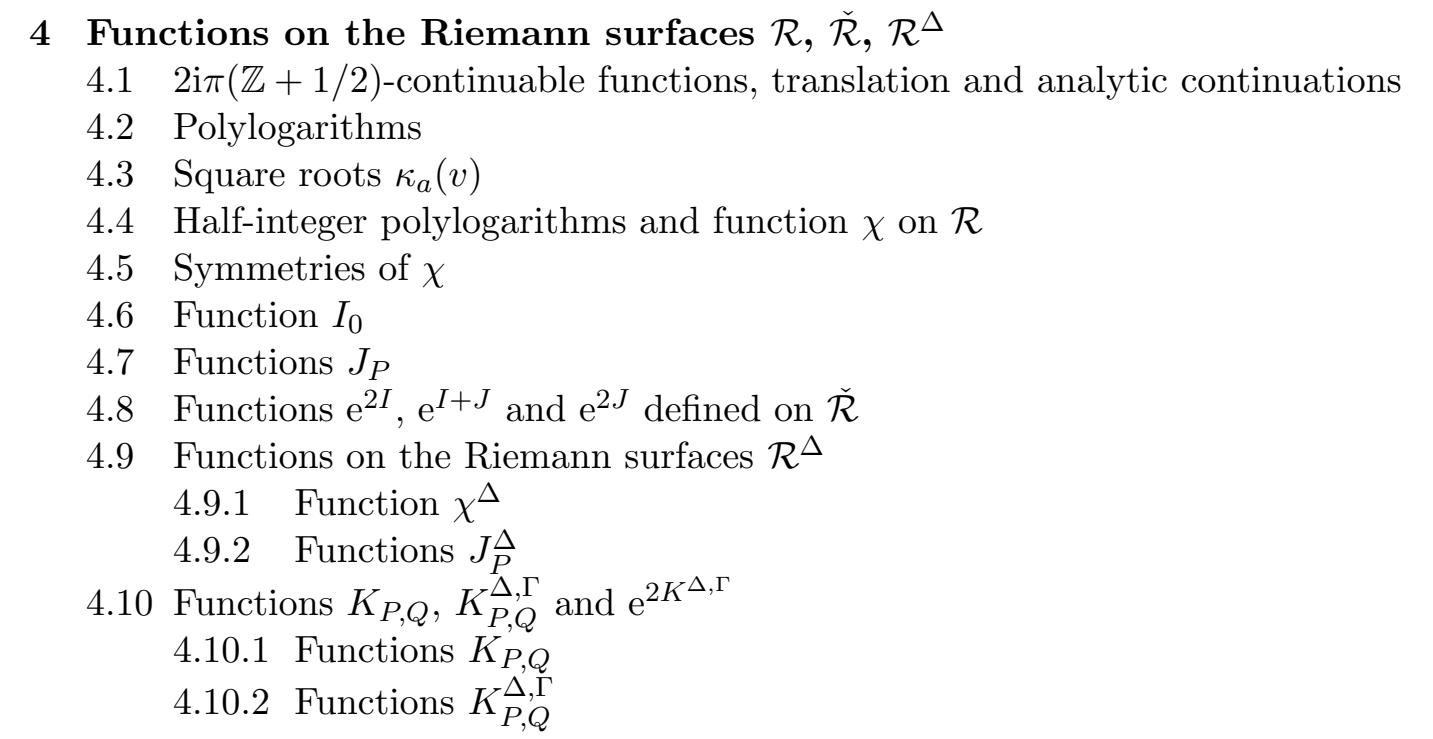

5 Relation with known formulas for KPZ

$\begin{array}{lll}5.1 & \text { Flat initial condition } & 52\end{array}$

$5.1 .1 \quad$ Relation with the generating function of the height 52

$\begin{array}{lll}5.1 .2 & \text { Relation with Fredholm determinants } & 53\end{array}$

\begin{tabular}{|ll}
5.2 & Sharp wedge initial condition \\
\hline
\end{tabular}

5.2.1 Relation with the generating function of the height from [39] $\quad 55$

5.2.2 Relation with the expression from Baik and Liu [40]] 56

\begin{tabular}{|lll}
5.3 & Multiple-time statistics with sharp wedge initial condition & 59
\end{tabular}

5.3.1 Derivation of (13) from Baik-Liu [42]] 59

\begin{tabular}{lll}
\hline 5.3 .2 & Pole structure of $(16)$ & 62
\end{tabular}

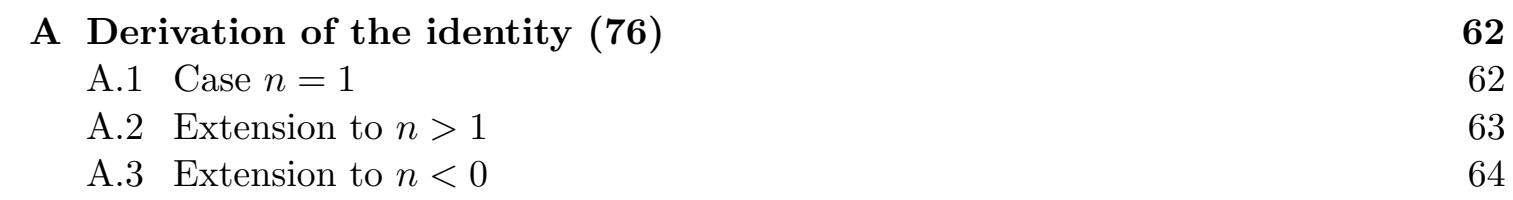

\begin{tabular}{|ll}
\hline B Derivation of the identities (106) and (107) & 64
\end{tabular}

B.1 Case $n=1$ for (106) 64

\begin{tabular}{|lll}
\hline B.2 & Extension to $n>1$ and $n<0$ & 65
\end{tabular}

\begin{tabular}{lll}
\hline B.3 Proof of (107) & 65
\end{tabular}

\begin{tabular}{|l|l} 
C Calculations of some integrals between $-\infty$ and $\nu \in \mathbb{D}$ & $\mathbf{6 6}$
\end{tabular}

C.1 The functions $\log \kappa_{a}$ and $\log \left(\kappa_{a}+\kappa_{b}\right)$ are analytic in $\mathbb{D} \quad 66$

$\begin{array}{lll}\text { C.2 Integral of } \kappa_{a}(v)^{-1} \kappa_{b}(v)^{-1} & 66\end{array}$

C.3 Integral of $\chi_{\emptyset}^{\prime \prime}(v) / \kappa_{a}(v)$ between $-\infty$ and a branch point $\quad 67$

C.4 Integral of $\chi_{\emptyset}^{\prime \prime}(v) / \kappa_{a}(v)$ as an infinite sum $\quad 67$

\begin{tabular}{|l|l|}
\hline D Calculations of some integrals depending on $(\nu, \mu) \in \mathbb{D}_{2}$ & $\mathbf{6 7}$
\end{tabular}

D.1 Domain of analyticity of functions $\log \left(\mathrm{e}^{\mathrm{i} \theta}\left(\kappa_{a}(\nu)+\kappa_{b}(\mu)\right)\right)$

$\begin{array}{lll}\text { D.2 Integral of } \kappa_{a}(u+\nu)^{-1} \kappa_{b}(u+\mu)^{-1} & 68\end{array}$

\begin{tabular}{|ll}
\hline D.3 Integral of $\kappa_{b}(u+\mu) / \kappa_{a}(u+\nu)$ & 68
\end{tabular}

\begin{tabular}{lll}
\hline D.4 Integral of $\chi_{\emptyset}^{\prime \prime}(u+\mu) / \kappa_{a}(u+\nu)$ & 69
\end{tabular}

\begin{tabular}{llc}
\hline E & Identities for the coefficients $W_{P}, W_{P}^{\Delta}$ & 69
\end{tabular}

$\begin{array}{llll}80 & \text { E.1 Differences of } W_{P} & 70\end{array}$ 
$\begin{array}{lll}\text { E.3 } & \text { Ratios of } \mathrm{e}^{2 W_{P}^{\Delta}} & 71\end{array}$

\section{Introduction}

KPZ universality in $1+1$ dimension [1 9$]$ describes large scale fluctuations appearing in a variety of systems such as growing interfaces [10], disordered conductors [11], onedimensional classical 12,14 and quantum 15 17 fluids, or traffic flow 18 . The height field $h_{\lambda}(x, t)$ characterizing KPZ universality depends on position $x \in \mathbb{R}$, time $t \geq 0$, and on a parameter $\lambda>0$ quantifying the strength of non-linear effects and the nonequilibrium character of the dynamics. The fluctuations of $h_{\lambda}(x, t)$ are believed to be universal in the sense that for a given geometry (infinite system, presence of various kinds of boundaries) and a given initial condition, the probability distribution of the appropriate height field $h_{\lambda}(x, t)$ is independent of the specific setting in KPZ universality and of the precise microscopic model studied at large scales. A prominent model, which has given its name to the universality class, is the KPZ equation [19], defined as the properly renormalized 2022 non-linear stochastic partial differential equation $\partial_{t} h_{\lambda}=\frac{1}{2} \partial_{x}^{2} h_{\lambda}-\lambda\left(\partial_{x} h_{\lambda}\right)^{2}+\eta$ with $\eta$ a unit space-time Gaussian white noise, and which is related by the Cole-Hopf transform $Z_{\lambda}(x, t)=\mathrm{e}^{-2 \lambda h_{\lambda}(x, t)}$ to the stochastic heat equation with multiplicative noise $\partial_{t} Z_{\lambda}=\frac{1}{2} \partial_{x}^{2} Z_{\lambda}-2 \lambda Z_{\lambda} \eta$ and Ito prescription in the time variable.

Of particular interest is the limiting object $h(x, t)=\lim _{\lambda \rightarrow \infty}\left(h_{\lambda}(x, t / \lambda)-\lambda^{2} t / 3\right)$ into the regime where non-linear effects dominate, and for which a number of exact results have been obtained in the past 20 years 2334 for the infinite system geometry $x \in \mathbb{R}$. Most notably, connections to random matrix theory have been identified: for given time $t \rightarrow \infty$ and position $x \in \mathbb{R}$, the probability distribution of $h(x, t)$ for specific initial conditions are equal to Tracy-Widom distributions [35], known for describing fluctuations of extremal eigenvalues in random matrix theory.

We are interested in this paper in KPZ universality in finite volume, specifically with periodic boundary conditions $x \equiv x+1$, in the strongly non-linear regime $\lambda \rightarrow \infty$. There, the standard deviation of $h(x, t)$ grows as $t^{1 / 3}$ at short times like in the infinite system $x \in \mathbb{R}$, before eventually saturating after the statistics of fluctuations has relaxed to a stationary distribution where $x \mapsto h(x, t)$ is Brownian. Large deviations in the stationary state away from typical Gaussian fluctuations are known explicitly [36, 37], and long time corrections to large deviations have been obtained explicitly [38] for a few specific initial conditions. Interestingly, the exact expressions in [36 38 involve polylogarithms with half-integer index. The analytical and topological structure of these special functions is at the heart of the present paper.

The complete evolution in time of KPZ fluctuations with periodic boundaries, crossing over between the short time limit, where the correlation length is much smaller than the system size and the fluctuations of the infinite system are recovered, and the long time limit where stationary large deviations appear, has been studied recently. Exact expressions have been obtained for the one-point 39 41] distribution $P(h(x, t)<u)$ of the height field for specific (sharp wedge, stationary and flat) initial conditions, as well as for the general, multiple-time joint distribution $P\left(h\left(x_{1}, t_{1}\right)<u_{1}, \ldots, h\left(x_{n}, t_{n}\right)<u_{n}\right)$ for sharp wedge initial condition [42]. All these exact expressions have a somewhat complicated 
structure involving combinations of square roots and half-integer polylogarithms.

A goal of the present paper is to show that the full crossover regime for KPZ fluctuations in finite volume have rather simple expressions using objects from algebraic geometry directly connected to stationary large deviations. More precisely, considering the (infinite genus) Riemann surface $\check{\mathcal{R}}$ on which polylogarithms with half-integer index are defined globally, the exact expression for the probability $\mathbb{P}(h(x, t)<u)$ with flat initial condition is rewritten as the integral around an infinitely long cylinder $\mathcal{C}$ of a holomorphic differential on $\check{\mathcal{R}}$ summed over all the sheets of a ramified covering from $\check{\mathcal{R}}$ to $\mathcal{C}$, see equation (1). Similar expressions are obtained for sharp wedge and stationary initial conditions, with an additional summation over finite subsets $\Delta$ of $\mathbb{Z}+1 / 2$, and $\check{\mathcal{R}}$ replaced by related Riemann surfaces $\mathcal{R}^{\Delta}$, see equations (6), (11). Multiple integrals around the cylinder as well as meromorphic functions on pairs of Riemann surfaces $\mathcal{R}^{\Delta} \times \mathcal{R}^{\Gamma}$ are additionally needed for the multiple-time joint distribution with sharp wedge initial condition, see equation (16).

The paper is organized as follows. In section 2, our main results expressing KPZ fluctuations with periodic boundaries in terms of infinite genus Riemann surfaces $\check{\mathcal{R}}, \mathcal{R}^{\Delta}$ and ramified coverings from them to the infinite cylinder $\mathcal{C}$ are given. Interpretations in terms of particle-hole excitations and $\mathrm{KdV}$ solitons are pointed out at the end of the section. In section 3, we recall some classical aspects of the theory of Riemann surfaces and ramified coverings used in the rest of the paper, and define the Riemann surfaces $\check{\mathcal{R}}$ and $\mathcal{R}^{\Delta}$. In section 4, we study several meromorphic functions defined on these Riemann surfaces and needed for KPZ fluctuations. Finally, we explain in section 5 how our main results from section 2 are related to earlier exact formulas 3942 . Some technical calculations are presented in appendix.

\section{KPZ fluctuations and Riemann surfaces}

In this section, we give exact expressions for KPZ fluctuations with periodic boundary conditions equivalent to those obtained in $39 \sqrt{42}$, but written in a more unified way by interpreting various terms as natural objects living on Riemann surfaces evaluated on distinct sheets. Connections to stationary large deviations and interpretations in terms of particle-hole excitations and $\mathrm{KdV}$ solitons are discussed in some detail toward the end of the section.

\section{$2.1 \quad$ Riemann surfaces $\check{\mathcal{R}}$ and $\mathcal{R}^{\Delta}$}

The exact formulas for KPZ fluctuations given below involve Riemann surfaces $\check{\mathcal{R}}$ and $\mathcal{R}^{\Delta}$, quotient under groups of holomorphic automorphisms of a Riemann surface $\mathcal{R}$ which has the topology of an infinite dimensional hypercube and which is a natural domain of definition for some infinite sums of square roots with branch points $2 \mathrm{i} \pi a, a \in \mathbb{Z}+1 / 2$. An introduction to several topics related to Riemann surfaces used in this paper is given in section 3, starting with a finite genus analogue $\mathcal{R}_{N}$ of $\mathcal{R}$ before giving precise definitions of $\check{\mathcal{R}}$ and $\mathcal{R}^{\Delta}$.

The Riemann surface $\mathcal{R}$ has a kind of translation invariance inherited from that of the branch points $2 \mathrm{i} \pi a$, and which is eliminated by definition in $\check{\mathcal{R}}$. The Riemann surfaces $\mathcal{R}^{\Delta}$ are indexed by finite sets $\Delta$ of half-integers, which we write as $\Delta \sqsubset \mathbb{Z}+1 / 2$. The elements $a \in \Delta$ index branch points $2 \mathrm{i} \pi a$ that have been removed from the infinite sum of square roots defined on $\mathcal{R}$. The translation invariance of $\mathcal{R}$ implies that $\mathcal{R}^{\Delta} \sim \mathcal{R}^{\Delta+1}$ are isomorphic Riemann surfaces. 


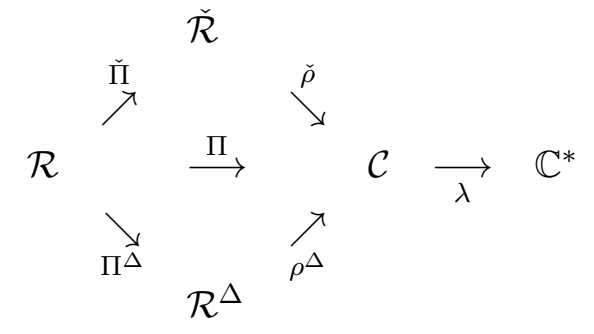

Figure 1: Summary of several useful covering maps between the Riemann surfaces considered in this paper.

The Riemann surface $\mathcal{R}$ can be partitioned into sheets $\mathbb{C}_{P}, P \sqsubset \mathbb{Z}+1 / 2$, copies of the complex plane glued together along branch cuts of the square roots, and we write $[v, P]$, $v \in \mathbb{C}, P \sqsubset \mathbb{Z}+1 / 2$ for a point of $\mathcal{R}$ with branch cuts chosen as in figure 18 right. The Riemann surfaces $\check{\mathcal{R}}$ and $\mathcal{R}^{\Delta}$ are identified as fundamental domains for corresponding group actions on $\mathcal{R}$, see respectively sections 3.8 .2 and 3.8 .3 , and may be partitioned by portions of the sheets $\mathbb{C}_{P}$. For $\check{\mathcal{R}}$, one can choose the union of infinite strips $\mathcal{S}_{P}^{0}=$ $\{[v, P],-\pi<\operatorname{Im} v \leq \pi\}, P \sqsubset \mathbb{Z}+1 / 2$, see figure 13 left. For $\mathcal{R}^{\Delta}$, a possible fundamental domain is the union of all $\mathbb{C}_{P}, P \cap \Delta=\emptyset$, see figure 16. Additionally, the collection of non-isomorphic $\mathcal{R}^{\Delta}, \Delta \equiv \Delta+1$, may also be partitioned into the infinite strips $\mathcal{S}_{P}^{0}$ from all $\mathcal{R}^{\Delta}$ without the restriction $\Delta \equiv \Delta+1$, see figure 17 .

The definitions of $\check{\mathcal{R}}$ and $\mathcal{R}^{\Delta}$ from $\mathcal{R}$ provide covering maps $\check{\Pi}$ and $\Pi^{\Delta}$ from $\mathcal{R}$ to $\check{\mathcal{R}}$ and $\mathcal{R}^{\Delta}$, see figure 1. Additionally, there exists natural covering maps $\check{\rho}$ and $\rho^{\Delta}$ from $\check{\mathcal{R}}$ and $\mathcal{R}^{\Delta}$ to the infinite cylinder $\mathcal{C}=\{v \in \mathbb{C}, v \equiv v+2 \mathrm{i} \pi\}$, with ramification points $[2 \mathrm{i} \pi a, P]$, $a \in \mathbb{Z}+1 / 2$ (and additionally $a \notin \Delta$ for $\rho^{\Delta}$ ). One-point statistics of the KPZ height field with periodic boundaries are expressed below for various initial conditions as an integral over a loop around the cylinder. The integrand involves holomorphic differentials traced over the covering maps $\check{\rho}$ or $\rho^{\Delta}$, i.e. summed over all the sheets of the Riemann surfaces covering the cylinder. The functions and meromorphic differentials needed are studied in detail in section 4 .

\section{$2.2 \quad$ Flat initial condition}

We consider in this section the one-point distribution $\mathbb{P}_{\text {flat }}(h(x, t)>u)$ of KPZ fluctuations with periodic boundary conditions, $h(x, t)=h(x+1, t)$, and flat initial condition $h(x, 0)=$ 0 . We claim that the properly renormalized random field $h(x, t)$ has the cumulative density function 1

$$
\mathbb{P}_{\text {flat }}(h(x, t)>u)=\int_{\gamma}\left(\operatorname{tr}_{\check{\rho}} Z_{t, u}^{\mathrm{flat}}\right)(\nu),
$$

with $\gamma$ a loop around the infinite cylinder $\mathcal{C}$ with winding number 1 . The holomorphic differential $Z_{t, u}^{\text {flat }}$ on the Riemann surface $\check{\mathcal{R}}$, defined away from ramification points of $\check{\rho}$ as

$$
Z_{t, u}^{\mathrm{flat}}([\nu, P])=\exp \left(\int_{[-\infty, \emptyset]}^{[\nu, P]} S_{t, u}^{\mathrm{fat}}\right) \frac{\mathrm{d} \nu}{2 \mathrm{i} \pi}
$$

\footnotetext{
${ }^{1}$ The definition of $h(x, t)$ used in this paper corresponds to a growth of the height function for TASEP in the positive direction, which after proper rescaling gives a growth of the KPZ height function in the negative direction, $h(x, t) \rightarrow-\infty$ when $t \rightarrow \infty$, corresponding to a negative coefficient $-\lambda \rightarrow-\infty$ in front of the non-linear term in the KPZ equation. The same convention for the sign of $u$ is used in 39]. The opposite convention is used in [40, with the notation $x=-u$ there.
} 
198

is built from an integral of the meromorphic differential $S_{t, u}^{\text {flat }}$ on $\check{\mathcal{R}}$ given by

$$
S_{t, u}^{\mathrm{flat}}(p)=\left(t \chi^{\prime}(p)-u \chi^{\prime \prime}(p)-\frac{1 / 4}{1+\mathrm{e}^{-v}}+\frac{\chi^{\prime \prime}(p)^{2}}{2}\right) \mathrm{d} v
$$

at $p=[v, P] \in \check{\mathcal{R}}$ away from ramification points of $\check{\rho}$. Here, $\chi, \chi^{\prime}, \chi^{\prime \prime}$ are meromorphic functions on $\check{\mathcal{R}}$, obtained by analytic continuations of the polylogarithm $\chi_{\emptyset}(\nu)=$ $-\mathrm{Li}_{5 / 2}\left(-\mathrm{e}^{\nu}\right) / \sqrt{2 \pi}$ and its derivatives, see sections 4.4 and 4.5 , and equations 66), 64, (57), (50) for precise definitions. We also refer to section 4 for explicit formulas for analytic continuations and proofs that $Z_{t, u}^{\text {flat }}$ is indeed holomorphic on $\check{\mathcal{R}}$ and independent from the path of integration in (2).

The trace of a meromorphic differential with respect to a covering map is defined in (34). For the covering map $\check{\rho}:[\nu, P] \mapsto \nu$ from the Riemann surface $\check{\mathcal{R}}$ to the infinite cylinder $\mathcal{C}$, the trace $\operatorname{tr}_{\check{\rho}}$ consists in summing over all the infinite strips $\mathcal{S}_{P}^{0}=\{[\nu, P],-\pi<$ $\operatorname{Im} \nu \leq \pi$ \} partitioning $\check{\mathcal{R}}$, see sections 3.8 .1 and 3.8 .2 for a precise definition of $\check{\mathcal{R}}$, and especially the left side of figure 13 for a graphical representation of how $\check{\mathcal{R}}$ is partitioned into infinite strips. The integral in $(1)$ is independent of the loop $\gamma$, $\operatorname{since}_{\operatorname{tr}_{\check{\rho}}} Z_{t, u}^{\text {flat }}$ is holomorphic on the cylinder $\mathcal{C}$ by the properties of the trace. With the change of variable $z=\mathrm{e}^{\nu}$, defining a covering map $\lambda$ from $\mathcal{C}$ to the punctured plane $\mathbb{C}^{*}=\mathbb{C} \backslash\{0\}$, the expression (1) can alternatively be written as the integral over a loop encircling 0 in $\mathbb{C}^{*}$, and $\mathbb{P}_{\text {flat }}(h(x, t)>u)$ is then the residue at the essential singularity $z=0$ of $\operatorname{tr}_{\lambda \circ \check{\rho}} Z_{t, u}^{\text {flat }}$.

The trace in (1) can be evaluated explicitly by considering a partition into infinite strips $\mathcal{S}_{P}^{0}, P \sqsubset \mathbb{Z}+1 / 2$ of the Riemann surface $\check{\mathcal{R}}$, see figure 13 left, so that $\left(\operatorname{tr}_{\check{\rho}} Z_{t, u}^{\text {flat }}\right)(\nu)=$ $\sum_{P \sqsubset \mathbb{Z}+1 / 2} Z_{t, u}^{\text {flat }}([\nu, P])$. In terms of the functions $\chi_{P}$ and $I_{0}$ defined in 64, , 68) one has

$$
\mathbb{P}_{\text {flat }}(h(x, t)>u)=\sum_{P \sqsubset \mathbb{Z}+1 / 2} \frac{(-1)^{|P|} V_{P}^{2}}{4^{|P|}} \int_{c-\mathrm{i} \pi}^{c+\mathrm{i} \pi} \frac{\mathrm{d} \nu}{2 \mathrm{i} \pi} \mathrm{e}^{t \chi_{P}(\nu)-u \chi_{P}^{\prime}(\nu)+I_{0}(\nu)+\frac{1}{2} f_{-\infty}^{\nu} \mathrm{d} v \chi_{P}^{\prime \prime}(v)^{2}},
$$

with $c \in \mathbb{R}^{*}$. The summation is over all finite subsets $P$ of $\mathbb{Z}+1 / 2$, and $V_{P}$ is the Vandermonde determinant

$$
V_{P}=\prod_{\substack{a, b \in P \\ a>b}}\left(\frac{2 \mathrm{i} \pi a}{4}-\frac{2 \mathrm{i} \pi b}{4}\right) .
$$

The function $\chi_{P}$ is the restriction of $\chi$ to the sheet $\mathbb{C}_{P}, I_{0}$ is given by $\mathrm{e}^{I_{0}(\nu)}=\left(1+\mathrm{e}^{\nu}\right)^{-1 / 4}$ if $c<0$ or $\mathrm{e}^{I_{0}(\nu)}=\mathrm{e}^{-\nu / 4}\left(1+\mathrm{e}^{-\nu}\right)^{-1 / 4}$ if $c>0$, and $f_{-\infty}^{\nu} \mathrm{d} v \chi_{P}^{\prime \prime}(v)^{2}=\lim _{\Lambda \rightarrow \infty}-|P|^{2} \log \Lambda+$ $\int_{-\Lambda}^{\nu} \mathrm{d} v \chi_{P}^{\prime \prime}(v)^{2}$. The extra factor $(-1)^{|P|} V_{P}^{2} / 4^{|P|}$ in 4 compared to 1 comes from the analytic continuation of $\int_{-\infty}^{\nu} \mathrm{d} v \chi_{\emptyset}^{\prime \prime}(v)^{2}$ from $\mathbb{C}_{\emptyset}$ to $\mathbb{C}_{P}$, see sections 4.7 and 4.8 .

The expression (1) is justified in section 5.1 by showing that (4) is equivalent to exact results obtained previously in [39, 40 from large scale asymptotics for the totally asymmetric simple exclusion process (TASEP), a discrete interface growth model in KPZ universality. This shows in particular that the corresponding expressions from [39] and 40] agree, which had not been properly derived before, and simply represent distinct choices for a fundamental domain $\check{\mathcal{R}}$ in $\mathcal{R}$.

The probability $\mathbb{P}_{\text {flat }}(h(x, t)>u)$ is interpreted in section 2.6.3 as a $N$-soliton $\tau$ function for the $\mathrm{KdV}$ equation, $N \rightarrow \infty$, averaged over the common velocity $\nu$ of the solitons, which is also identified as a moduli parameter for specific singular hyperelliptic Riemann surfaces. 


\subsection{Sharp wedge initial condition}

We consider in this section one-point statistics of KPZ fluctuations with sharp wedge ${ }^{2}$ initial condition $h(x, 0)=-\frac{|x-1 / 2|}{0^{+}}$, where $h(x, t)$ is defined after appropriate regularization. More generally, it is expected from large deviation results [38] that any initial condition of the form $h(x, 0)=h_{0}(x) / \epsilon$ where $h_{0}$ is continuous on the circle $x \equiv x+1$ with a global minimum 0 reached at $x=1 / 2$ only, is equivalent in the limit $\epsilon \rightarrow 0^{+}$to sharp wedge initial condition.

We show in section 5.2 that known exact formulas 39,40 for the cumulative density function of the KPZ height are equivalent to

$$
\mathbb{P}_{\mathrm{sw}}(h(x, t)>u)=\sum_{\substack{\Delta \sqsubset \mathbb{Z}+1 / 2 \\ \Delta \equiv \Delta+1}} \Xi_{x}^{\Delta} \int_{\gamma}\left(\operatorname{tr}_{\check{\rho}^{\Delta}} Z_{t, u}^{\Delta, \mathrm{sw}}\right)(\nu),
$$

with $\gamma$ as in $11, \check{\rho}^{\Delta}=\rho^{\Delta}, \Delta \neq \emptyset$ the covering map defined in section 3.8 .3 from $\mathcal{R}^{\Delta}$ to the infinite cylinder $\mathcal{C}$ and $\check{\rho}^{\emptyset}=\check{\rho}$ the covering map defined in section 3.8 .1 from $\check{\mathcal{R}}$ to $\mathcal{C}$, where $\check{\mathcal{R}}=\mathcal{R} / \check{\mathfrak{g}}$ is the quotient of $\mathcal{R}^{\emptyset}=\mathcal{R}$ by a group $\check{\mathfrak{g}}$ of translation automorphisms that exist only for $\Delta=\emptyset$. The trace with respect to $\check{\rho}^{\Delta}$ gives a holomorphic differential on the cylinder $\mathcal{C}$, periodic in $\nu$ with period $2 \mathrm{i} \pi$. The sum over non-isomorphic Riemann surfaces $\mathcal{R}^{\Delta}$ is weighted by

$$
\Xi_{x}^{\Delta}=(\mathrm{i} / 4)^{|\Delta|} \sum_{\substack{A \subset \Delta \\|A|=|\Delta \backslash A|}} \mathrm{e}^{2 \mathrm{i} \pi x\left(\sum_{a \in A} a-\sum_{a \in \Delta \backslash A} a\right)} V_{A}^{2} V_{\Delta \backslash A}^{2} .
$$

with $V_{A}$ the Vandermonde determinant (5) and $2 \pi\left(\sum_{a \in A} a-\sum_{a \in \Delta \backslash A} a\right)$ the momentum coupled to the coordinate $x$ along the interface, which appears only through $\Xi_{x}^{\Delta}$ in (6). Only sets $\Delta$ with cardinal $|\Delta|$ even contribute. The holomorphic differential

$$
Z_{t, u}^{\Delta, \mathrm{sw}}([\nu, P])=\exp \left(\int_{[-\infty, \emptyset]}^{[\nu, P]} S_{t, u}^{\Delta, \mathrm{sw}}\right) \frac{\mathrm{d} \nu}{2 \mathrm{i} \pi}
$$

is built from an integral with appropriate regularization at $[-\infty, \emptyset]$ of the meromorphic differential $S_{t, u}^{\Delta, \text { sw }}$ on $\mathcal{R}^{\Delta}$ given by

$$
S_{t, u}^{\Delta, \mathrm{sw}}(p)=\left(t \chi^{\prime \Delta}(p)-u \chi^{\prime \prime \Delta}(p)+\chi^{\prime \prime \Delta}(p)^{2}\right) \mathrm{d} v
$$

at $p=[v, P] \in \mathcal{R}^{\Delta}$ away from ramification points of $\rho^{\Delta}$. The functions $\chi^{\Delta}, \chi^{\prime \Delta}, \chi^{\prime \prime \Delta}$, analogues of $\chi, \chi^{\prime}, \chi^{\prime \prime}$ from the previous section with ramification points $[2 \mathrm{i} \pi a, P], a \in \Delta$ removed, are defined in (86), (91).

The trace in (6) can be evaluated more explicitly by considering appropriate partitions into sheets of the Riemann surfaces $\check{\mathcal{R}}$ and $\mathcal{R}^{\Delta}$. For the term $\Delta=\emptyset$, one has $\left(\operatorname{tr}_{\check{\rho}} Z_{t, u}^{\emptyset, \mathrm{sw}}\right)(\nu)=\sum_{P \sqsubset \mathbb{Z}+1 / 2} Z_{t, u}^{\emptyset, \mathrm{sw}}([\nu, P])$ like for flat initial condition, see figure 13 left. For $\Delta \neq \emptyset$, one has to sum instead over all strips $\mathcal{S}_{P}^{m}, P \cap \Delta=\emptyset, m \in \mathbb{Z}$, see figure 16 , leading to an integral between $c-\mathrm{i} \infty$ and $c+\mathrm{i} \infty$ of $Z_{t, u}^{\emptyset, \mathrm{sw}}([\nu, P])$, summed over all $P$, $P \cap \Delta=\emptyset$. The symmetry of the extension to $\overline{\mathcal{R}}$ of $Z_{t, u}^{\emptyset \text {,sw }}$ under the holomorphic automorphism $\overline{\mathcal{T}}$ defined in 44 , see section 3.8.4, allows to sum instead over all $\Delta$ and not just equivalence classes $\Delta \equiv \Delta+1$, and integrate only over the strip $\mathcal{S}_{P}^{0}$ from each $\mathcal{R}^{\Delta}$. Using

\footnotetext{
${ }^{2}$ Also called step or domain wall initial condition in the context of TASEP as a microscopic model.
} 
explicit analytic continuations from section 4.9 .2 , we finally obtain that $(6)$ is equivalent to the more explicit expression

$$
\begin{aligned}
\mathbb{P}_{\mathrm{sW}}(h(x, t)>u)=\sum_{\Delta \sqsubset \mathbb{Z}+1 / 2} \Xi_{x}^{\Delta} & \sum_{\substack{P \subset \mathbb{Z}+1 / 2 \\
P \cap \Delta=\emptyset}}(\mathrm{i} / 4)^{2|P|}\left(\prod_{a \in P} \prod_{\substack{b \in P \cup \Delta \\
b \neq a}}\left(\frac{2 \mathrm{i} \pi a}{4}-\frac{2 \mathrm{i} \pi b}{4}\right)^{2}\right) \\
& \times \int_{c-\mathrm{i} \pi}^{c+\mathrm{i} \pi} \frac{\mathrm{d} \nu}{2 \mathrm{i} \pi} \mathrm{e}^{t \chi_{P}^{\Delta}(\nu)-u \chi_{P}^{\prime \Delta}(\nu)+f_{-\infty}^{\nu} \mathrm{d} v \chi_{P}^{\prime \prime \Delta}(v)^{2}},
\end{aligned}
$$

with $|P|$ the number of elements in $P, \chi_{P}^{\Delta}$ the restriction of $\chi^{\Delta}$ to the sheet $\mathbb{C}_{P}$ of $\mathcal{R}^{\Delta}$ given in (87), and $f$ the regularized integral subtracting the divergent logarithmic term at $-\infty$ like in $(92)$. The expression (10) is derived in section 5.2 from earlier works [39] and 40 using the structure of the Riemann surfaces $\mathcal{R}^{\Delta}$ detailed in section 3.8.3 and explicit analytic continuations obtained in section 4.9.2. This shows in particular that the expressions from [39] and [40] about sharp wedge initial condition agree, which was missing so far.

\subsection{Stationary initial condition}

Exact results have also been obtained for one-point statistics of the KPZ height with stationary initial condition [39, 41, where $x \mapsto h(x, 0)$ is a standard Brownian bridge. The formulas in that case are essentially the same as for sharp wedge initial condition, with only an additional harmless factor. Starting either with equation (7) of [39] (for $x=0$ ) or with equation (2.1) of [41] for general $x$, we obtain by comparison to (6)

$$
\mathbb{P}_{\text {stat }}(h(x, t)>u)=\sum_{\substack{\Delta \sqsubset \mathbb{Z}+1 / 2 \\ \Delta \equiv \Delta+1}} \Xi_{x}^{\Delta} \int_{\gamma}\left(\operatorname{tr}_{\check{\rho} \Delta} Z_{t, u}^{\Delta, \text { stat }}\right)(\nu),
$$

with

$$
Z_{t, u}^{\Delta, \text { stat }}([\nu, P])=-\sqrt{2 \pi} \mathrm{e}^{-\nu} \partial_{u} Z_{t, u}^{\Delta, \mathrm{sw}}([\nu, P])
$$

and the same notations as in (6). A more explicit formula can be written by inserting the extra factor $-\sqrt{2 \pi} \mathrm{e}^{-\nu} \partial_{u}$ into (10).

\subsection{Multiple-time statistics with sharp wedge initial condition}

The joint distribution of the height at multiple times $0<t_{1}<\ldots<t_{n}$ and corresponding positions $x_{j}$ was obtained by Baik and Liu for sharp wedge initial condition in [42]. After some rewriting in section 5.3 .1 based on explicit analytic continuations from section 4.9 and 4.10 , we obtain

$$
\begin{aligned}
& \mathbb{P}\left(h\left(x_{1}, t_{1}\right)>u_{1}, \ldots, h\left(x_{n}, t_{n}\right)>u_{n}\right) \\
& =\left(\prod_{\ell=1}^{n} \sum_{\Delta_{\ell} \sqsubset \mathbb{Z}+1 / 2} \sum_{\substack{P_{\ell} \sqsubset \mathbb{Z}+1 / 2 \\
P_{\ell} \cap \Delta_{\ell}=\emptyset}}\right) \int_{c_{1}-\mathrm{i} \pi}^{c_{1}+\mathrm{i} \pi} \frac{\mathrm{d} \nu_{1}}{2 \mathrm{i} \pi} \ldots \int_{c_{n}-\mathrm{i} \pi}^{c_{n}+\mathrm{i} \pi} \frac{\mathrm{d} \nu_{n}}{2 \mathrm{i} \pi} \Xi_{x_{1}, \ldots, x_{n}}^{\Delta_{1}, \ldots, \Delta_{n}}\left(\nu_{1}, \ldots, \nu_{n}\right)
\end{aligned}
$$

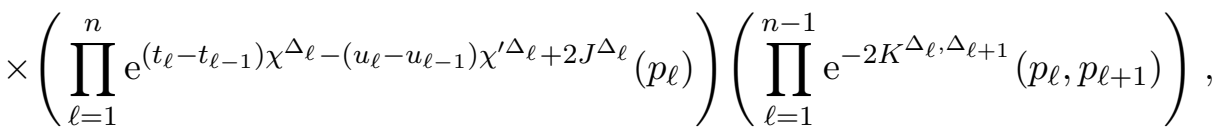

with $t_{0}=u_{0}=0, c_{n}<\ldots<c_{1}<0, p_{\ell}=\left[\nu_{\ell}, P_{\ell}\right]$ a point on the Riemann surface $\mathcal{R}^{\Delta_{\ell}}, \chi^{\Delta}$ and $\chi^{\prime \Delta}$ holomorphic functions on $\mathcal{R}^{\Delta}$ given in $86,, 91$, $\mathrm{e}^{2 J^{\Delta}}$ the meromorphic function 
on $\mathcal{R}^{\Delta}$ from 96 and $\mathrm{e}^{2 K^{\Delta, \Gamma}}$ a meromorphic function on $\mathcal{R}^{\Delta} \times \mathcal{R}^{\Gamma}$ defined by 118 . The collection of Riemann surfaces $\mathcal{R}^{\Delta_{\ell}}$ in $\sqrt{13}$ is weighted by the meromorphic function on $\mathbb{C}^{n}$

$$
\begin{aligned}
& \Xi_{x_{1}, \ldots, x_{n}}^{\Delta_{1}, \ldots, \Delta_{n}}\left(\nu_{1}, \ldots, \nu_{n}\right) \\
& =\left(\prod_{\ell=1}^{n} \sum_{\substack{A_{\ell} \sqsubset \Delta_{\ell} \\
\left|A_{\ell}\right|=\left|\Delta_{\ell} \backslash A_{\ell}\right|}}\right) \prod_{\ell=1}^{n}\left((\mathrm{i} / 4)^{\left|\Delta_{\ell}\right|} V_{A_{\ell}}^{2} V_{\Delta_{\ell} \backslash A_{\ell}}^{2} \mathrm{e}^{2 \mathrm{i} \pi\left(x_{\ell}-x_{\ell-1}\right)\left(\sum_{a \in A_{\ell}} a-\sum_{a \in \Delta_{\ell} \backslash A_{\ell}} a\right)}\right) \\
& \times \prod_{\ell=1}^{n-1} \frac{\left(1-\mathrm{e}^{\nu_{\ell+1}-\nu_{\ell}}\right)^{\left|\Delta_{\ell}\right| / 2}\left(1-\mathrm{e}^{\nu_{\ell}-\nu_{\ell+1}}\right)^{\left|\Delta_{\ell+1}\right| / 2}}{\left(1-\mathrm{e}^{\nu_{\ell+1}-\nu_{\ell}}\right) V_{A_{\ell}, A_{\ell+1}}\left(\nu_{\ell}, \nu_{\ell+1}\right) V_{\Delta_{\ell} \backslash A_{\ell}, \Delta_{\ell+1} \backslash A_{\ell+1}}\left(\nu_{\ell}, \nu_{\ell+1}\right)},
\end{aligned}
$$

with $x_{0}=0, V_{A}$ the Vandermonde determinant (5) and

$$
V_{A, B}(\nu, \mu)=\prod_{a \in A} \prod_{b \in B}\left(\frac{2 \mathrm{i} \pi a-\nu}{4}-\frac{2 \mathrm{i} \pi b-\mu}{4}\right) .
$$

Since $\Xi_{x_{1}, \ldots, x_{n}}^{\Delta_{1}, \ldots, \Delta_{n}}\left(\nu_{1}, \ldots, \nu_{n}\right)=0$ when any of the $\left|\Delta_{\ell}\right|$ is odd because of the constraints $\left|A_{\ell}\right|=\left|\Delta_{\ell} \backslash A_{\ell}\right|$, only sets $\Delta_{\ell}$ containing an even number of elements contribute to (13).

The same reasoning as the one between (10) and (6) allows to express (13) in terms of non-isomorphic Riemann surfaces and a trace over covering maps. One has

$$
\begin{aligned}
& \mathbb{P}\left(h\left(x_{1}, t_{1}\right)>u_{1}, \ldots, h\left(x_{n}, t_{n}\right)>u_{n}\right) \\
& =\left(\prod_{\ell=1}^{n} \sum_{\substack{\Delta_{\ell} \sqsubset \mathbb{Z}+1 / 2 \\
\Delta_{\ell} \equiv \Delta_{\ell}+1}}\right) \int_{\gamma_{1}} \ldots \int_{\gamma_{n}} \Xi_{x_{1}, \ldots, x_{n}}^{\Delta_{1}, \ldots, \Delta_{n}}\left(\nu_{1}, \ldots, \nu_{n}\right) \\
& \quad \times \operatorname{tr}_{\check{\rho}^{\Delta_{1}}} \ldots \operatorname{tr}_{\check{\rho} \Delta_{n}} \frac{\prod_{\ell=1}^{n} Z_{t_{\ell}-t_{\ell-1}, u_{\ell}-u_{\ell-1}}^{\Delta_{\ell}, \mathrm{sw}}\left(\nu_{\ell}\right)}{\prod_{\ell=1}^{n-1} \mathrm{e}^{2 K^{\Delta_{\ell}, \Delta_{\ell+1}}\left(\nu_{\ell}, \nu_{\ell+1}\right)},}
\end{aligned}
$$

with $\operatorname{tr}_{\check{\rho}^{\Delta}}$ acting on $\nu_{\ell}, Z_{\delta t, \delta u}^{\Delta \text {,sw }}$ the holomorphic differential from (8), and a loop $\gamma_{\ell}$ with winding number 1 around the infinite cylinder $\mathcal{C}$ for the variable $\nu_{\ell}$. Because of the trace, the integrand in (16) is meromorphic in $\mathcal{C}^{n}$. The loops $\gamma_{\ell}$ do not cross each other, as the order $\operatorname{Re} \nu_{n}<\ldots<\operatorname{Re} \nu_{1}$ must be preserved because of the presence of simple poles at $\nu_{\ell+1}=\nu_{\ell}+2 \mathrm{i} \pi m, m \in \mathbb{Z}$. Interestingly, it can be shown that such poles exist only when $\Delta_{\ell+1}=\Delta_{\ell}+m$ and $P_{\ell+1}=P_{\ell}+m$ (and only the sector $A_{\ell+1}=A_{\ell}+m$ of $\Xi_{x_{1}, \ldots, x_{n}}^{\Delta_{1}, \ldots, \Delta_{n}}$ contributes to them), corresponding to points $p_{\ell}=\left[\nu_{\ell}, P_{\ell}\right]$ and $p_{\ell+1}=\left[\nu_{\ell+1}, P_{\ell+1}\right]$ coinciding on the Riemann surface $\mathcal{R}^{\Delta_{\ell}} \sim \mathcal{R}^{\Delta_{\ell+1}}$, see section 5.3.2.

\subsection{Discussion}

In this section, we discuss various interpretations of the exact formulas given above for KPZ fluctuations with periodic boundaries.

\subsubsection{Full dynamics from large deviations}

In the long time limit, KPZ fluctuations in finite volume converge to a stationary state where the interface has the same statistics as a Brownian motion with appropriate boundary conditions. Large deviations corresponding to fluctuations of the height with an amplitude of order $t$ when $t \rightarrow \infty$ are on the other hand non-Gaussian and can be characterized by a generating function of the form 36,43

$$
\left\langle\mathrm{e}^{s h(x, t)}\right\rangle \simeq \theta(s) \mathrm{e}^{t e(s)},
$$


where $e(s)$ involves an infinite sum of square roots. At finite time, the generating function of the height is given exactly by a sum of infinitely many terms of the same form,

$$
\left\langle\mathrm{e}^{s h(x, t)}\right\rangle=\sum_{n} \theta_{n}(s) \mathrm{e}^{t e_{n}(s)}
$$

with $n$ an index labelling sheets of Riemann surfaces, see equations (122), (131). Known results for the spectrum of TASEP 44,45$]$ indicate that the stationary contribution corresponds to the sheet $\mathbb{C}_{\emptyset}$ of $\mathcal{R}$ when $\operatorname{Re} s>0$.

These observations suggest the possibility to guess the full finite time dynamics of KPZ fluctuations in finite volume from the solution of the static problem of stationary large deviations alone. For flat initial condition in particular, the functions $\theta_{P}(s), e_{P}(s)$ (or, more properly, their analogues for the probability (4) after Fourier transform, see section 5.1.1) are simply analytic continuations of $\theta_{\emptyset}(s), e_{\emptyset}(s)$ to all the sheets of the Riemann surface $\check{\mathcal{R}}$. The situation is less straightforward for sharp wedge and stationary initial conditions, where a natural interpretation is still missing for the coefficients $\Xi_{x}^{\Delta}$ weighting the Riemann surfaces $\mathcal{R}^{\Delta}$ covered by $\mathcal{R}$ in (6) and (11).

The stationary large deviations problem can be studied independently from the dynamics, using e.g. matrix product representations for discrete models [46, 47]. This approach was recently exploited in 38 to express the factor $\theta(s)$ in 17$)$ for general initial condition as the probability that a gas of infinitely many non-intersecting Brownian bridges with density $1 / s$ stays under the graph of the initial condition $h(x, 0)=h_{0}(x)$. More precisely, it was shown that

$$
\theta(s)=\frac{\mathbb{P}\left(b_{-1}<h_{0} \mid \ldots<b_{-2}<b_{-1}\right)}{\mathbb{P}\left(b_{-1}<b_{0} \mid \ldots<b_{-2}<b_{-1}, b_{1}<b_{2}<\ldots\right)},
$$

where $b_{j}(x)-b_{j}(0), j \in \mathbb{Z}$ are independent standard Brownian bridges with $b_{j}(0)=b_{j}(1)$, distances between consecutive endpoints $b_{j+1}(0)-b_{j}(0)$ are independent exponentially distributed random variables with parameter $s$, and $b_{0}(0)=h_{0}(0)$. Deriving exact formulas from the Brownian bridge representation is still an open problem, though, even for the simple initial conditions (flat, Brownian, sharp wedge) for which the result is known from Bethe ansatz, see however [48] for related work.

The idea that the contributions of the excited states of a theory should follow from that of the ground state by analytic continuation with respect to some parameter is not new, see for instance [49] for the quantum quartic oscillator, [50] for the Ising field theory on a circle (where the ground state energy is interestingly also given by an infinite sum of square roots, but with conjugate branch points paired), or [51] for models described by the thermodynamic Bethe ansatz. In the context of the Schrödinger equation for a particle in a potential, a unifying scheme appears to be exact WKB analysis [52], which uses tools from the theory of resurgent functions in order to reconstruct a single valued eigenfunction from the multivalued classical action. Such an approach might be useful for $\mathrm{KPZ}$ in order to derive known exact formulas without having to consider discrete models, by starting directly from the associated backward Fokker-Planck equation, a rather formal infinite dimensional linear partial differential equation acting on the functional space of allowed initial heights.

\subsubsection{Particle-hole excitations}

The finite sets of half-integers labelling the sheets of the Riemann surfaces $\check{\mathcal{R}}, \mathcal{R}^{\Delta}$ considered in this paper have a natural interpretation in terms of particle-hole excitations 

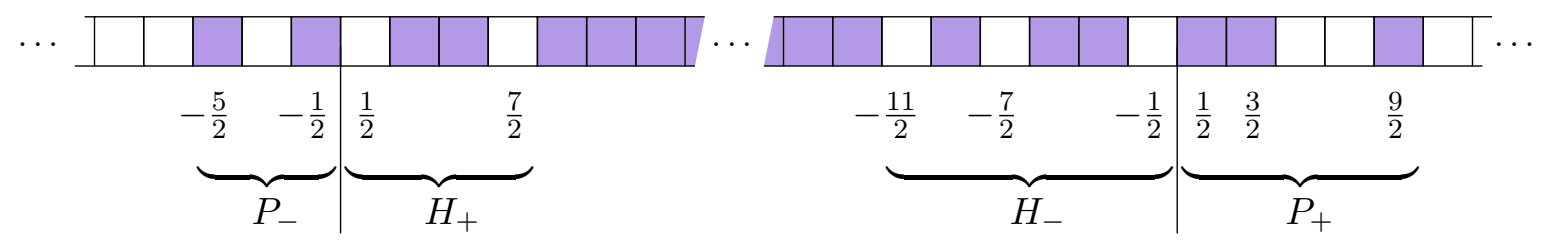

Figure 2: Picture of particle-hole excitations at both edges of the Fermi sea corresponding to sets $P, H$ of half-integers. The notations $P_{ \pm}, H_{ \pm}$indicate the positive and negative elements of the sets.

at both edges of a Fermi sea, see figure 2. This is most clearly seen on the expressions from [39] for the generating function $\left\langle\mathrm{e}^{s h(x, t)}\right\rangle$ of the KPZ height discussed in sections 5.1.1 and 5.2 .1 .

From the exact Bethe ansatz solution of TASEP, eigenstates of the time evolution operator in the KPZ scaling regime are labelled by sets $P$ and $H$ corresponding to particlehole excitations, interpreted respectively as momenta of quasiparticle and hole excitations relative to the Fermi momentum on both sides of the Fermi sea, see figure 2. Excitations only occur in particle-hole pairs, with "neutral charge": no particle or hole excitation alone occurs. The stationary state $P=H=\emptyset$ corresponds in particular to the completely filled Fermi sea.

For sharp wedge initial condition, the sets $P$ and $H$ must satisfy the constraint $|P|_{ \pm}=$ $|H|_{\mp}$ that the number of positive elements of $P$ is equal to the number of negative elements of $H$ and vice versa, see equations (131), (133). This corresponds to the fact that particlehole excitations occur independently on both sides of the Fermi sea, i.e. quasiparticles at a finite distance from either side of the Fermi sea may be excited above the Fermi momentum but will stay at a finite distance of the same edge of the Fermi sea: excitation from one edge to the other (known as Umklapp processes in condensed matter physics) are suppressed for KPZ. It is remarkable that the constraints $|P|_{ \pm}=|H|_{\mp}$ are automatically verified in (6), 11) from the way the collection of non-isomorphic Riemann surfaces $\mathcal{R}^{\Delta}$ are partitioned into sheets $\mathbb{C}_{P}$, with $H=P \ominus \Delta$ the symmetric difference of $P$ and $\Delta$ (union minus intersection), see section 5.2.1.

Flat initial condition 122 , 123 corresponds to the special case $P=H$, where momenta of quasiparticles excitations on one side of the Fermi sea are identical to momenta of hole excitations on the other side of the Fermi sea. It is again quite remarkable that the resulting constraint $|P|_{+}=|P|_{-}$naturally appears for the sets $P$ labelling (half)-sheets of the Riemann surface $\check{\mathcal{R}}$, see figure 13 on the right. The extra constraint $P=H$ for flat initial condition is understood from TASEP as the fact that a specific microscopic state representing a flat interface has nonzero overlap only with Bethe eigenstates corresponding to particle-hole excitations satisfying the constraint [53]. This has the consequence to increase the spectral gap (i.e. to reduce the relaxation time) compared to a generic initial state, as was already recognized in [54].

Compared to the states contributing for flat initial condition, which have zero momentum, a non-empty symmetric difference $\Delta=P \ominus H$ corresponds to an imbalance between both sides of the Fermi sea, and is related through the coefficients $\Xi_{x}^{\Delta}$ in (7) to motion along the KPZ interface, with momentum $2 \pi\left(\sum_{a \in P} a-\sum_{a \in H} a\right)$.

The interpretation of KPZ fluctuations in terms of particle-hole excitations close to the Fermi level is reminiscent of Luttinger liquid universality describing large scale dynamics of one-dimensional quantum fluids, with however several important distinctions. In addition to the absence mentioned above of Umklapp terms for KPZ, unlike in the Luttinger liquid setting, the dispersion relation, linear for the Luttinger liquid by construction after 
with the $\tau$ function defined by

$$
\tau_{\text {flat }}(x, t ; \nu)=\mathrm{e}^{3 t \chi_{\emptyset}(\nu)-x \chi_{\emptyset}^{\prime}(\nu)+I_{0}(\nu)+J_{\emptyset}(\nu)} \operatorname{det}\left(1-M_{\text {flat }}(x, t ; \nu)\right),
$$

\footnotetext{
${ }^{3}$ The change $(t, u, x) \rightarrow(3 t, x, y)$ in this section is needed to conform to standard notations for KdV.

${ }^{4}$ The exponential of a linear function of $x$ in front of the Fredholm determinant does not contribute to $u_{\text {flat }}=2 \partial_{x}^{2} \log \tau_{\text {flat }}$, which is thus still solution of the KdV equation.
} 
where $\chi_{\emptyset}$ is defined in (57), $I_{0}$ in (68) and $J_{\emptyset}$ in (74). The operator $M_{\text {flat }}(x, t ; \nu)$, acting on sequences indexed by $\mathbb{Z}+1 / 2$, has the kernel

$$
M_{\mathrm{flat}}(x, t ; \nu)_{a, b}=\frac{\mathrm{e}^{2 x \kappa_{a}(\nu)+2 t \kappa_{a}^{3}(\nu)+2 \int_{-\infty}^{\nu} \mathrm{d} v \frac{\chi_{\emptyset}^{\prime \prime}(v)}{\kappa_{a}(v)}}}{\kappa_{a}(\nu)\left(\kappa_{a}(\nu)+\kappa_{b}(\nu)\right)}
$$

with $\kappa_{a}(v)$ a specific branch of $\sqrt{4 \mathrm{i} \pi a-2 v}$ defined in $(50)$. When $c<0$, this is directly the result from [40], see equations (124), (125). When $c>0$, a little more work is needed in order to rewrite into (22) the integral for $\nu$ between $c-\mathrm{i} \infty$ and $c+\mathrm{i} \infty$ of the Fredholm determinant in 39, which corresponds to a representation of $\check{\mathcal{R}}$ distinct from the one in (4), see section 5.1.1.

KPZ with flat initial condition thus involves a $\tau$ function for $\mathrm{KdV}$, i.e. $u_{\text {flat }}(x, t ; \nu)=$ $2 \partial_{x}^{2} \log \tau_{\text {flat }}(x, t ; \nu)$ is a solution of the KdV equation 20 for any $\nu$. This solution corresponds to a gas of infinitely many solitons with (complex) velocities $-\kappa_{a}^{2}(v)=2 \nu-4 \mathrm{i} \pi a$, $a \in \mathbb{Z}+1 / 2$. The probability $\mathbb{P}_{\text {flat }}(h(y, 3 t)>x)$ is obtained by averaging $\tau_{\text {flat }}(x, t ; \nu)$ over the common velocity modulo $2 \mathrm{i} \pi$ of the solitons. Time variables for higher equations in the KdV hierarchy naturally appear in 24 as $t_{2 m+1}=\chi_{\emptyset}^{(m+2)}(\nu) /(2 m+1) !$ !, see equation (187).

The possibility to interpret KPZ as a gas of solitons was put forward by Fogedby [58 starting with the WKB solution of the Fokker-Planck equation in the weak noise limit, with in particular the prediction of the dispersion relation $|k|^{3 / 2}$ as a function of momentum $k$, corresponding in our notations to $\kappa_{a}^{3}(\nu) \sim|a|^{3 / 2}$ for large $a$, see also $[59$ for recent related work.

Since flat initial condition corresponds to $h(y, 0)=0$, the probability $\mathbb{P}_{\text {flat }}(h(y, 3 t)>x)$ is expected to converge to $1_{\{x<0\}}$ when $t \rightarrow 0$. Furthermore, since the KPZ height in finite volume at short time must have the same statistics as the KPZ fixed point on $\mathbb{R}[60$, one should have $\mathbb{P}_{\text {flat }}\left(h(y, 4 t)>-t^{1 / 3} x\right) \rightarrow F_{1}(x)$ when $t \rightarrow 0$, where $F_{1}$ is the GOE TracyWidom distribution from random matrix theory. This was checked numerically with good agreement in 39. In terms of the $\mathrm{KdV}$ interpretation, we conjecture that the short time limit corresponds to the known scaling solution $(t / 4)^{2 / 3} u\left(-(t / 4)^{1 / 3} x, t / 3\right)=V^{\prime}(x)-V^{2}(x)$ of $20 p$, where $V$ is a solution of the Painlevé II equation $V^{\prime \prime}(z)=2 V^{3}(z)+z V(z)+\alpha$ and $\alpha$ a constant which may depend on $\nu$, see e.g. 61].

The relation to $\mathrm{KdV}$ allows to interpret the integration variable $\nu$ in (1) as a moduli parameter for a class of singular hyperelliptic Riemann surfaces with infinitely many branch points. A soliton solutions of $\mathrm{KdV}$ can indeed be seen as the limit $\delta \rightarrow 0$ of a solution of $\mathrm{KdV}$ built in terms of the theta function of the hyperelliptic Riemann surface with branch points $0, \infty, \kappa_{a}^{2}+\delta, \kappa_{a}^{2}-\delta$ when branch points $\kappa_{a}^{2} \pm \delta$ merge together on the Riemann surface, see [55, 56]. The $\infty$-soliton solution $u_{\text {flat }}$ corresponds in particular to the hyperelliptic Riemann surface with branch points $0, \infty$ and singular points $4 \mathrm{i} \pi a-2 \nu$, $a \in \mathbb{Z}+1 / 2$, or equivalently to branch points $\nu, \infty$ and singular points $2 \mathrm{i} \pi a, a \in \mathbb{Z}+1 / 2$. The Riemann surface $\mathcal{R}$ on which the parameter $\nu$ lives before taking the trace in (1) thus describes the monodromy of the branch point $\nu$ of the hyperelliptic Riemann surface above around the singular points $2 \mathrm{i} \pi a$.

For sharp wedge initial condition, the Fredholm determinant expressions for $\mathbb{P}_{\mathrm{sw}}(h(2 y$, $3 t)>x$ ) from [39, 40] are instead reminiscent of soliton solutions for the KadomtsevPetviashvili (KP) equation $3 \partial_{y}^{2} u=\partial_{x}\left(4 \partial_{t} u-\left(6 u \partial_{x} u+\partial_{x}^{3} u\right)\right)$, a generalization of the $\mathrm{KdV}$ equation for a function $u(x, y, t)$ with two spatial dimensions, see e.g. [55 57]. The $\tau$ functions related to $u$ by $u(x, y, t)=2 \partial_{x}^{2} \log \tau(x, y, t)$ and corresponding to $N$-soliton solutions of the KP equation are of the form $\tau(x, y, t)=\operatorname{det}\left(1-M_{N}^{\mathrm{KP}}(x, y, t)\right)$, where the $N \times N$ matrix $M_{N}^{\mathrm{KP}}(x, y, t)_{a, b}=\mathrm{e}^{x\left(\kappa_{a}-\eta_{b}\right)+y\left(\kappa_{a}^{2}-\eta_{b}^{2}\right)+t\left(\kappa_{a}^{3}-\eta_{b}^{3}\right)+\lambda_{a}} /\left(\kappa_{a}-\eta_{b}\right)$ depends on $3 N$ 
arbitrary constants $\kappa_{a}, \eta_{b}, \lambda_{a}$. For KPZ with sharp wedge initial condition, calculations similar to the ones leading to $(22)$, see section 5.2 .2 for more details, allow to rewrite $(10)$ in terms of a Fredholm determinant as

$$
\mathbb{P}_{\mathrm{SW}}(h(2 y, 3 t)>x)=\int_{c-\mathrm{i} \pi}^{c+\mathrm{i} \pi} \frac{\mathrm{d} \nu}{2 \mathrm{i} \pi} \mathrm{e}^{t \chi_{\emptyset}(\nu)-u \chi_{\emptyset}^{\prime}(\nu)+2 J_{\emptyset}(\nu)} \operatorname{det}\left(1-M_{\mathrm{SW}}(x, y, t ; \nu)\right),
$$

with $M_{\mathrm{sw}}(x, y, t ; \nu)=L_{\mathrm{sw}}(x, y, t ; \nu) L_{\mathrm{sw}}(x,-y, t ; \nu)$ and

$$
L_{\mathrm{sw}}(x, y, t ; \nu)_{a, b}=\frac{\mathrm{e}^{x \kappa_{a}(\nu)+y \kappa_{a}^{2}(\nu)+t \kappa_{a}^{3}(\nu)+2 \int_{-\infty}^{\nu} \mathrm{d} v \frac{\chi_{\emptyset}^{\prime \prime}(v)}{\kappa_{a}(v)}}}{\kappa_{a}(\nu)\left(\kappa_{a}(\nu)+\kappa_{b}(\nu)\right)} .
$$

When $c<0$, this is essentially the result from 40, see section 5.2.2. A similar Fredholm determinant was also given in 39 for $c>0$, corresponding to a distinct representation of the Riemann surfaces in $(10)$.

The dependency on $x, y$ and $t$ in (25) is essentially the same as for KP solitons, with $N \rightarrow \infty, \kappa_{a}=\kappa_{a}(\nu)$ and $\eta_{b}=-\kappa_{b}(\nu)$. The rest of the expression is similar to but different from the Cauchy determinant $M_{N}^{\mathrm{KP}}$ required for KP solitons. Interestingly, proper $\infty$-soliton solutions of the KP hierarchy are known to appear for Laplacian growth 62, which belongs to a universality class of growing interfaces distinct from KPZ.

A paper by Quastel and Remenik [63] about KPZ fluctuations on $\mathbb{R}$ appeared shortly after our paper. There, the one-point cumulative distribution function with general initial condition is shown to be a $\tau$ function for the KP equation, without an extra integration like in (22), while multiple-point distributions at a given time correspond to a matrix generalization of KP. This suggest that there might still be a way to properly understand (25) in terms of KP solitons, maybe a matrix generalization such as the one in 63. Additionally, the distinction between the solutions of Quastel-Remenik and ours for KPZ fluctuations is highly reminiscent of the one for the $\mathrm{KdV} / \mathrm{KP}$ equations between solutions on the infinite line, where an extension to more singular initial conditions is required for KPZ [63], and quasi-periodic solutions involving compact Riemann surfaces, which appear to become non-compact for KPZ.

\subsection{Conclusions}

Several exact results for KPZ fluctuations with periodic boundaries have been reformulated in this paper in a compact way in terms of meromorphic differentials on Riemann surfaces related to polylogarithms with half-integer index. We believe that KPZ universality would benefit from a more systematic use of tools from algebraic geometry, especially more recent developments about non-compact Riemann surfaces of infinite genus 64]. Conversely, the very singular and universal nature of KPZ fluctuations suggests that objects appearing naturally for KPZ might also be of some interest in themselves for the field of algebraic geometry, especially when studying limits where the genus of Riemann surfaces goes to infinity.

A possible extension concerns the renormalization group flow $h_{\lambda}(x, t)$ from the equilibrium fixed point $\lambda \rightarrow 0$ to the KPZ fixed point $\lambda \rightarrow \infty$ considered in this paper. Whether the dynamics for finite $\lambda$ may also be expressed in a natural way in terms of Riemann surfaces is unclear at the moment. Hints of a duality [65] between the equilibrium fixed point in an infinite system and the KPZ fixed point for periodic boundaries, with halfinteger polylogarithms describing large deviations on both sides [66], suggest however the existence of a tight structure holding everything together. Partial exact results relevant to finite $\lambda$ with periodic boundaries have been obtained using the replica solution [37] of the KPZ equation and a weakly asymmetric exclusion process 67, 68] (see also 69, 70] for 
recent exact results with arbitrary asymmetry). The appearance of half-integer polylogarithms and $\zeta$ functions in related contexts of non-intersecting lattice paths [71], largest eigenvalues in the real Ginibre ensemble 72,73 and return probabilities for the symmetric exclusion process $[74$ and quantum spin chains $[75]$ on $\mathbb{Z}$ with domain wall initial condition might also have some connections to the equilibrium side of the duality.

Finally, the results of this paper are based on complicated asymptotics of Bethe ansatz formulas for TASEP in the limit where the number of lattice sites $L$ and the number of particles $N$ go to infinity with fixed density $N / L[39 \sqrt[42]{3}$. A natural question is whether TASEP with finite $L, N$ can already be described in terms of (finite genus) Riemann surfaces, so that the infinite genus Riemann surface $\mathcal{R}$ would emerge in a more transparent fashion in the large $L, N$ limit. Tools from algebraic geometry have already been used in the study of the more complicated Bethe equations for the asymmetric exclusion process with hopping in both directions $[76$ and the related XXZ spin chains with finite anisotropy [77,78]. The limit where the anisotropy of the spin chain goes to infinity, corresponding for the exclusion process to the TASEP limit, seems however a better starting point since the Bethe equations have a much simpler structure in that case, see e.g. 79,80 for related works.

\section{Riemann surfaces and ramified coverings}

In this section, we recall a few classical results about (compact) Riemann surfaces and ramified coverings. The various properties are illustrated using two examples: hyperelliptic Riemann surfaces $\mathcal{H}_{N}$, which are the proper domain of definition for square roots of polynomials, and Riemann surfaces $\mathcal{R}_{N}$ defined from sums of square roots, which have the topology of $N$-1-dimensional hypercubes. The Riemann surfaces $\mathcal{R}_{N}$ are finite genus analogues of the non-compact Riemann surfaces $\mathcal{R}$ introduced in section 3.8 and used for KPZ fluctuations in section 2. We refer to 8183 for good self-contained introductions to compact Riemann surfaces and ramified coverings.

\subsection{Analytic continuation and Riemann surfaces}

Let us consider a function $g_{0}$ analytic in the complex plane $\mathbb{C}$ except for the existence of branch cuts, i.e. paths in $\mathbb{C}$ across which $g_{0}$ is discontinuous. Extremities of branch cuts, called branch points, correspond to genuine singularities of the function $g_{0}$. The branch cuts themselves, on the other hand, are somewhat arbitrary. The domain of definition of $g_{0}$ can be extended by analytic continuation along paths crossing the branch cuts. In the favourable case considered in this paper, successive iterations of this procedure lead to functions $g_{i}, i \in I$ analytic in $\mathbb{C}$ except for the same branch cuts as $g_{0}$, such that the function $g_{i}$ on one side of a branch cut continues analytically to another function $g_{j}$ on the other side of the same branch cut. The collection of all branches $g_{i}$ represents a multivalued function. Multivalued basic special functions usually come with a standard choice for the principal value $g_{0}$.

Considering the domains of definition of the functions $g_{i}$ as distinct copies $\mathbb{C}_{i}$ of the complex plane 5 , the Riemann surface $\mathcal{M}$ for the function $g_{0}$ is built by gluing together the sheets $\mathbb{C}_{i}$ along branch cuts, and we use the notation $[z, i], z \in \mathbb{C}$ for the points on the

\footnotetext{
${ }^{5}$ For the sake of simplicity, we consider in this paper concrete Riemann surfaces, defined in terms of analytic continuation of functions and close to Riemann's original presentation, and not the more abstract modern formalism in terms of an atlas of charts and transition functions. All the Riemann surfaces considered in this paper can be understood as the natural domain of definition of some explicit multivalued function, and thus come with a natural ramified covering from the Riemann surface to $\mathbb{C}$.
} 


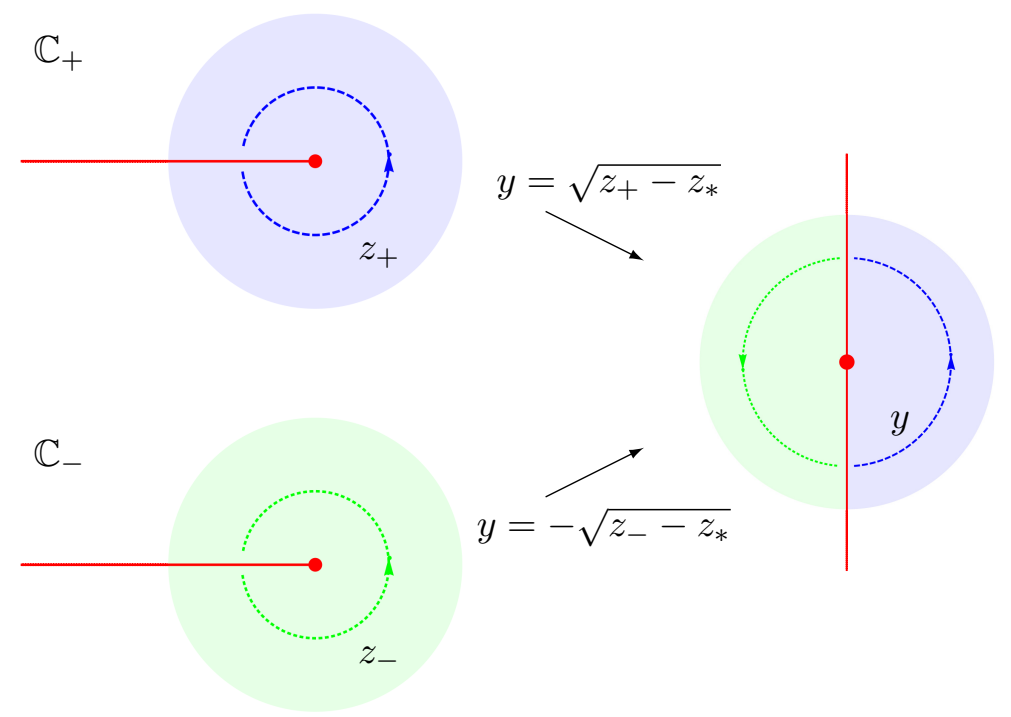

Figure 3: Neighbourhood of a point $q=\left[z_{*}, \mathbb{C}_{+}\right]=\left[z_{*}, \mathbb{C}_{-}\right]$in a Riemann surface (right) such that $z_{*}$ is a branch point of the function $g_{0}$ from which the Riemann surface is built. The neighbourhood is formed by gluing together along the branch cut originating from $q$ two half-disks obtained from taking the square root of full disks from the sheets $\mathbb{C}_{ \pm}$ (left). The complex numbers $z_{ \pm}$parametrize half a neighbourhood of $q$ in $\mathbb{C}_{ \pm}$. The local parameter $y$ at $q$ is a complex number that fully parametrizes the neighbourhood of $q$.

sheet $\mathbb{C}_{i}$ of $\mathcal{M}$. More precisely, let $z_{*}$ be a branch point of $g_{i}$ and $\gamma$ a branch cut issued from $z_{*}$. Calling by "left" and "right" the two sides of $\gamma$, we glue the left side of the cut in the sheet $\mathbb{C}_{i}$ to the right side of the cut in the sheet $\mathbb{C}_{j}$ if $g_{i}$ is analytically continued from the left to $g_{j}$ across the cut, and we glue the right side of the cut in the sheet $\mathbb{C}_{i}$ to the left side of the cut in the sheet $\mathbb{C}_{k}$ if $g_{i}$ is analytically continued from the right to $g_{k}$ across the cut. Additionally, the branch points $\left[z_{*}, i\right],\left[z_{*}, j\right],\left[z_{*}, k\right]$ of the sheets $\mathbb{C}_{i}, \mathbb{C}_{j}$, $\mathbb{C}_{k}$ represent a single point on $\mathcal{M},\left[z_{*}, i\right]=\left[z_{*}, j\right]=\left[z_{*}, k\right]$. The Riemann surface $\mathcal{M}$ is independent of the precise choice of branch cuts for $g_{0}$ : the branch cuts only determine a partition of $\mathcal{M}$ into sheets $\mathbb{C}_{i}$, and the notation $[z, i]$ for the points of $\mathcal{M}$ thus depends implicitly on the choice of branch cuts.

A function $g$ can then be defined on the Riemann surface $\mathcal{M}$ by $g([z, i])=g_{i}(z)$. Locally, the neighbourhood of any point of $\mathcal{M}$ looks like an open disk of $\mathbb{C}$, and the function $g$ is analytic there. This is obvious by construction, except around branch points where one needs to introduce a non-trivial local coordinate $y$ to parametrize the neighbourhood. We mainly consider in the following branch points $z_{*}$ of square root type, such that $g_{i}(z) \simeq \tilde{g}_{i}(z) \sqrt{z-z_{*}}$ when $z \rightarrow z_{*}$, where the $\tilde{g}_{i}$ are analytic and with a branch cut for the square root determined by the branch cuts of the $g_{i}$. A possible local parameter is then $y=\sqrt{z-z_{*}}$, and $g_{i}(z) \simeq y \tilde{g}_{i}\left(z_{*}+y^{2}\right)$ is indeed analytic around $y=0$. A neighbourhood of $z_{*}$ in $\mathcal{M}$ may be built using the local parameter $y$ by gluing together two half-disks as in figure 3 .

All the construction goes through in the presence of isolated poles, with analytic functions replaced by meromorphic functions. Additionally, it is often convenient to make Riemann surfaces compact by adding the points at infinity of the sheets, with appropriate local parameters ensuring that the neighbourhoods of these points are regular. In the simplest case where $g_{0}$ is a rational function without branch points and $\mathcal{M}$ is thus made of 

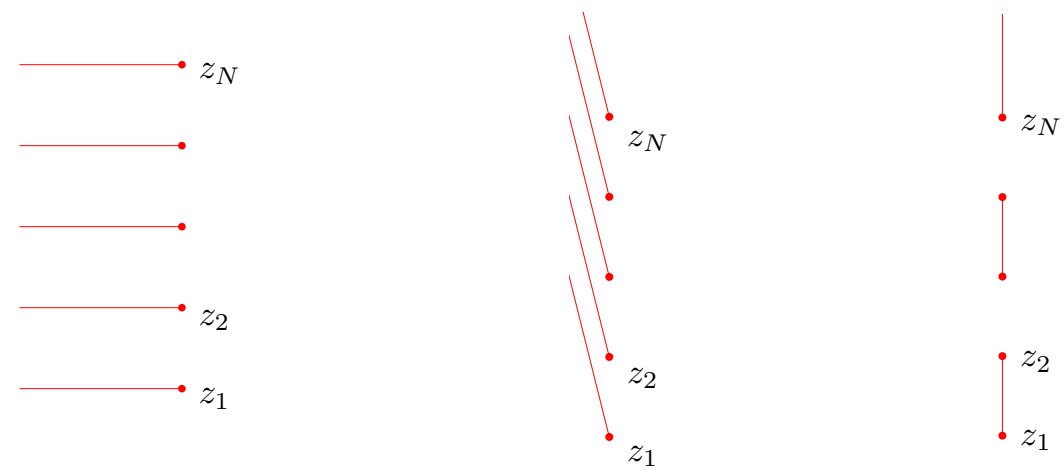

Figure 4: Three different choices of branch cuts (solid lines) for the function $h_{+}$defined in (27) with $N=5$. The branch points $z_{j}$ are represented by dots. The function $h_{+}$is multiplied by -1 after crossing any branch cut.

a single sheet $\mathbb{C}$, the associated compact Riemann surface is called 6 the Riemann sphere $\widehat{\mathbb{C}}=\mathbb{C} \cup\{\infty\}$.

\subsection{The Riemann surfaces $\mathcal{H}_{N}$ and $\mathcal{R}_{N}$}

We consider in this section two concrete examples of the construction above. Let $z_{1}, \ldots$, $z_{N}$ be distinct complex numbers, that are fixed in the following. We choose for simplicity of the pictures (and also because it will be the case of interest for KPZ) the $z_{j}$ 's to be purely imaginary and equally spaced, $\operatorname{Im} z_{1}<\ldots<\operatorname{Im} z_{N}$, but this choice is not essential here. We define the functions

$$
h_{+}(z)=\sqrt{z-z_{1}} \times \ldots \times \sqrt{z-z_{N}}
$$

and

$$
f_{\emptyset}(z)=\sqrt{z-z_{1}}+\ldots+\sqrt{z-z_{N}}
$$

of a complex variable $z$, which inherit branch cuts from the square roots (see respectively figures 4 and 5 for some possible choices of branch cuts). The functions $h_{+}$and $f_{\emptyset}$ can be extended by the procedure described in the previous section to analytic functions $h$ and $f$ defined on compact Riemann surfaces $\mathcal{H}_{N}$ and $\mathcal{R}_{N}$. The Riemann surface $\mathcal{H}_{N}$, called hyperelliptic and used here mainly for illustrative purpose, has links to the KdV equation discussed in section 2.6.3. The Riemann surface $\mathcal{R}_{N}$, on the other hand, is a simplified, finite genus version of the Riemann surface $\mathcal{R}$ introduced in section 3.8 , and in terms of which KPZ fluctuations are expressed in section 2.

We begin with the function $h_{+}$defined in $(27)$, analytic on a sheet called $\mathbb{C}_{+}$, with the choice of branch cuts on the left in figure 4. Analytic continuation across branch cuts gives $h_{-}=-h_{+}$, which lives on another sheet $\mathbb{C}_{-}$. The Riemann surface $\mathcal{H}_{N}$ is formed by the two sheets $\mathbb{C}_{ \pm}$glued together, and $h_{+}$extends analytically to a function $h$ defined on $\mathcal{H}_{N}$ by $h([z, \pm])=h_{ \pm}(z)$. Locally, the neighbourhood of any point of $\mathcal{H}_{N}$ looks like an open disk of $\mathbb{C}$ (see figure 3 for the neighbourhood of $\left[z_{j}, \pm\right]$ ), and the function $h$ is analytic there. The points at infinity $[\infty, \pm]$ are poles of the function $h$. A local parameter $y$ for these points is $y=z^{-1}$ if $N$ is even and $y=z^{-1 / 2}$ if $N$ is odd. In the former case, the poles of $h$ at $[\infty,+]$ and $[\infty,-]$, which are distinct points of $\mathcal{H}_{N}$, are of order $N / 2$. In the latter case, $\infty$ is a branch point of $h_{ \pm}$and the point $[\infty,+]=[\infty,-]$ is a pole of order $N$ of $h$. The function $h$ also has $N$ zeroes, the points $\left[z_{j},+\right]=\left[z_{j},-\right], j=1, \ldots, N$.

\footnotetext{
${ }^{6}$ Other notations for $\widehat{\mathbb{C}}$ include $\overline{\mathbb{C}}, \mathbb{C}_{\infty}$, or $\mathbb{P}^{1}(\mathbb{C}), \mathbb{C P}^{1}$ when interpreted as the complex projective line.
} 

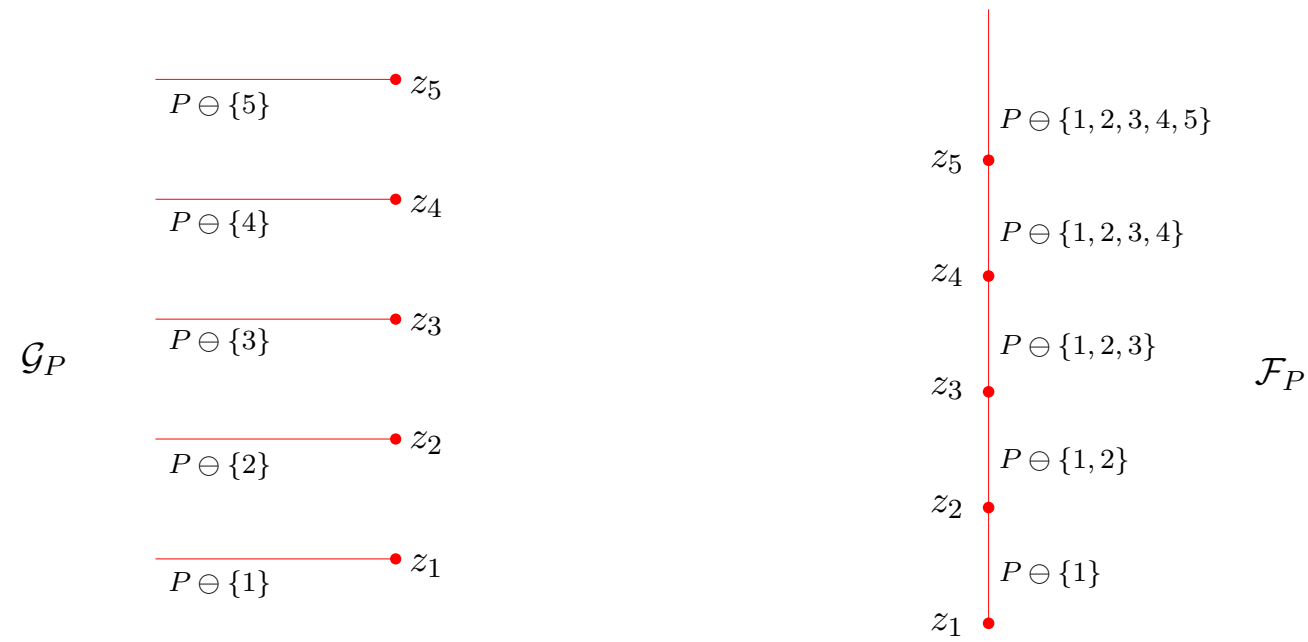

Figure 5: Two different choices of branch cuts (solid lines) for the function $f_{\emptyset}$ defined in (28) with $N=5$. The branch points are represented by dots. The sets labelling the sheet reached after crossing branch cuts from either side starting from the sheet labelled by $P \subset\{1,2,3,4,5\}$ is indicated near the branch cuts.

The total number of poles of $h$, counted with multiplicity, is thus equal to its number of zeroes. This is in fact a general property valid for any meromorphic function on a compact Riemann surface.

Compared to the hyperelliptic case discussed above, analytic continuations across branch cuts of $f_{\emptyset}$ defined in (28) have a richer structure, since all the square roots are independent: each one may change sign independently across branch cuts. The corresponding Riemann surface $\mathcal{R}_{N}$ is thus made of $2^{N}$ sheets labelled by sets of integers between 1 and $N, P \subset \llbracket 1, N \rrbracket$, indicating the square roots coming with a minus sign. It will be convenient in the following to distinguish two systems of sheets $\mathcal{G}_{P}$ and $\mathcal{F}_{P}$ partitioning $\mathcal{R}_{N}$, corresponding respectively to the choice of branch cuts on the left and on the right in figure 5. The points of $\mathcal{R}_{N}$ will be written as $\left[z, \mathcal{G}_{P}\right]$ or $\left[z, \mathcal{F}_{P}\right]$ when specifying the choice of sheets is needed, and simply as $[z, P]$ otherwise. The analytic function $f$ on $\mathcal{R}_{N}$ induced by $f_{\emptyset}$ is defined by $f([z, P])=f_{P}(z)$, with $f_{P}(z)=\sum_{j=1}^{N} \sigma_{j}(P) \sqrt{z-z_{j}}$ and

$$
\sigma_{a}(P)=\left\{\begin{array}{rl}
1 & a \notin P \\
-1 & a \in P
\end{array} .\right.
$$

The number of square roots that have changed sign in $f_{P}$ compared to $f_{\emptyset}$ is equal to the number $|P|$ of elements in $P$.

The connectivity of the sheets $\mathcal{G}_{P}$ and $\mathcal{F}_{P}$ in $\mathcal{R}_{N}$ following from analytic continuation can be expressed in terms of the symmetric difference operator $\ominus$, defined as union minus intersection:

$$
P \ominus Q=(P \cup Q) \backslash(P \cap Q) .
$$

The symmetric difference operator $\ominus$ is associative, commutative and verifies $P \ominus P=\emptyset$ and $7^{7} P \ominus Q+n=(P+n) \ominus(Q+n)$ for $n \in \mathbb{Z}$. A collection of sets closed under union, intersection and complement forms a group with the operation $\ominus$. The identity element is the empty set $\emptyset$, and the maps $\sigma_{a}$ act as group homomorphisms, $\sigma_{a}(P \ominus Q)=\sigma_{a}(P) \sigma_{a}(Q)$.

Crossing the branch cut associated to $z_{j}$ from the sheet $\mathcal{G}_{P}$ leads to $\mathcal{G}_{P \ominus\{j\}}$ (see figure 5), and one has the local parameters $y= \pm \sqrt{z-z_{j}}$ with the same half-disk construc-

\footnotetext{
${ }^{7}$ We choose symmetric difference to have precedence over addition, so that $P \ominus Q+n$ means $(P \ominus Q)+n$.
} 


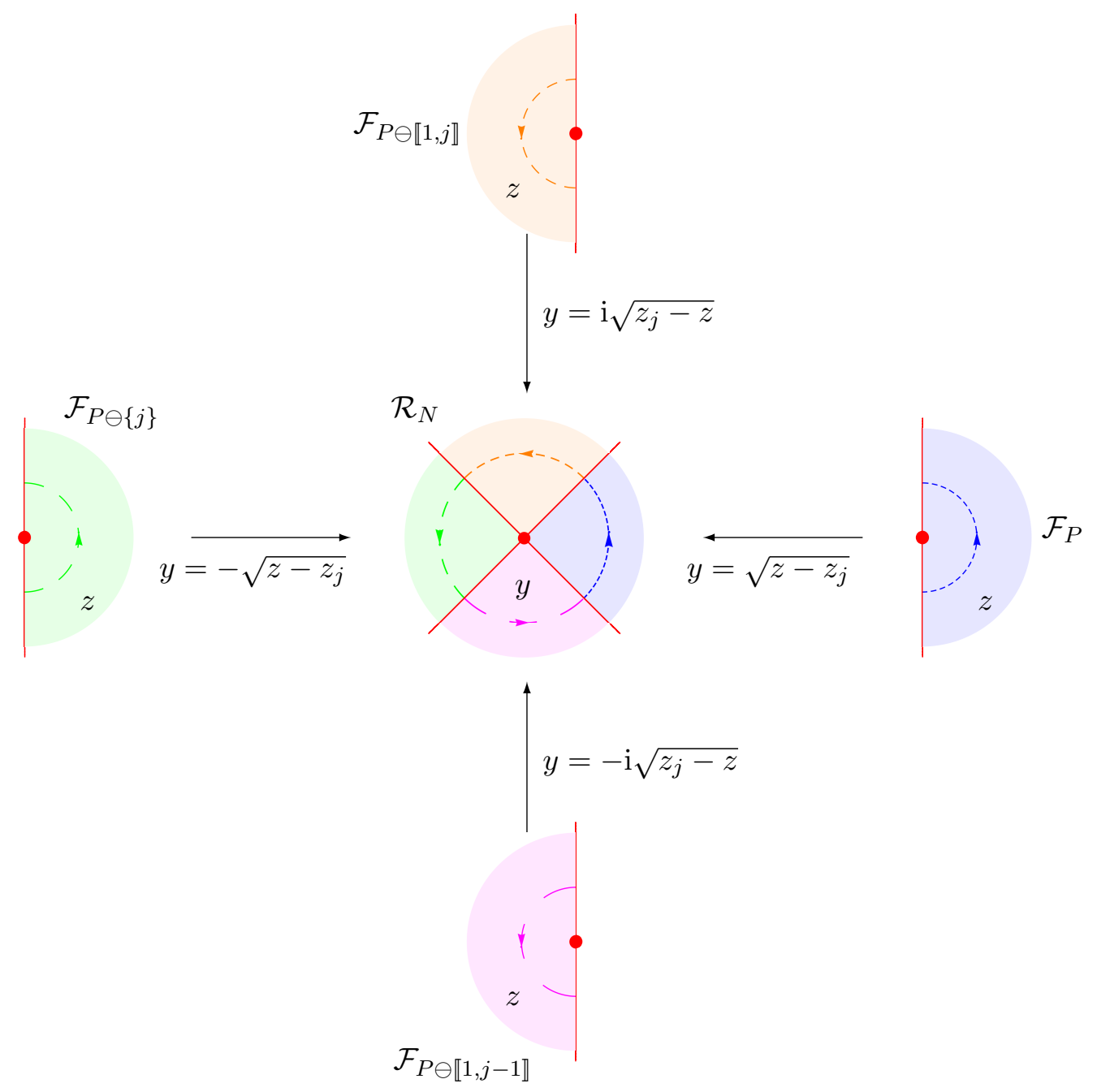

Figure 6: Neighbourhood of $q=\left[\left(z_{j}\right)_{\mathrm{r}}, \mathcal{F}_{P}\right]=\left[\left(z_{j}\right)_{1}, \mathcal{F}_{P \ominus \llbracket 1, j \rrbracket}\right]=\left[\left(z_{j}\right)_{\mathrm{r}}, \mathcal{F}_{P \ominus\{j\}}\right]=$ $\left[\left(z_{j}\right)_{1}, \mathcal{F}_{P \ominus \llbracket 1, j-1 \rrbracket}\right], 2 \leq j \leq N-1$ in $\mathcal{R}_{N}$, formed by gluing four quarter-disks obtained from taking the square root of half-disks in the sheets $\mathcal{F}_{P}, \mathcal{F}_{P \ominus \llbracket 1, j \rrbracket}, \mathcal{F}_{P \ominus\{j\}}$ and $\mathcal{F}_{P \ominus \llbracket 1, j-1 \rrbracket}$. The complex numbers $z$ parametrize quarters of neighbourhoods of $q$ in the various sheets. The local parameter $y$ fully parametrizes a neighbourhood of $q$, with $y=0$ corresponding to $q$. 

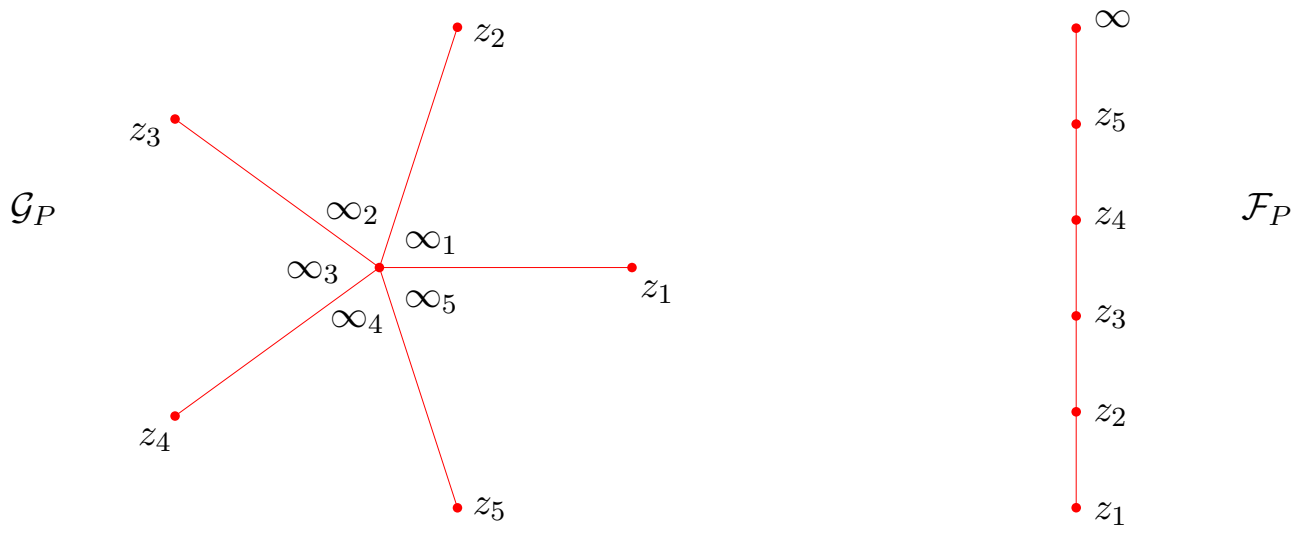

Figure 7: Compact representation of branch cuts for the sheets $\mathcal{G}_{P}$ (left) and $\mathcal{F}_{P}$ (right) of $\mathcal{R}_{5}$ after adding the points at infinity. The branch cuts are represented as straight lines for clarity, and distances are not meaningful.

tion of figure 3 as for $\mathcal{H}_{N}$ around the points $\left[z_{j}, \mathcal{G}_{P}\right]=\left[z_{j}, \mathcal{G}_{P \ominus\{j\}}\right]$. In the sheet $\mathcal{F}_{P}$ on the other hand, crossing the branch cut between $z_{j}$ and $z_{j+1}$ (with $z_{N+1}=\infty$ ) leads to $\mathcal{F}_{P \ominus \llbracket 1, j \rrbracket}$ (see figure 5). There, an additional difficulty for constructing local parameters is that the branch points $z_{j}, j=2, \ldots, N-1$ lie on branch cuts, and must thus be labelled by an additional index 1 or $\mathrm{r}$ depending on whether the point is on the left side (l) or the right side $(\mathrm{r})$ of the cut. A neighbourhood of $\left[\left(z_{j}\right)_{\mathrm{r}}, \mathcal{F}_{P}\right]=\left[\left(z_{j}\right)_{1}, \mathcal{F}_{P \ominus \llbracket 1, j \rrbracket}\right]=\left[\left(z_{j}\right)_{\mathrm{r}}, \mathcal{F}_{P \ominus\{j\}}\right]=$ $\left[\left(z_{j}\right)_{1}, \mathcal{F}_{P \ominus \llbracket 1, j-1 \rrbracket}\right]$ is constructed in figure 6 by gluing quarter disks together.

The points at infinity are branch points of the functions $f_{P}$. Considering the partition of $\mathcal{R}_{N}$ with sheets $\mathcal{F}_{P}$, one has $\left[\infty, \mathcal{F}_{P}\right]=\left[\infty, \mathcal{F}_{P \ominus \llbracket 1, N \rrbracket}\right]$, see figures 5 and 7 right. For the sheets $\mathcal{G}_{P}$, points at infinity can be reached from $N$ directions in figure 5 left, and one has to distinguish points $\left[\infty_{j}, \mathcal{G}_{P}\right], j=1, \ldots, N$, with the identifications $\left[\infty_{j}, \mathcal{G}_{P}\right]=$ $\left[\infty_{j-1}, \mathcal{G}_{P \ominus\{j\}}\right]$, see figure 7 left.

\subsection{Genus}

From a purely topological point of view, a Riemann surface is a two-dimensional connected manifold. In the case of a closed (i.e. without boundary), compact Riemann surface such as $\widehat{\mathbb{C}}, \mathcal{H}_{N}$ or $\mathcal{R}_{N}$ above, the manifold is fully characterized up to homeomorphisms (i.e. continuous deformations with continuous inverse) by a single non-negative integer, its genus $g$, corresponding to the maximal number of simple non-intersecting closed curves along which the manifold can be cut while still being connected. The case $g=0$ corresponds to a sphere, $g=1$ to a torus and $g \geq 2$ to a chain of $g$ tori glued together, see figure 8 ,

The additional complex structure of Riemann surfaces detailing how sheets are glued together gives more freedom, and two Riemann surfaces of the same genus are not necessarily isomorphic (i.e. there may not exist a holomorphic homeomorphism with holomorphic inverse transforming one into the other). The genus 0 case is an exception, for which a single Riemann surface exists up to isomorphism, the Riemann sphere $\widehat{\mathbb{C}}$. For genus 1, the equivalence classes up to isomorphism are indexed by a single complex number $\tau$ (defined up to modular transformations), such that the parallelogram with vertices $0,1,1+\tau, \tau$ becomes a torus when opposite sides are glued together. For higher genus $g \geq 2$, the moduli space of all Riemann surfaces is parametrized by $3 g-3$ complex parameters.

The Riemann surfaces $\mathcal{H}_{1}$ and $\mathcal{H}_{2}$ are both isomorphic to the Riemann sphere, while $\mathcal{H}_{3}$ and $\mathcal{H}_{4}$ are tori. More generally, the genus of the hyperelliptic Riemann surface $\mathcal{H}_{N}$ 

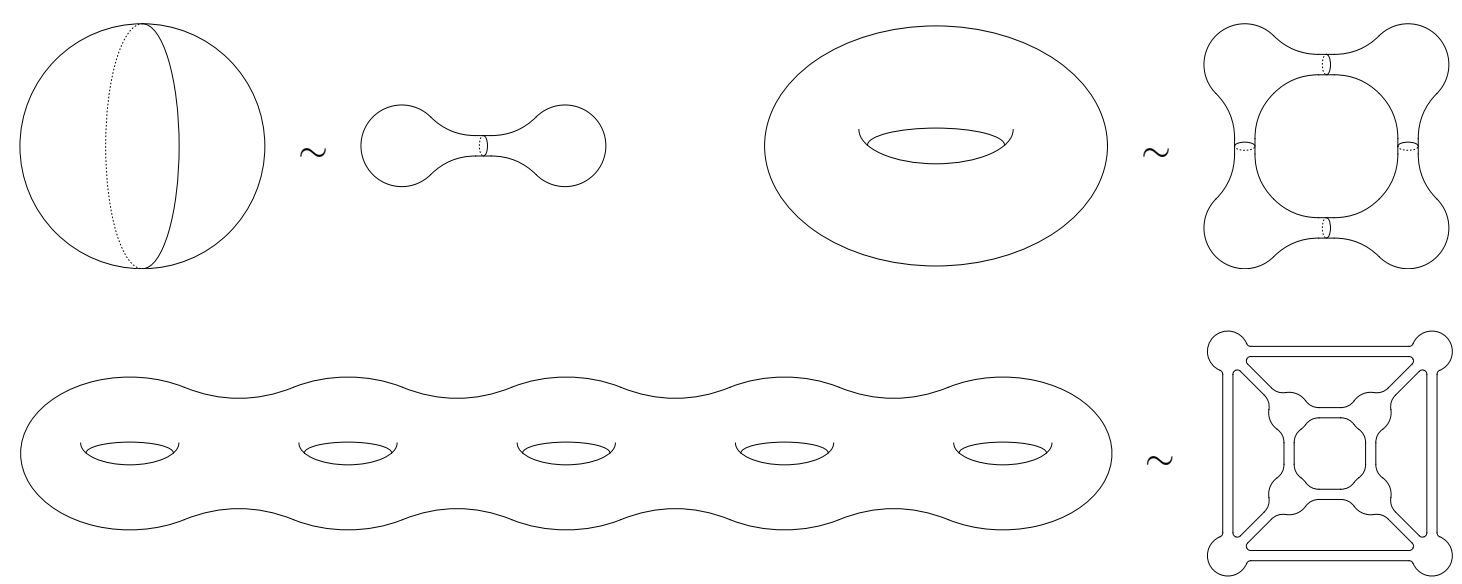

Figure 8: Sphere, torus, and surface with genus $g=5$, along with hypercubes of dimensions 1, 2, 3 made of spheres connected with cylinders that can be mapped to them by continuous deformations.

is known to be equal to either $(N-1) / 2$ or $(N-2) / 2$ depending on the parity of $N$. Similarly, $\mathcal{R}_{1}$ (which is exactly the same as $\mathcal{H}_{1}$ since $f_{\emptyset}=h_{+}$when $N=1$ ) and $\mathcal{R}_{2}$ (see figure 9) are also isomorphic to the Riemann sphere, while $\mathcal{R}_{3}$ is a torus, see figure 10. More generally, the genus $g_{N}$ of $\mathcal{R}_{N}$ grows exponentially fast with $N$, as

$$
g_{N}=1+(N-3) 2^{N-2},
$$

which can be proved by induction on $N$. Indeed, cutting $\mathcal{R}_{N}$ along a path joining $\left[z_{N}, \mathcal{F}_{P}\right]$ and $\left[\infty, \mathcal{F}_{P}\right]$ in every sheet $\mathcal{F}_{P}$ splits $\mathcal{R}_{N}$ into two disconnected pieces (corresponding to sheets $\mathcal{F}_{P}$ with either $N \in P$ or $\left.N \notin P\right)$, each component having $2^{N-1}$ boundaries corresponding to the cycles $\left[\infty, \mathcal{F}_{P}\right]=\left[\infty, \mathcal{F}_{P \ominus \llbracket 1, N \rrbracket}\right] \rightarrow\left[\left(z_{N}\right)_{1}, \mathcal{F}_{P \ominus \llbracket 1, N \rrbracket}\right]=\left[\left(z_{N}\right)_{\mathrm{r}}, \mathcal{F}_{P}\right] \rightarrow$ $\left[\infty, \mathcal{F}_{P}\right]$. Both pieces are homeomorphic to $\mathcal{R}_{N-1}$ with $2^{N-1}$ boundaries, which are connected two by two in $\mathcal{R}_{N}$. This leads to the recurrence relation $g_{N+1}=2 g_{N}+2^{N-1}-1$, since gluing together both pieces along a first boundary leads to a surface with twice the genus of $\mathcal{R}_{N-1}$, and each additional gluing adds a handle to the surface and hence increases the genus by 1 . We observe that the Riemann surface $\mathcal{R}_{N}$ is thus homeomorphic to a $N$-1-dimensional hypercube [84] whose nodes are spheres and edges cylinders connecting the spheres, see figure 8 .

\subsection{Ramified coverings}

Maps between Riemann surfaces acting as holomorphic functions on local parameters, called holomorphic maps, are a powerful tool in the study of Riemann surfaces. They allow in particular to define a notion of equivalence between Riemann surfaces having essentially the same complex structure: two Riemann surfaces are called isomorphic when there exists a bijective holomorphic map with holomorphic inverse between them.

Given two Riemann surfaces $\mathcal{M}$ and $\mathcal{N}$, a ramified covering (or branched covering, or simply covering map ${ }^{8}$ here for simplicity) such that $\mathcal{M}$ covers $\mathcal{N}$ (or $\mathcal{N}$ is covered by $\mathcal{M}$ ) is a non-constant holomorphic map from $\mathcal{M}$ to $\mathcal{N}$, which is then surjective by analyticity. Ramified coverings allow to relate complicated Riemann surfaces to simpler Riemann surfaces. In particular, for any Riemann surface $\mathcal{M}$, there exists at least one ramified covering from $\mathcal{M}$ to the Riemann sphere $\widehat{\mathbb{C}}$, i.e. a non-constant meromorphic function on $\mathcal{M}$. Conversely, defining a concrete Riemann surface $\mathcal{M}=\{[z, i], z \in \widehat{\mathbb{C}}, i \in I\}$ by gluing

\footnotetext{
${ }^{8}$ Not to be confused with the topological notion of a covering, which does not have ramification points.
} 

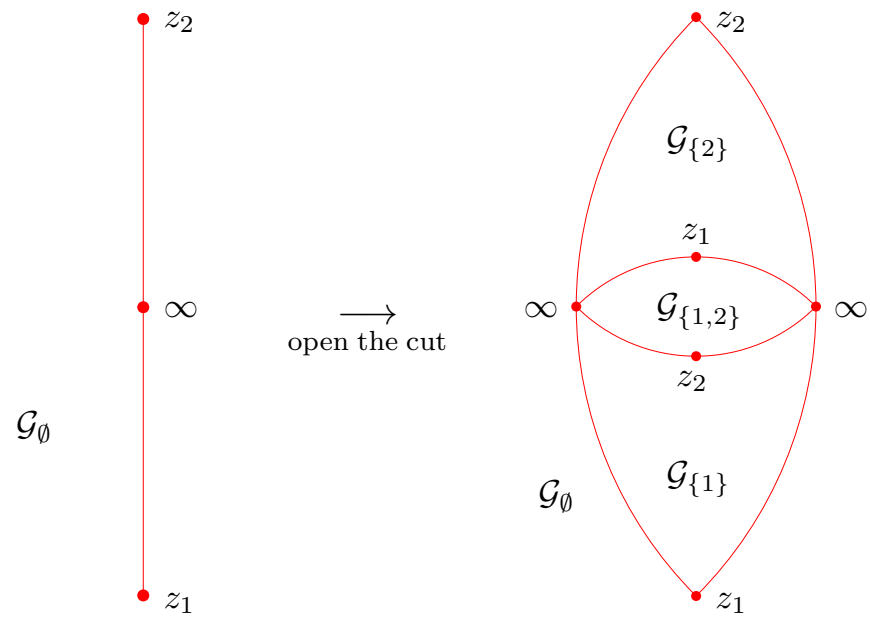

Figure 9: Representation of the surface corresponding to the Riemann surface $\mathcal{R}_{2}$. The sheet $\mathcal{G}_{\emptyset}$ is represented on the left, with a cut linking the points $\left[z_{1}, \mathcal{G}_{\emptyset}\right],\left[z_{2}, \mathcal{G}_{\emptyset}\right]$ and $\left[\infty, \mathcal{G}_{\emptyset}\right]$. Opening the cut, all the other sheets $\mathcal{G}_{\{1\}}, \mathcal{G}_{\{2\}}, \mathcal{G}_{\{1,2\}}$ fit within the opening. The graph made by the cuts of all sheets is planar, and $\mathcal{R}_{2}$ is isomorphic to the Riemann sphere $\widehat{\mathbb{C}}$.

$\begin{array}{llr} & \{1\} & \{1,2,3\} \\ \{1,2\} & \{1,3\}\end{array}$

Figure 10: Representation of the torus homeomorphic to the Riemann surface $\mathcal{R}_{3}$. Opposite sides of the fundamental domain represented by the region in the middle are identified. The connectivity of the sheets $\mathcal{G}_{P}$ partitioning $\mathcal{R}_{3}$ is represented by the labels $P \subset\{1,2,3\}$ of the sheets. 
domains of definition of branches $i \in I$ of a multivalued function gives the natural covering $\operatorname{map}[z, i] \mapsto z$.

Let $\rho: \mathcal{M} \rightarrow \mathcal{N}$ be a branched covering and $p \in \mathcal{M}$. Then, there exists a unique positive integer $e_{p} \in \mathbb{N}^{*}$ such that one can choose local coordinates $y$ and $z$ for the neighbourhoods around $p$ and $\rho(p)$ in such a way that $z=y^{e_{p}}$. The integer $e_{p}$ is called the ramification index of the point $p$ for the covering map $\rho$. A ramification point of $\rho$ is then a point $p \in \mathcal{M}$ such that $e_{p} \geq 2$, with $e_{p}=2$ corresponding to ramification points of square root type. Around such points, the mapping $\rho$ is not injective, and the inverse function $\rho^{-1}$ is multivalued. The branch points $q \in \mathcal{N}$ of $\rho$ are the images by $\rho$ of the ramification points $p \in \mathcal{M}$.

Ramification points form a discrete subset of $\mathcal{M}$. Furthermore, if $\mathcal{M}$ is compact, the number of branch points is finite, and so is the number of preimages of any $q \in \mathcal{N}$ by $\rho$. In that case, there exists a unique positive integer $d \in \mathbb{N}^{*}$, the degree of the branched covering, such that for any $q \in \mathcal{N}, d=\sum_{p \in \rho^{-1}(q)} e_{p}$. For generic points $q \in \mathcal{N}$ not branch points of $\rho$, the set of preimages $\rho^{-1}(q) \subset \mathcal{M}$ has exactly $d$ distinct elements.

The construction of a concrete Riemann surface $\mathcal{M}$ by gluing together a discrete number of copies $\widehat{\mathbb{C}}_{i}, i \in I$ of the Riemann sphere along branch cuts of some function, which we used above to define $\mathcal{H}_{N}$ and $\mathcal{R}_{N}$, naturally gives the ramified covering $[z, i] \mapsto z$ from $\mathcal{M}$ to $\widehat{\mathbb{C}}$.

New meromorphic function can be built from known ones using ramified coverings. Let $\rho: \mathcal{M} \rightarrow \mathcal{N}$ be a branched covering and $\varphi_{\mathcal{M}}, \varphi_{\mathcal{N}}$ meromorphic functions defined respectively on $\mathcal{M}$ and $\mathcal{N}$. Then, the composition $\varphi_{\mathcal{N}} \circ \rho$ is a meromorphic function on $\mathcal{M}$. For instance, the ramified covering $\rho:[z, P] \mapsto\left[z,(-1)^{|P|}\right]$ from $\mathcal{R}_{N}$ to $\mathcal{H}_{N}$ generates from the function $h$ on $\mathcal{H}_{N}$ defined from (27) the function $h \circ \rho$ meromorphic on $\mathcal{R}_{N}$, which is essentially the same function as $h$ but defined on a bigger space: $\mathcal{H}_{N}$ is only the "minimal" closed, compact Riemann surface on which $h_{ \pm}$can be extended to a meromorphic function.

Conversely, tracing over preimages of $\rho$ defines a function $\operatorname{tr}_{\rho} \varphi_{\mathcal{M}}$ as

$$
\left(\operatorname{tr}_{\rho} \varphi_{\mathcal{M}}\right)(q)=\sum_{\substack{p \in \mathcal{M} \\ \rho(p)=q}} \varphi_{\mathcal{M}}(p)
$$

which can be shown [85] to be meromorphic on $\mathcal{N}$. This can be illustrated by considering the covering map $\rho:[z, P] \mapsto z$ from $\mathcal{R}_{N}$ to $\widehat{\mathbb{C}}$. Starting with the function $f$ meromorphic on $\mathcal{R}_{N}$ defined from (28), all the square roots cancel in the $\operatorname{trace}$ and one has $\operatorname{tr}_{\rho} f=0$ which is indeed defined on $\widehat{\mathbb{C}}$. Less trivially, allowing essential singularities at infinity, the function $\left(\operatorname{tr}_{\rho} \mathrm{e}^{\lambda f}\right)(z)=2^{N} \prod_{j=1}^{N} \cosh \left(\lambda \sqrt{z-z_{j}}\right)$ is also analytic in $\mathbb{C}$.

The Riemann-Hurwitz formula gives a relation between ramification indices $e_{p}$ for a branched covering $\rho: \mathcal{M} \rightarrow \mathcal{N}$ of degree $d$ and the respective genus $g_{\mathcal{M}}, g_{\mathcal{N}}$ of the Riemann surfaces $\mathcal{M}$ and $\mathcal{N}$ :

$$
g_{\mathcal{M}}=d\left(g_{\mathcal{N}}-1\right)+1+\frac{1}{2} \sum_{p \in \mathcal{M}}\left(e_{p}-1\right),
$$

where only ramification points contribute to the sum. In particular, one has always $g_{\mathcal{M}} \geq g_{\mathcal{N}}$. Considering a triangulation of $\mathcal{M}$ with vertices at ramification points of $\rho$, the Riemann-Hurwitz formula is a simple consequence of the expression for the Euler characteristics $\chi=2-2 g$ in terms of the number of vertices, edges and faces of the triangulation.

The Riemann-Hurwitz formula allows one to recover the expression $(31)$ for the genus of $\mathcal{R}_{N}$. We introduce the ramified covering $[z, P] \mapsto z$ from $\mathcal{R}_{N}$ to $\widetilde{\mathbb{C}}$ for some choice of branch cuts. This covering has degree $d=2^{N}$, and its ramification points, all with 
ramification index 2 , are the $\left[z_{j}, P\right]$ and $[\infty, P]$. Each one is shared between two sheets (or four half-sheets, compare figures 3 and 6 ), so that the total number of ramification points is equal to $(N+1) 2^{N-1}$. Taking $g_{\mathcal{N}}=0$ in 33 since the target space is $\widehat{\mathbb{C}}$ gives again (31).

\subsection{Quotient under group action}

Quotients of Riemann surfaces by the action of their holomorphic automorphisms (i.e. bijective holomorphic maps from the Riemann surface to itself) generate new Riemann surfaces. Let $\mathcal{M}$ be a Riemann surface and $\mathfrak{h}$ a group of holomorphic automorphisms of $\mathcal{M}$ acting properly discontinuously on $\mathcal{M}$, i.e. for any point $p \in \mathcal{M}$, there exists a neighbourhood $U$ of $p$ in $\mathcal{M}$ such that the set $\{h \in \mathfrak{h}, h U \cap U \neq \emptyset\}$ is finite, with $h U=\{h q, q \in U\}$. The quotient $\mathcal{M} / \mathfrak{h}$ is then also a Riemann surface, whose points $q \in \mathcal{M} / \mathfrak{h}$ are identified to orbit of $p \in \mathcal{M}$ under the action of $\mathfrak{h}$, and the covering map $p \mapsto q$ from $\mathcal{M}$ to $\mathcal{M} / \mathfrak{h}$ is ramified at the fixed points of $\mathfrak{h}$.

Instead of considering the points of $\mathcal{M} / \mathfrak{h}$ as equivalence classes under the action of $\mathfrak{h}$, it is often convenient to choose a fundamental domain $\mathcal{F}$ in $\mathcal{M}$ such that $\{h \mathcal{F}, h \in \mathfrak{h}\}$ is a partition of $\mathcal{M}$. Then, $\mathcal{M} / \mathfrak{h}$ can be identified as $\mathcal{F}$ with additional boundary conditions. A genus 1 Riemann surface, which has the topology of a torus, can for instance be defined as the quotient of $\mathbb{C}$ by a group of translations in two directions, and the fundamental domain may always be chosen as a parallelogram whose opposite sides are glued together, see figure 10 .

Given two Riemann surfaces $\mathcal{M}, \mathcal{N}$ and a covering map $\rho$ from $\mathcal{M}$ to $\mathcal{N}$, a holomorphic automorphism $h$ of $\mathcal{M}$ is called a deck transformation for $\rho$ if $h$ is compatible with $\rho$, i.e. $\rho \circ h=\rho$. A deck transformation is fully determined by the permutation it induces on $\rho^{-1}(q)$ with $q \in \mathcal{N}$ not a branch point of $\rho$.

\subsection{Homotopy and homology}

Let $\mathcal{M}$ be a Riemann surface and $p \in \mathcal{M}$. Closed loops on $\mathcal{M}$ with base point $p$ are continuous paths on $\mathcal{M}$ starting and ending at $p$. The set of equivalence classes of such loops under homotopy (i.e. continuous deformations) forms a group $\pi_{1}(\mathcal{M})$ for the concatenation of the paths, called the first homotopy group (or fundamental group), which is independent from the base point up to group isomorphism.

Let $\mathcal{M}$ and $\mathcal{N}$ be two Riemann surfaces, $\rho: \mathcal{M} \rightarrow \mathcal{N}$ a covering map, $p \in \mathcal{M}$ not a ramification point of $\rho$ and $\gamma$ a continuous path in $\mathcal{N}$ starting at $\rho(p)$ and avoiding the branch points of $\rho$. The lift $\gamma \cdot p$ of $\gamma$ to the point $p$ is the unique path in $\mathcal{M}$ starting at $p$ whose image by $\rho$ is $\gamma$. Considering a partition of $\mathcal{M}$ into sheets $\mathbb{C}_{i}$ such that any point $q \in \mathcal{N}$ has a single preimage under $\rho$ in each sheet $\mathbb{C}_{i}$, we also write $\gamma \cdot \mathbb{C}_{i}$ for the lift of the path $\gamma \subset \mathcal{N}$ to the preimage $p \in \mathbb{C}_{i}$ of the starting point of $\gamma$. Even if $\gamma$ is a closed path on $\mathcal{N}$, the lift $\gamma \cdot \mathbb{C}_{i}$ is not necessarily a loop on $\mathcal{M}$, since its endpoint may be in any sheet $\mathbb{C}_{j}$, but loops from any equivalence class in $\pi_{1}(\mathcal{M})$ may be obtained by the lifting procedure.

Considering the example of the covering map $\rho:\left[z, \mathcal{G}_{P}\right] \mapsto z$ from $\mathcal{R}_{N}$ to $\widehat{\mathbb{C}}$, we call $\theta_{j}$ the loop in $\widehat{\mathbb{C}}$ encircling only the branch point $z_{j}$, once in the counter-clockwise direction. Then, the lift $\theta_{j}^{2} \cdot \mathcal{G}_{P}$ is homotopic to an empty loop on $\mathcal{R}_{N}$, see dashed path in figure 3 . Any loop on $\mathcal{R}_{N}$ may be written up to homotopy as $\theta_{j_{k}} \ldots \theta_{j_{1}} \cdot \mathcal{G}_{P}$, where each $\theta_{j}$ appears an even number of times in the product so that the final sheet $\mathcal{G}_{Q}, Q=P \ominus\left\{j_{1}\right\} \ominus \ldots \ominus\left\{j_{k}\right\}$ is the same as the initial sheet $\mathcal{G}_{P}$.

Considering several loops based at a point $p$ of a Riemann surface $\mathcal{M}$, the homotopy class of their product $\gamma$ may depend on the order of the loops in $\gamma$. On the other hand, the 
a)

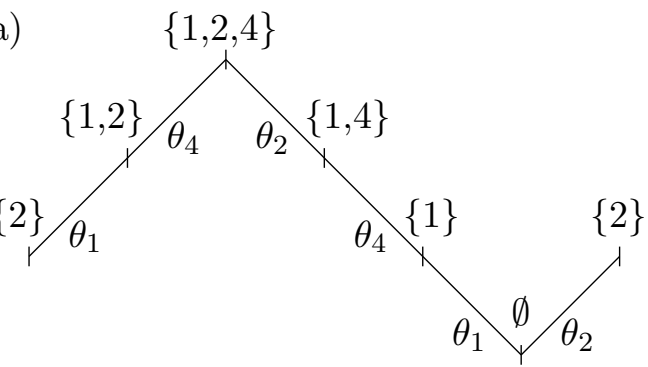

$$
\gamma=\theta_{2} \theta_{1} \theta_{4} \theta_{2} \theta_{4} \theta_{1} \cdot \mathcal{G}_{\{2\}}
$$

c)

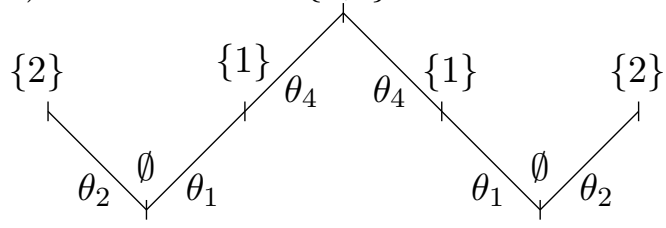

b)

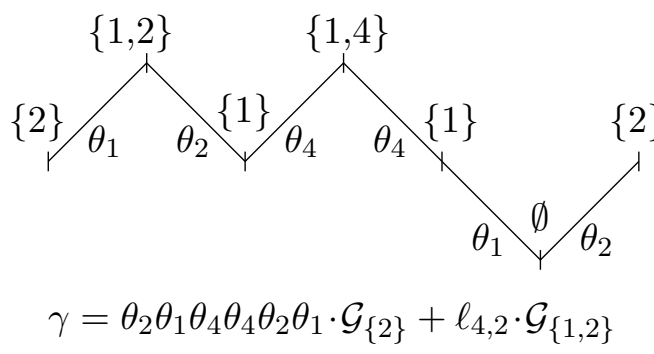

d)

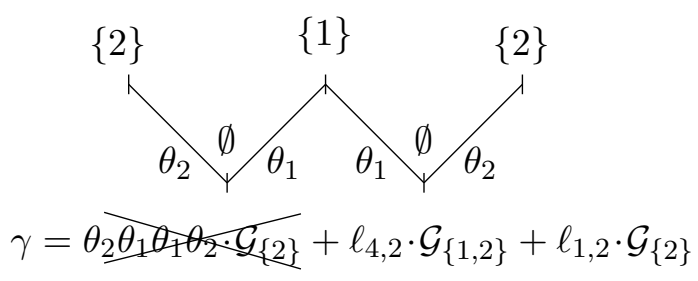

Figure 11: Homology class of a loop $\gamma=\theta_{j_{k}} \ldots \theta_{j_{1}} \mathcal{G}_{P}$ on the Riemann surface $\mathcal{R}_{N}$ rewritten as a combination of loops $\ell_{a, b} \cdot \mathcal{G}_{Q}$.

integral of a holomorphic differential (see next section) over $\gamma$ is independent of the order of the loops in $\gamma$. This motivates the definition of the first homology group $H_{1}(\mathcal{M}, \mathbb{Z})$, a commutative version of the fundamental group $\pi_{1}(\mathcal{M})$, for which an additive notation is used for the concatenation of loops. For a compact Riemann surface of genus $g$, it is known that a minimal set of generators of $H_{1}(\mathcal{M}, \mathbb{Z})$ must have $2 g$ elements.

An overcomplete set of generators for $H_{1}\left(\mathcal{R}_{N}, \mathbb{Z}\right)$ is given by the loops $\ell_{a, b} \cdot \mathcal{G}_{P}, 1 \leq$ $a<b \leq N, P \subset \llbracket 1, N \rrbracket$, with $\ell_{a, b}=\theta_{b} \theta_{a} \theta_{b} \theta_{a}$. Indeed, considering a general loop $\gamma=$ $\theta_{j_{k}} \ldots \theta_{j_{1}} \cdot \mathcal{G}_{P}$ of $\pi_{1}\left(\mathcal{R}_{N}\right)$, the sets $P=Q_{0}, Q_{1}, \ldots, Q_{k-1}, Q_{k}=P$ indexing the sheets $\mathcal{G}_{Q}$ crossed by $\gamma$ are such that two consecutive sets $Q_{i}, Q_{i+1}$ may only differ by a single element, and their cardinals $\left|Q_{i}\right|,\left|Q_{i+1}\right|$ differ by \pm 1 . Choosing an index $i$ such that $\left|Q_{i}\right|$ is a local maximum in the sequence, two situations can occur: if $Q_{i-1}=Q_{i+1}$, then $j_{i-1}=j_{i}$ and $\theta_{j_{i}} \theta_{j_{i-1}}$ is homotopic to the identity and can be erased. Otherwise $Q_{i-1} \neq Q_{i+1}$, and there exists $a, b \in \llbracket 1, N \rrbracket, a \neq b$ such that $Q_{i}$ contains both $a$ and $b, Q_{i-1}=Q_{i} \backslash\{b\}$ and $Q_{i+1}=Q_{i} \backslash\{a\}$. The loop then contains the factor $\theta_{b} \theta_{a}$, which can be replaced by $\theta_{a} \theta_{b}$, reducing the value of $\left|Q_{i}\right|$ by 2 at the price of adding $\ell_{a, b} \cdot Q_{i-1}$ to the loop, which has the form desired, see figure 11 for an example.

\subsection{Differential 1-forms}

Let $\mathcal{M}$ be a concrete Riemann surface equipped with a covering map $\rho:[z, i] \mapsto z$ from $\mathcal{M}$ to $\widehat{\mathbb{C}}$. A meromorphic differential $\omega$ on $\mathcal{M}$, also called an Abelian differential, is a differential 1-form such that at any $p=[z, i] \in \mathcal{M}$ away from branch points of $\rho$ one can write $\omega(p)=h_{i}(z) \mathrm{d} z$ with $h_{i}$ the branch of a meromorphic function $h$ on the sheet $\mathbb{C}_{i}$ of $\mathcal{M}$. At a ramification point $\left[z_{*}, i\right]$ of $\rho$ with ramification index $e \geq 2$, one has instead $\omega\left(\left[z_{*}, i\right]\right)=e y^{e-1} h_{i}\left(z_{*}+y^{e}\right) \mathrm{d} y$ in terms of the local coordinate $y, y^{e}=z-z_{*}$.

A meromorphic differential has a pole (respectively a zero) of order $n$ at $p=[z, i]$ away from ramification points if the function $h$ as above has a pole (resp. a zero) of order $n$ at $p$, and the residue of the pole is equal to the corresponding residue of $h_{i}$. For ramification 
points with local coordinate chosen as above, poles and zeroes of $\omega$ correspond to poles and zeroes of $e y^{e-1} h_{i}\left(z_{*}+y^{e}\right)$ at $y=0$, and the residue of a pole, equal to the corresponding residue at $y=0$, is independent from the choice of local coordinate. We observe that poles of $h$ at branch points may be cancelled in $\omega$ by the factor $y^{e-1}$ if the order of the pole is strictly lower than the ramification index. For a compact Riemann surface of genus $g$, the degree of a meromorphic differential, i.e. the total number of zeroes minus the total number of poles counted with multiplicity, is equal to $2 g-2$.

It is convenient to classify meromorphic differentials into three kinds depending on their poles. Meromorphic differentials of the first kind, also called holomorphic differentials, correspond to the special case where the differential has no poles. Holomorphic differentials are closed, i.e. the integral of a holomorphic differential on a path on $\mathcal{M}$ does not change if the path is deformed continuously while its endpoints are kept fixed. Equivalently, the integral of a holomorphic differential over a loop depends only on the equivalence class of the loop under homology. In particular, if the loop is homologous to $0 \in H_{1}(\mathcal{M}, \mathbb{Z})$, the integral is equal to $0 \in \mathbb{C}$ even though the loop may not be homotopic to an empty loop. Holomorphic differentials form a vector space $H^{1}(\mathcal{M}, \mathbb{C})$ of dimension $2 g$, dual to the first homology group $H_{1}(\mathcal{M}, \mathbb{Z})$, and are the basic ingredients to build theta functions, a fundamental object in the theory of compact Riemann surfaces in terms of which $\tau$ functions for the KdV equation can for instance be built. For the Riemann surface $\mathcal{R}_{N}$, a basis of holomorphic differentials is given by the $\omega_{Q, k}, Q \subset \llbracket 1, N \rrbracket, k$ integer with $0 \leq k \leq$ $(|Q|-3) / 2$, equal at $[z, P]$ not a ramification point to $\omega_{Q, k}([z, P])=z^{k} \mathrm{~d} z / \prod_{\ell \in Q} \sqrt{z-z_{\ell}}$. One can check that there are indeed $2 g_{N}$ holomorphic differentials $\omega_{Q, k}$, each one having degree $2 g_{N}-2$ with $g_{N}$ given by (31).

Meromorphic differentials of the second and third kind have poles. Meromorphic differentials of the second kind only have multiple poles with no residues, and are thus closed like holomorphic differentials. Meromorphic differentials of the third kind, on the other hand, also have poles with non-zero residue, and the integral over a small loop with winding number 1 around a pole is equal to $2 \mathrm{i} \pi$ times the residue of the pole, like for meromorphic functions on $\mathbb{C}$.

As with meromorphic functions, the trace of a meromorphic differential $\omega$ on $\mathcal{M}$ with respect to a covering map $\rho$ from $\mathcal{M}$ to $\mathcal{N}$ is defined as a sum over all preimages of $\rho$,

$$
\left(\operatorname{tr}_{\rho} \omega\right)(q)=\sum_{\substack{p \in \mathcal{M} \\ \rho(p)=q}} \omega(p) .
$$

If $\omega$ is holomorphic on $\mathcal{M}, \operatorname{tr}_{\rho} \omega$ is then holomorphic on $\mathcal{N}$ [85]. This observation is crucial for the application to KPZ in section 2 in order to move freely contours of integration between the left side and the right side of the cylinder $\mathcal{C}$.

\subsection{Infinite genus limit}

We consider in this section an infinite genus version $\mathcal{R}$ of the Riemann surface $\mathcal{R}_{N}$. Riemann surfaces $\check{\mathcal{R}}$ and $\mathcal{R}^{\Delta}$ in terms of which KPZ fluctuations are described in section 2 are constructed as quotients of $\mathcal{R}$ under the action of groups of holomorphic automorphisms.

\subsubsection{Riemann surface $\mathcal{R}$}

The Riemann surface $\mathcal{R}$ can be understood informally as a limit $N \rightarrow \infty$ of $\mathcal{R}_{N}$ with the choice of branch points $2 \mathrm{i} \pi(\mathbb{Z}+1 / 2)$ for the covering map $[v, P] \mapsto v$. Topologically, $\mathcal{R}$ is thus an infinite dimensional hypercube made of spheres connected by cylinders, see figure 8 


$$
\begin{aligned}
{[5 \mathrm{i} \pi, P] } & \cdot \\
{[3 \mathrm{i} \pi, P] } & \cdot \mathbb{C}_{P \ominus B_{2}} \\
\mathbb{C}_{P} & \mathbb{C}_{P \ominus B_{1}} \\
{[\mathrm{i} \pi, P] } & \cdot \\
{[-\mathrm{i} \pi, P] } & \cdot \mathbb{C}_{P \ominus B_{-1}} \\
{[-3 \mathrm{i} \pi, P] } & \cdot \mathbb{C}_{P \ominus B_{-2}} \\
& {[-5 \mathrm{i} \pi, P] }
\end{aligned}
$$

Figure 12: Choice of branch cuts (red, vertical lines) partitioning the Riemann surface $\mathcal{R}$ into the sheets $\mathbb{C}_{P}, P \sqsubset \mathbb{Z}+1 / 2$. The ramification points of the covering map $\Pi$ from $\mathcal{R}$ to the infinite cylinder $\mathcal{C}$ are represented with red dots. The connectivity of the sheets is indicated near the cuts, with $B_{n}$ defined in (35) and $\ominus$ the symmetric difference operator from $(30)$.

for finite genus analogues $\mathcal{R}_{N}$. The Riemann surface $\mathcal{R}$ is understood more concretely in section 4.4 as a natural domain of definition for polylogarithms with half-integer index, which generalize the finite sum of square roots $(28)$ on $\mathcal{R}_{N}$.

Branch cuts are chosen such that $\mathcal{R}$ is partitioned into infinitely many sheets $\mathbb{C}_{P}$ indexed by subsets $P$ of $\mathbb{Z}+1 / 2{ }^{9}$, copies of the complex plane slit along the cut $(-\mathrm{i} \infty,-\mathrm{i} \pi] \cup$ $[i \pi, i \infty)$ with the points on the cut belonging by convention to e.g. the left part of the cut. Introducing for $n \in \mathbb{Z}$ the sets

$$
\begin{array}{ll}
B_{0}=\emptyset & n=0 \\
B_{n}=\{1 / 2,3 / 2, \ldots, n-1 / 2\} & n>0 \\
B_{n}=\{n+1 / 2, \ldots,-3 / 2,-1 / 2\} & n<0
\end{array},
$$

the sheets $\mathbb{C}_{P}$ and $\mathbb{C}_{P \ominus B_{n}}$ are glued together along both sides of the cut $2 \mathrm{i} \pi(n-1 / 2, n+1 / 2)$ 10 with $\ominus$ the symmetric difference operator defined in $(30)$, see figure 12

Since the branch points $2 \mathrm{i} \pi a, a \in \mathbb{Z}+1 / 2$ are equally spaced, there exists a covering map $\Pi:[v, P] \mapsto v-2 \mathrm{i} \pi\left[\frac{\operatorname{Im} v}{2 \pi}\right]$, with $\left[\frac{\operatorname{Im} v}{2 \pi}\right]$ the integer closest to $\frac{\operatorname{Im} v}{2 \pi}$, from $\mathcal{R}$ to the infinite cylinder $\mathcal{C}=\{v \in \mathbb{C}, v \equiv v+2 \mathrm{i} \pi\}$, see figure 1. Using the covering map $\lambda: v \mapsto \mathrm{e}^{v}$, the infinite cylinder is completely equivalent to $\mathbb{C}^{*}=\mathbb{C} \backslash\{0\}$, i.e. the Riemann sphere punctured twice $\mathbb{C}^{*}=\widehat{\mathbb{C}} \backslash\{0, \infty\}$.

A subtle issue concerns whether sheets indexed by infinite sets $P$ should be considered when building $\mathcal{R}$, as such sheets can not be reached from sheets indexed by finite sets without crossing infinitely many branch cuts. Since only sheets indexed by finite sets appear in our formulas for KPZ fluctuations in section 2, we avoid this issue completely and define $\mathcal{R}$ by gluing together only the sheets indexed by finite sets $P \sqsubset \mathbb{Z}+1 / 2$ with cardinal $|P|$. A related issue concerns the status of the points at infinity in $\mathcal{R}$. Unlike in $\mathcal{R}_{N}$, we may not add these points to $\mathcal{R}$ since they correspond to an accumulation of

\footnotetext{
${ }^{9}$ The choice of sets of half-integers instead of integers to label the sheets is for symmetry between analytic continuations above and under the real axis for the functions of section 4

${ }^{10}$ The choice of "vertical" branch cuts like for the sheets $\mathcal{F}_{P}$ of $\mathcal{R}_{N}$ instead of "horizontal" branch cuts like for the sheets $\mathcal{G}_{P}$ is for better compatibility with translations by integer multiples of $2 \mathrm{i} \pi$ later on.
} 
ramification points for the covering map $[z, P] \mapsto z$. The two points at infinity on each sheet (on the left side and on the right side of the cut) are thus considered as punctures of $\mathcal{R}$, i.e. infinitesimal boundaries.

Homology classes of loops on $\mathcal{R}$ avoiding the punctures are generated by the same loops $\ell_{a, b} \cdot P, a<b \in \mathbb{Z}+1 / 2$ as the ones defined for the finite genus analogue $\mathcal{R}_{N}$ in section 3.6. with $\theta_{a}$ now encircling $2 \mathrm{i} \pi a$. Additionally, paths between punctures play an important role for explicit computation of analytic continuations between the various sheets $\mathbb{C}_{P}$ for the functions needed to express KPZ fluctuations.

\subsubsection{Riemann surface $\check{\mathcal{R}}$}

Anticipating the fact that some functions on $\mathcal{R}$ defined in section 4 have special symmetries when their variable $[v, P] \in \mathcal{R}$ is replaced by $[v+2 \mathrm{i} \pi, P]$, we are lead to define also the quotient $\check{\mathcal{R}}$ of $\mathcal{R}$ under the action of a group of translations.

Let us consider the bijective operators $T_{1 \mid \mathrm{r}}{ }^{11}$ acting on finite sets $P \sqsubset \mathbb{Z}+1 / 2$ by $T_{1} P=P+1$ and $T_{\mathrm{r}} P=(P+1) \ominus\{1 / 2\}$. For any $m \in \mathbb{Z}$, the iterated composition of these operators is given by

$$
\begin{aligned}
& T_{1}^{m} P=P+m \\
& T_{\mathrm{r}}^{m} P=(P+m) \ominus B_{m} .
\end{aligned}
$$

The operators $T_{1 \mid \mathrm{r}}$ generate two groups $G_{1 \mid \mathrm{r}}=\left\{T_{1 \mid \mathrm{r}}^{m}, m \in \mathbb{Z}\right\}$ acting on $\mathbb{Z}+1 / 2$. The empty set is invariant under $T_{1}$, and $\{\emptyset\}$ thus constitutes an orbit under the action of $G_{1}$. The other orbits under the action of $G_{1}$ are the infinite collections of sets $P$ obtained from one another by shifting all the elements by an integer $m$, and equivalence classes of sets in the same orbit may be labelled by e.g. sets $P$ whose smallest element is equal to $1 / 2$. Each orbit under the action of $G_{\mathrm{r}}$, on the other hand, contains infinitely many elements. The identity

$$
\left|(P+m) \ominus B_{m}\right|_{+}-\left|(P+m) \ominus B_{m}\right|_{-}=|P|_{+}-|P|_{-}+m,
$$

with $|P|_{+}$(respectively $|P|_{-}$) denoting the number of positive (resp. negative) elements of the set $P$, indicates that each orbit under the action of $G_{\mathrm{r}}$ contains a single element $P$ with $|P|_{+}=|P|_{-}$, which may be used to label the equivalence class.

Let us now consider the map $\mathcal{T}$ defined on the left side of the sheet $\mathbb{C}_{\emptyset}$ of $\mathcal{R}$ by $\mathcal{T}[v, \emptyset]=[v+2 \mathrm{i} \pi, \emptyset], \operatorname{Re} v<0$. The map $\mathcal{T}$ can be extended from the left side of $\mathbb{C}_{\emptyset}$ to $\mathcal{R}$ by lifting as follows: let $v_{0} \in \mathbb{C}$ with $\operatorname{Re} v_{0}<0$ and $\gamma=\{\gamma(t), 0 \leq t \leq 1\}$, a path contained in $\mathbb{C} \backslash 2 \mathrm{i} \pi(\mathbb{Z}+1 / 2)$ starting at $\gamma(0)=v_{0}$. The lifts $\gamma \cdot \emptyset$ and $(\gamma+2 \mathrm{i} \pi) \cdot \emptyset$ are paths on $\mathcal{R}$ starting respectively at $\left[v_{0}, \emptyset\right]$ and $\left[v_{0}+2 \mathrm{i} \pi, \emptyset\right]$. Calling $[v, P]$ the endpoint of $\gamma \cdot \emptyset$, induction on the number of times $\gamma$ crosses the imaginary axis implies that the endpoint of $(\gamma+2 \mathrm{i} \pi) \cdot \emptyset$ is $[v+2 \mathrm{i} \pi, P+1]$ if $\operatorname{Re} v<0$ and $[v+2 \mathrm{i} \pi,(P+1) \ominus\{1 / 2\}]$ if $\operatorname{Re} v>0$, independently of $v_{0}$ and $\gamma$. Checking carefully what happens when $v$ is on the imaginary axis, especially in a neighbourhood of points of the form $\left[(2 \mathrm{i} \pi a)_{1 \mid \mathrm{r}}, P\right]$, we observe that the map $\mathcal{T}$ defined by $\mathcal{T}([v, P])=[v+2 \mathrm{i} \pi, P+1]$ when $\operatorname{Re} v<0, \mathcal{T}([v, P])=[v+2 \mathrm{i} \pi,(P+1) \ominus\{1 / 2\}]$ when $\operatorname{Re} v>0$ and extended by continuity to $\operatorname{Re} v=0$ is a homeomorphism of $\mathcal{R}$, and hence an automorphism since it is locally holomorphic. The map $\mathcal{T}$ is additionally a deck transformation for the covering map $\Pi$ from $\mathcal{R}$ to the infinite cylinder $\mathcal{C}$. The iterated composition $\mathcal{T}^{m}, m \in \mathbb{Z}$ is given by

$$
\mathcal{T}^{m}([v, P])= \begin{cases}{\left[v+2 \mathrm{i} \pi m, T_{1}^{m} P\right]} & \operatorname{Re} v<0 \\ {\left[v+2 \mathrm{i} \pi m, T_{\mathrm{r}}^{m} P\right]} & \operatorname{Re} v>0\end{cases}
$$

\footnotetext{
${ }^{11}$ We write $1 \mid \mathrm{r}$ in the following as a shorthand for either the left side $\mathrm{l}$ or the right side $\mathrm{r}$ of a cut.
} 

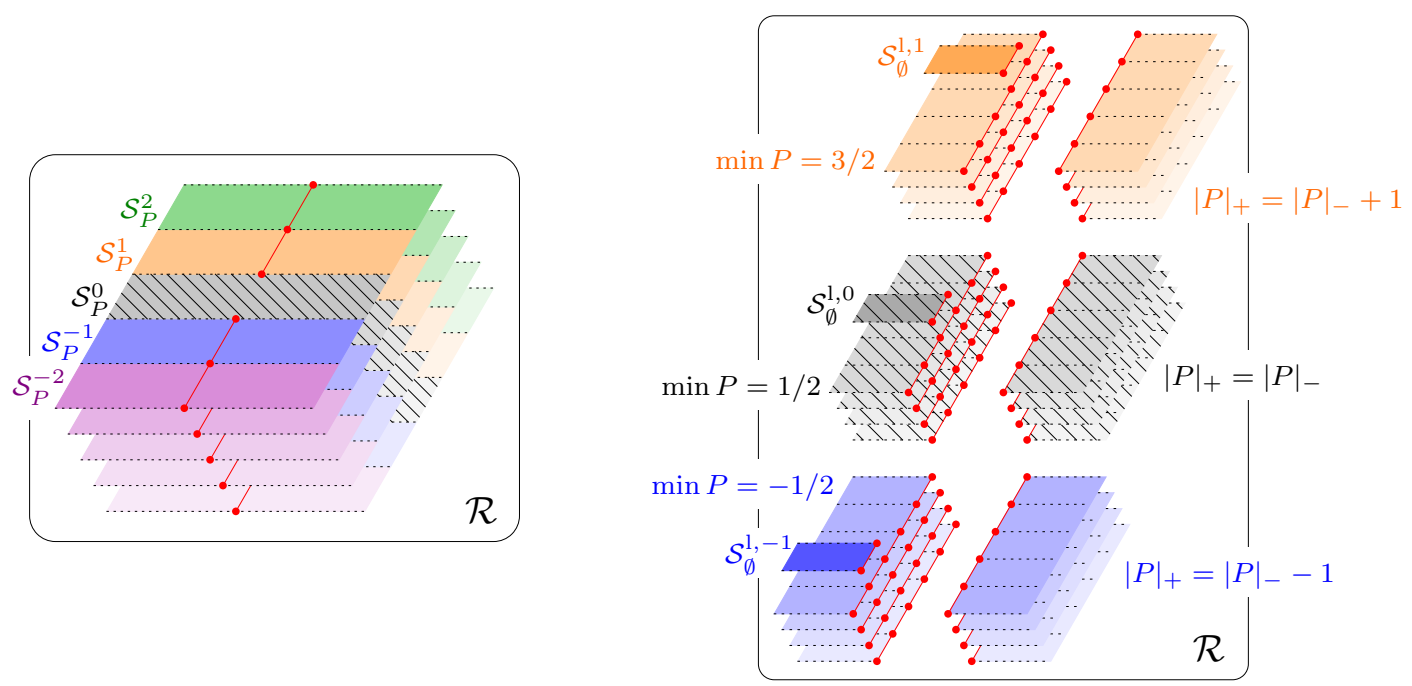

Figure 13: Two choices for a fundamental domain of $\mathcal{R}$ under the action of $\mathfrak{\mathfrak { g }}$, from which $\check{\mathcal{R}}=\mathcal{R} / \check{\mathfrak{g}}$ is built. The fundamental domain is the hatched portion. How sheets are glued together along branch cuts (red lines, with dots for the branch points) is not represented for clarity. The picture on the left represents all sheets $\mathbb{C}_{P}, P \sqsubset \mathbb{Z}+1 / 2$ above one another, with the fundamental domain made of the infinite strips $\mathcal{S}_{P}^{0}$ corresponding to points $[v, P]$ with $-\pi<\operatorname{Im} v \leq \pi$. The picture on the right represents half-sheets $\mathbb{C}_{P}^{l}$ grouped according to the value of $\min P$ (if $P \neq \emptyset$; the half-sheet $\mathbb{C}_{\emptyset}^{l}$ is cut into half-infinite strips $\mathcal{S}_{\emptyset}^{1, m}$ ) and half-sheets $\mathbb{C}_{P}^{\mathrm{r}}$ grouped by the value of $|P|_{+}-|P|_{-}$.

with $T_{1 \mid \mathrm{r}}^{m}$ defined in $(36)$. The group $\check{\mathfrak{g}}=\left\{\mathcal{T}^{m}, m \in \mathbb{Z}\right\}$ acts properly discontinuously on $\mathcal{R}$, and we call $\check{\mathcal{R}}=\mathcal{R} / \check{\mathfrak{g}}$ the Riemann surface quotient of $\mathcal{R}$ under the action of $\check{\mathfrak{g}}$.

The Riemann surface $\check{\mathcal{R}}$ may be partitioned into half-sheets $\mathbb{C}_{P}^{\mathrm{l|r}}$ by taking as a fundamental domain for $\mathfrak{g}$ the left side of the sheets $\mathbb{C}_{P}$ of $\mathcal{R}$ with some choice of representatives $P$ for the orbits under the action of $G_{1}$ plus the right side of the sheets $\mathbb{C}_{P}$ with some choice $P$ of representatives for the orbits under the action of $G_{\mathrm{r}}$, with the additional identification $v=v+2 \mathrm{i} \pi$ for the sheet $\mathbb{C}_{\emptyset}^{l}$ since $\{\emptyset\}$ is an orbit under the action of $G_{1}$, see figure 13 right. How sheets are glued together along the cuts depends on which representatives are chosen for the orbits.

$\begin{array}{ccc}\mathcal{S}_{P-1}^{0} & \cdot & \mathcal{S}_{P \ominus\{1 / 2\}-1}^{0} \\ & \mathcal{S}_{P}^{0} & \\ & \bullet & \mathcal{S}_{(P+1) \ominus\{1 / 2\}}^{0}\end{array}$

Figure 14: Connectivity of the infinite strips $\mathcal{S}_{P}^{0}, P \sqsubset \mathbb{Z}+1 / 2$ partitioning the Riemann surface $\check{\mathcal{R}}$. The red dots represent ramification points for the covering map $\check{\rho}$ sending all the strips to the infinite cylinder $\mathcal{C}$. 


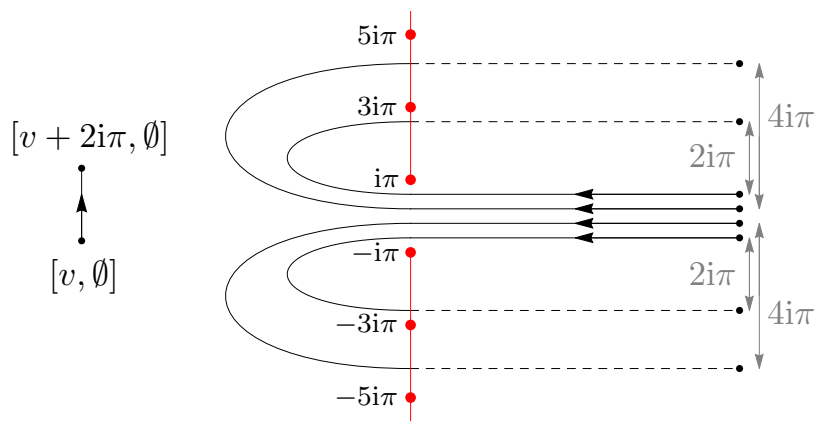

Figure 15: Examples of paths on $\mathcal{R}$ which are also closed loops on $\check{\mathcal{R}}$. The solid curves belong to $\mathbb{C}_{\emptyset}$ and the dashed lines to $\mathbb{C}_{B_{m}}, m=2,1,-1,-2$ from top to bottom.

Alternatively, we consider a partition of $\mathcal{R}$ into half-infinite strips

$$
\begin{aligned}
& \mathcal{S}_{P}^{\mathrm{l}, m}=\{[v, P], \operatorname{Re} v \leq 0,2 \pi(m-1 / 2)<\operatorname{Im} v \leq 2 \pi(m+1 / 2)\} \\
& \mathcal{S}_{P}^{\mathrm{r}, m}=\{[v, P], \operatorname{Re} v>0,2 \pi(m-1 / 2)<\operatorname{Im} v \leq 2 \pi(m+1 / 2)\} .
\end{aligned}
$$

The domain $\mathcal{S}_{P}^{m}=\mathcal{S}_{P}^{\mathrm{l}, m} \cup \mathcal{S}_{P}^{\mathrm{r}, m}$, contained in the sheet $\mathbb{C}_{P}$, is connected only when $m=0$, see figure 13 left. A fundamental domain for the action of $\mathfrak{\mathfrak { g }}$ in $\mathcal{R}$ may be chosen as the $\mathcal{S}_{P}^{0}$ indexed by all $P \sqsubset \mathbb{Z}+1 / 2$. In the Riemann surface $\check{\mathcal{R}}$, the upper part $\operatorname{Im} v=\pi$ of $\mathcal{S}_{P}^{0}$ is glued to the lower part $\operatorname{Im} v \rightarrow-\pi$ of $\mathcal{S}_{P-1}^{0}$ on the left side, while the upper part of $\mathcal{S}_{P}^{0}$ is glued to the lower part of $\mathcal{S}_{P \ominus\{1 / 2\}-1}^{0}$ on the right side, see figure 14 . This choice of a fundamental domain naturally defines the covering maps $\check{\Pi}:[v, P] \mapsto\left[v-2 \mathrm{i} \pi\left[\frac{\operatorname{Im} v}{2 \pi}\right], P\right]$ from $\mathcal{R}$ to $\check{\mathcal{R}}$ and $\check{\rho}:[v, P] \mapsto v$ from $\check{\mathcal{R}}$ to the infinite cylinder $\mathcal{C}$. KPZ fluctuations with flat initial condition are expressed in section 2.2 in terms of the trace over $\check{\rho}$ of a holomorphic differential on $\check{\mathcal{R}}$.

The existence of the covering map $\check{\Pi}$ from $\mathcal{R}$ to $\check{\mathcal{R}}$ implies that any loop $\ell_{a, b} \cdot P$ on $\mathcal{R}$ project to a loop on $\check{\mathcal{R}}$. There exists however loops on $\check{\mathcal{R}}$ that may not be obtained in such a way, for instance starting from $\mathbb{C}_{\emptyset}$, the paths $[v-\mathrm{i} \pi, \emptyset] \rightarrow[v+\mathrm{i} \pi, \emptyset]$, Re $v<0$ and $[v, \emptyset] \rightarrow\left[(2 \mathrm{i} \pi m)_{1}, \emptyset\right]=\left[(2 \mathrm{i} \pi m)_{\mathrm{r}}, B_{m}\right] \rightarrow\left[v+2 \mathrm{i} \pi m, B_{m}\right], \operatorname{Re} v>0, m \in \mathbb{Z}$ represented in figure 15 are closed on $\mathcal{\mathcal { R }}$ but not on $\mathcal{R}$.

\subsubsection{Riemann surface $\mathcal{R}^{\Delta}$}

We define in this section Riemann surfaces $\mathcal{R}^{\Delta}, \Delta \sqsubset \mathbb{Z}+1 / 2$ such that the elements $a \in \Delta$ correspond to branch points $2 \mathrm{i} \pi a$ that have been "removed" compared to $\mathcal{R}$.

Let us consider the involutions $D_{a}, a \in \mathbb{Z}+1 / 2$ acting on finite sets $P \sqsubset \mathbb{Z}+1 / 2$ by

$$
D_{a} P=P \ominus\{a\} .
$$

For $\Delta \sqsubset \mathbb{Z}+1 / 2$, we call $G^{\Delta}$ the commutative group generated by the $D_{a}, a \in \Delta$. The orbit of any $P \sqsubset \mathbb{Z}+1 / 2$ under the action of $G^{\Delta}$ is the collection of all sets $Q \sqsubset \mathbb{Z}+1 / 2$ such that $Q \backslash \Delta=P \backslash \Delta$, and orbits can thus be labelled by the sets $P$ such that $P \cap \Delta=\emptyset$.

The group $G^{\Delta}$ acting on sets can be upgraded to a group acting on the Riemann surface $\mathcal{R}$. By the commutativity of symmetric difference, the maps $\mathcal{D}_{a}, a \in \mathbb{Z}+1 / 2$ defined by

$$
\mathcal{D}_{a}[v, P]=\left[v, D_{a} P\right]
$$

are holomorphic automorphisms of $\mathcal{R}$, and deck transformations for the covering map $\Pi$ from $\mathcal{R}$ to the infinite cylinder $\mathcal{C}$. The group $\mathfrak{g}^{\Delta}$ generated by the $\mathcal{D}_{a}, a \in \Delta$ acts properly 

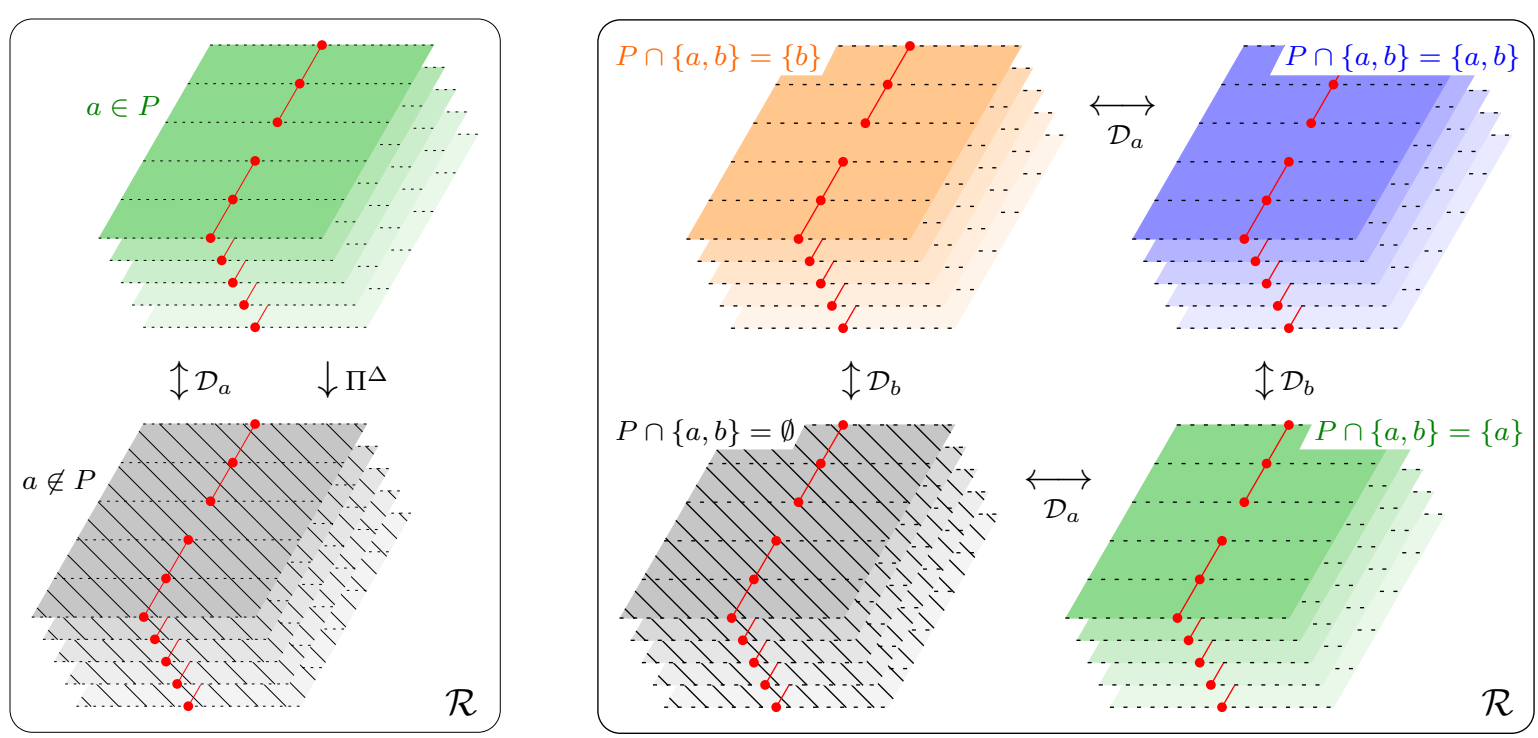

Figure 16: Choice of a fundamental domain of $\mathcal{R}$ under the action of $\mathfrak{g}^{\Delta}$ with $\Delta=\{a\}$ (left) and $\Delta=\{a, b\}$ (right). The sheets $\mathbb{C}_{P}$, partitioned along dashed lines into pairs of half-infinite strips $\mathcal{S}_{P}^{m}=\mathcal{S}_{P}^{\mathrm{l}, m} \cup \mathcal{S}_{P}^{\mathrm{r}, m}$ from 39 , are grouped together according to the value of $P \cap \Delta$. The fundamental domain corresponding to $\mathcal{R}^{\Delta}$ is the hatched portion, made from the sheets $\mathbb{C}_{P}$ with $P \cap \Delta=\emptyset$. How sheets are glued together along branch cuts (red lines, with dots for the branch points) is not represented for clarity.

discontinuously on $\mathcal{R}$, and the quotient $\mathcal{R}^{\Delta}=\mathcal{R} / \mathfrak{g}^{\Delta}$ is a Riemann surface. One has in particular $\mathcal{R}^{\emptyset}=\mathcal{R}$.

Choosing for fundamental domain the collection of sheets $\mathbb{C}_{P}$ with $P \cap \Delta=\emptyset$, the construction above defines the covering map $\Pi^{\Delta}:[v, P] \mapsto[v, P \backslash \Delta]$ from $\mathcal{R}$ to $\mathcal{R}^{\Delta}$, and $\mathcal{R}^{\Delta}$ may be partitioned into sheets $\mathbb{C}_{P}, P \cap \Delta=\emptyset$, images of the sheets of $\mathcal{R}$ by $\Pi^{\Delta}$, see figure 16. The sheet $\mathbb{C}_{P}$ of $\mathcal{R}^{\Delta}$ is glued to the sheet $\mathbb{C}_{P \ominus\left(B_{n} \backslash \Delta\right)}$ along the cut $v \in(2 \mathrm{i} \pi(n-1 / 2), 2 \mathrm{i} \pi(n+1 / 2))$.

The sheets $\mathbb{C}_{P}$ may be further partitioned into pairs of half-infinite strips $\mathcal{S}_{P}^{m}=\mathcal{S}_{P}^{1, m} \cup$ $\mathcal{S}_{P}^{\mathrm{r}, m}, m \in \mathbb{Z}$ with the same notations $(39)$ as in the previous section. Unlike for $\mathcal{R}$, we have not taken a quotient by translations of $2 \mathrm{i} \pi$ here, so that all values $m \in \mathbb{Z}$ must be taken in the partition. This defines the covering map $\rho^{\Delta}:[v, P] \mapsto v-2 \mathrm{i} \pi\left[\frac{\operatorname{Im} v}{2 \pi}\right]$ from $\mathcal{R}^{\Delta}$ to the infinite cylinder $\mathcal{C}$, see figure 1 for a summary of useful covering maps.

\subsubsection{Collection $\overline{\mathcal{R}}$ of Riemann surface $\mathcal{R}^{\Delta}$}

The Riemann surfaces $\mathcal{R}^{\Delta}$ and $\mathcal{R}^{\Delta+1}$ are isomorphic since changing $\Delta$ to $\Delta+1$ amounts to relabelling the sheets. For the application to KPZ in section 2 , it is sometimes convenient, however, to consider sheets from all $\mathcal{R}^{\Delta}$ without the identification $\Delta \equiv \Delta+1$. In order to do this, we introduce bijective operators $\bar{T}_{1 \mid \mathrm{r}}$ acting on pairs of sets $(P, \Delta), P, \Delta \sqsubset \mathbb{Z}+1 / 2$ by $\bar{T}_{1}(P, \Delta)=(P+1, \Delta+1)$ and $\bar{T}_{\mathrm{r}}(P, \Delta)=((P+1) \ominus(\{1 / 2\} \backslash(\Delta+1)), \Delta+1)$. For any $m \in \mathbb{Z}$, the iterated composition of these operators is given by

$$
\begin{aligned}
& \bar{T}_{1}^{m}(P, \Delta)=(P+m, \Delta+m) \\
& \bar{T}_{\mathrm{r}}^{m}(P, \Delta)=\left((P+m) \ominus\left(B_{m} \backslash(\Delta+m)\right), \Delta+m\right) .
\end{aligned}
$$




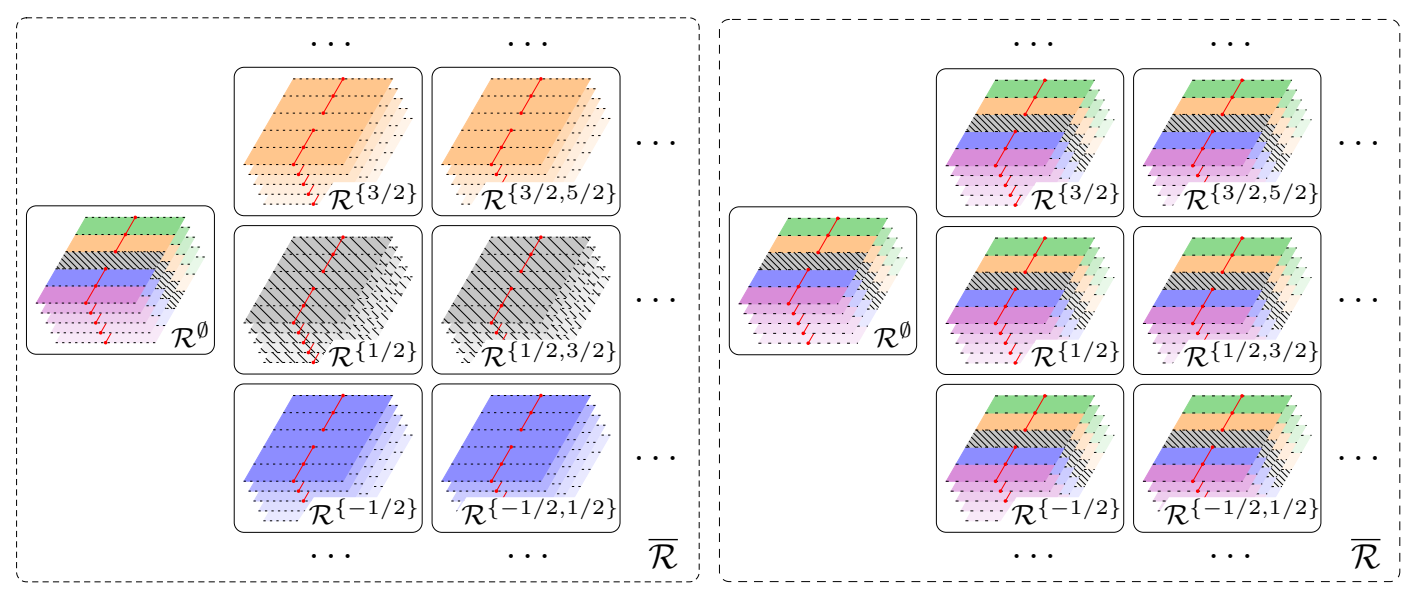

Figure 17: Two choices for the fundamental domain of the collection of Riemann surfaces $\overline{\mathcal{R}}$ under the action of $\overline{\mathfrak{g}}$. The fundamental domain is the hatched portion. How sheets are glued together is not represented for clarity. For the connected component $\mathcal{R}^{\emptyset}=\mathcal{R}$ of $\overline{\mathcal{R}}$, the fundamental domain $\check{\mathcal{R}}$ is chosen as in figure 13 left.

The operators $\bar{T}_{1 \mid \mathrm{r}}$ generate two groups $\bar{G}_{1 \mid \mathrm{r}}=\left\{\bar{T}_{1 \mid \mathrm{r}}^{m}, m \in \mathbb{Z}\right\}$ acting on subsets of $(\mathbb{Z}+$ $1 / 2) \times(\mathbb{Z}+1 / 2)$. The sector $\{(P, \emptyset), P \sqsubset \mathbb{Z}+1 / 2\}$ is an invariant subset under $\bar{G}_{1 \mid \mathrm{r}}$, which essentially reduce to the groups $G_{1 \mid \mathrm{r}}$ of the previous section there, and have in particular the same orbits. Outside of that sector, equivalence classes of pairs of sets in the same orbit under $\bar{G}_{1}$ may be labelled by pairs $(P, \Delta)$ with $\Delta$ arbitrary and $P$ such that its smallest element is equal to e.g. $1 / 2$.

The situation is more complicated for $\bar{G}_{\mathrm{r}}$. Introducing $\lambda_{ \pm}(P, \Delta)=|P|_{ \pm}-|P \ominus \Delta|_{\mp}$, one has for any $P, \Delta \sqsubset \mathbb{Z}+1 / 2$ the identity

$$
\left(\lambda_{ \pm} \circ \bar{T}_{\mathrm{r}}^{m}\right)(P, \Delta)=\lambda_{ \pm}(P, \Delta) \pm m
$$

which implies in particular that $|P|-|P \ominus \Delta|$ is invariant by $\bar{T}_{\mathrm{r}}^{m}$. The equivalence classes of pairs of sets $(P, \Delta)$ in the same orbit under $\bar{G}_{\mathrm{r}}$ may thus be labelled by pairs $(P, \Delta)$ with $|P|_{+}=|P \ominus \Delta|_{-}$. In the sector $|P|=|P \ominus \Delta|$, such pairs verify both $|P|_{ \pm}=|P \ominus \Delta|_{\mp}$.

We consider the collection $\overline{\mathcal{R}}$ of all Riemann surfaces $\mathcal{R}^{\Delta}, \Delta \sqsubset \mathbb{Z}+1 / 2$ and write $[v,(P, \Delta)] \in \overline{\mathcal{R}}$ for the point $[v, P] \in \mathcal{R}^{\Delta}$. The map $\overline{\mathcal{T}}$ whose iterated composition is given by

$$
\overline{\mathcal{T}}^{m}[v,(P, \Delta)]= \begin{cases}{\left[v+2 \mathrm{i} \pi m, \bar{T}_{1}^{m}(P, \Delta)\right]} & \operatorname{Re} v<0 \\ {\left[v+2 \mathrm{i} \pi m, \bar{T}_{\mathrm{r}}^{m}(P, \Delta)\right]} & \operatorname{Re} v>0\end{cases}
$$

with $\bar{T}_{1 \mid \mathrm{r}}^{m}$ defined in 42 is a holomorphic automorphism of $\overline{\mathcal{R}}$ generating a group $\overline{\mathfrak{g}}$. The map $\overline{\mathcal{T}}^{m}$ restricts to an isomorphism between $\mathcal{R}^{\Delta}$ and $\mathcal{R}^{\Delta+m}$ for any $\Delta \sqsubset \mathbb{Z}+1 / 2$. In particular, $\overline{\mathcal{T}}$ has the same action on $\mathcal{R}^{\emptyset}=\mathcal{R}$ as $\mathcal{T}$ defined in (38).

The quotient $\overline{\mathcal{R}} / \overline{\mathfrak{g}}$ corresponds to the collection of Riemann surfaces containing $\check{\mathcal{R}}$ and a representative $\mathcal{R}^{\Delta}$ from each equivalence class $\Delta \equiv \Delta+1$ under isomorphism, see figure 17 left. Another choice of fundamental domain for the action of $\overline{\mathcal{T}}$ consists in taking $\check{\mathcal{R}}$ plus the infinite strips $\mathcal{S}_{P}^{0}, P \cap \Delta=\emptyset$ from all $\mathcal{R}^{\Delta}$ without the identification $\Delta \equiv \Delta+1$, see figure 17 right. 


\section{Functions on the Riemann surfaces $\mathcal{R}, \check{\mathcal{R}}, \mathcal{R}^{\Delta}$}

In this section, we study polylogarithms with half-integer index and several functions built from them living on the Riemann surfaces $\mathcal{R}, \check{\mathcal{R}}, \mathcal{R}^{\Delta}$ defined in section 3.8, and used for KPZ fluctuations in section 2, The results presented until section 4.6 are not new and merely serve to introduce notations. The explicit analytic continuations performed from section 4.7 on the specific functions needed for KPZ are presumably new.

\section{1 $2 \mathrm{i} \pi(\mathbb{Z}+1 / 2)$-continuable functions, translation and analytic continu- ations}

Throughout section 4, we consider functions analytic in the domain

$$
\mathbb{D}=\mathbb{C} \backslash((-\mathrm{i} \infty,-\mathrm{i} \pi] \cup[\mathrm{i} \pi, \mathrm{i} \infty)),
$$

and which may be continued analytically along any path in $\mathbb{C}$ avoiding the points in $2 \mathrm{i} \pi(\mathbb{Z}+1 / 2)$, i.e. such paths never encounter branch points, poles or essential singularities. We borrow the terminology $2 \mathrm{i} \pi(\mathbb{Z}+1 / 2)$-continuable from the theory of resurgent function for this class of functions. In the presence of branch points, which must necessarily belong to $2 \mathrm{i} \pi(\mathbb{Z}+1 / 2)$, new functions analytic in $\mathbb{D}$ are generated by crossing branch cuts.

We introduce the notation $A_{n}^{1} f$ (respectively $A_{n}^{\mathrm{r}} f$ ) for the function obtained from a $2 \mathrm{i} \pi(\mathbb{Z}+1 / 2)$-continuable function $f$ after crossing the (potential) branch cut ( $2 \mathrm{i} \pi(n-$ $1 / 2), 2 \mathrm{i} \pi(n+1 / 2)), n \in \mathbb{Z}$ from left to right (resp. from right to left), i.e. when the function $f$ is continued analytically along the path $x+2 \mathrm{i} \pi n$ with $x$ increasing from $0^{-}$to $0^{+}$(resp. decreasing from $0^{+}$to $0^{-}$), see figure 18 right. Both $A_{n}^{1 / \mathrm{r}} f$ are understood as analytic functions in $\mathbb{D}$. When the analytic continuation from both sides gives the same result, which happens if the branch points of $f$ are of square root type, we write $A_{n}$ instead of $A_{n}^{\mathrm{l}}$ or $A_{n}^{\mathrm{r}}$. Since $f$ is assumed to be analytic in $\mathbb{D}$, there is no branch cut between $-\mathrm{i} \pi$ and $\mathrm{i} \pi$, and one has $A_{0}^{1 \mid \mathrm{r}} f=f$. Furthermore, the operators $A_{n}^{1}$ and $A_{n}^{\mathrm{r}}$ are inverse of each other, $A_{n}^{\mathrm{l}} A_{n}^{\mathrm{r}} f=A_{n}^{\mathrm{r}} A_{n}^{\mathrm{l}} f=f$.

We will be interested in the following in the interplay between analytic continuation and translation by integer multiples of $2 \mathrm{i} \pi$. Since we are working with functions analytic in $\mathbb{D}$, one has to distinguish the effect of translations on the left and on the right, as two points apart of $2 \mathrm{i} \pi$ moved from the left side to the right side end up crossing distinct branch cuts. We introduce translation operators $T_{1 \mid \mathrm{r}}$ acting on $2 \mathrm{i} \pi(\mathbb{Z}+1 / 2)$-continuable functions $f$, such that $T_{1} f$ and $T_{\mathrm{r}} f$ are analytic in $\mathbb{D}$ and verify respectively $\left(T_{1} f\right)(v)=f(v+2 \mathrm{i} \pi)$ when $\operatorname{Re} v<0$ and $\left(T_{\mathrm{r}} f\right)(v)=f(v+2 \mathrm{i} \pi)$ when $\operatorname{Re} v>0$, see figure 18 right.

One has for any $m, n \in \mathbb{Z}$ the identities $A_{m+n}^{1}=T_{\mathrm{r}}^{-m} A_{n}^{1} T_{1}^{m}$ and $A_{m+n}^{\mathrm{r}}=T_{1}^{-m} A_{n}^{\mathrm{r}} T_{\mathrm{r}}^{m}$. In particular, since $A_{0}^{1 / \mathrm{r}}$ is the identity operator, we observe that the analytic continuation can be deduced from translations on both sides:

$$
\begin{aligned}
& A_{n}^{1}=T_{\mathrm{r}}^{-n} T_{1}^{n} \\
& A_{n}^{\mathrm{r}}=T_{1}^{-n} T_{\mathrm{r}}^{n} .
\end{aligned}
$$

These identities are used in the following as a convenient way to derive analytic continuations of functions defined as integrals of meromorphic differentials.

\subsection{Polylogarithms}

The polylogarithm of index $s \in \mathbb{C}$ is defined for $|z|<1$ by the series

$$
\operatorname{Li}_{s}(z)=\sum_{k=1}^{\infty} \frac{z^{k}}{k^{s}} \text {. }
$$


When $s$ is a non-positive integer, $s \in-\mathbb{N}, \operatorname{Li}_{s}(z)$ reduces to a rational function of $z$ with a pole at $z=1$. Otherwise, analytic continuation beyond the unit disk allows to extend $\mathrm{Li}_{s}$ to an analytic function in $\mathbb{C} \backslash[1, \infty)$, the principal value of $\mathrm{Li}_{s}$, with a branch point at $z=1$ and a branch cut traditionally chosen to be the real numbers larger than 1 . For $s=1, \operatorname{Li}_{1}(z)=-\log (1-z)$, and the branch point is of logarithmic type. The function $\mathrm{Li}_{1}$ can thus be extended to an analytic function on a Riemann surface built from infinitely many sheets $\mathbb{C}_{k}, k \in \mathbb{Z}$, such that the top part of the cut in $\mathbb{C}_{k}$ is glued to the bottom part of the cut in $\mathbb{C}_{k+1}$.

Analytic continuations in the variable $z$ of $\operatorname{Li}_{s}(z)$ when $s \neq 1$ is more involved [86. Indeed, after analytic continuation from below the cut $[1, \infty)$, the function $\operatorname{Li}_{s}(z)$ becomes $\operatorname{Li}_{s}(z)-\frac{2 \mathrm{i} \pi(\log z)^{s-1}}{\Gamma(s)}$ with $\Gamma$ the Euler gamma function. The power $s-1$ in the extra term leads to the same branch point $z=1$ as $\mathrm{Li}_{s}$, while the logarithm gives an additional branch point at $z=0$, and makes the structure of the Riemann surface more complicated since further analytic continuation must take into account how $(\log z)^{s-1}$ varies across branch cuts.

Because of the extra logarithm obtained from analytic continuation, it is useful to consider instead ${ }^{12}$ the function $\operatorname{Li}_{s}\left(-\mathrm{e}^{v}\right)$. In terms of the variable $v$, this function has an alternative expression as the complete Fermi-Dirac integral

$$
\operatorname{Li}_{s}\left(-\mathrm{e}^{v}\right)=-\frac{1}{\Gamma(s)} \int_{0}^{\infty} \mathrm{d} u \frac{u^{s-1}}{\mathrm{e}^{u-v}+1}
$$

for $\operatorname{Re} s>0$, and in terms of the Hurwitz zeta function $\zeta(s, u)=\sum_{k=0}^{\infty}(u+k)^{-s}$ as

$$
\operatorname{Li}_{s}\left(-\mathrm{e}^{v}\right)=\frac{\Gamma(1-s)}{(2 \pi)^{1-s}}\left(\mathrm{i}^{1-s} \zeta\left(1-s, \frac{1}{2}+\frac{v}{2 \mathrm{i} \pi}\right)+\mathrm{i}^{s-1} \zeta\left(1-s, \frac{1}{2}-\frac{v}{2 \mathrm{i} \pi}\right)\right) .
$$

The function $\operatorname{Li}_{s}\left(-\mathrm{e}^{v}\right)$ has the branch points $2 \mathrm{i} \pi a, a \in \mathbb{Z}+1 / 2$. Crossing the branch cut associated to $2 \mathrm{i} \pi a$ in the anti-clockwise direction relative to the branch point transforms $\mathrm{Li}_{s}\left(-\mathrm{e}^{v}\right)$ into $\mathrm{Li}_{s}\left(-\mathrm{e}^{v}\right)-\frac{2 \mathrm{i} \pi(v-2 \mathrm{i} \pi a)^{s-1}}{\Gamma(s)}$, which has the same branch points as the principal value: the function $v \mapsto \operatorname{Li}_{s}\left(-\mathrm{e}^{v}\right)$ thus belongs to the class of $2 \mathrm{i} \pi(\mathbb{Z}+1 / 2)$-continuable functions defined in the previous section if the branch cut is chosen as $(-\mathrm{i} \infty,-\mathrm{i} \pi] \cup[\mathrm{i} \pi, \mathrm{i} \infty)$.

In the much studied case where $s \geq 2$ is an integer, the extra terms obtained after crossing branch cuts are polynomials in $v$, and are thus inert by analytic continuation: crossing a branch cut twice in the same direction simply adds the same extra term once more. All branch points are thus of logarithmic type. When $s$ is not an integer, the situation becomes more complicated since the extra terms are multiplied by a phase after analytic continuation. After setting notations for square roots with specific branch cuts in the next section, we focus in section 4.4 on the case where $s$ is a half-integer, $s \in \mathbb{Z}+1 / 2$, which is the case of interest for KPZ.

\subsection{Square roots $\kappa_{a}(v)$}

Before considering polylogarithms with half-integer index, we introduce for convenience a square root function $\kappa_{a}, a \in \mathbb{Z}+1 / 2$ with branch point $2 \mathrm{i} \pi a$. We define

$$
\kappa_{a}(v)=\sqrt{4 \mathrm{i} \pi a} \sqrt{1-\frac{v}{2 \mathrm{i} \pi a}}=\sqrt{\operatorname{sgn}(a) \mathrm{i}} \sqrt{|4 \pi a|+\operatorname{sgn}(a) 2 \mathrm{i} v},
$$

with the usual branch cut $\mathbb{R}^{-}=(-\infty, 0]$ for the square roots so that the branch cut of $\kappa_{a}$ is the interval $\operatorname{sgn}(a) 2 \mathrm{i} \pi(|a|, \infty)$. In particular, $\kappa_{a}$ is analytic in the domain $\mathbb{D}$ defined 

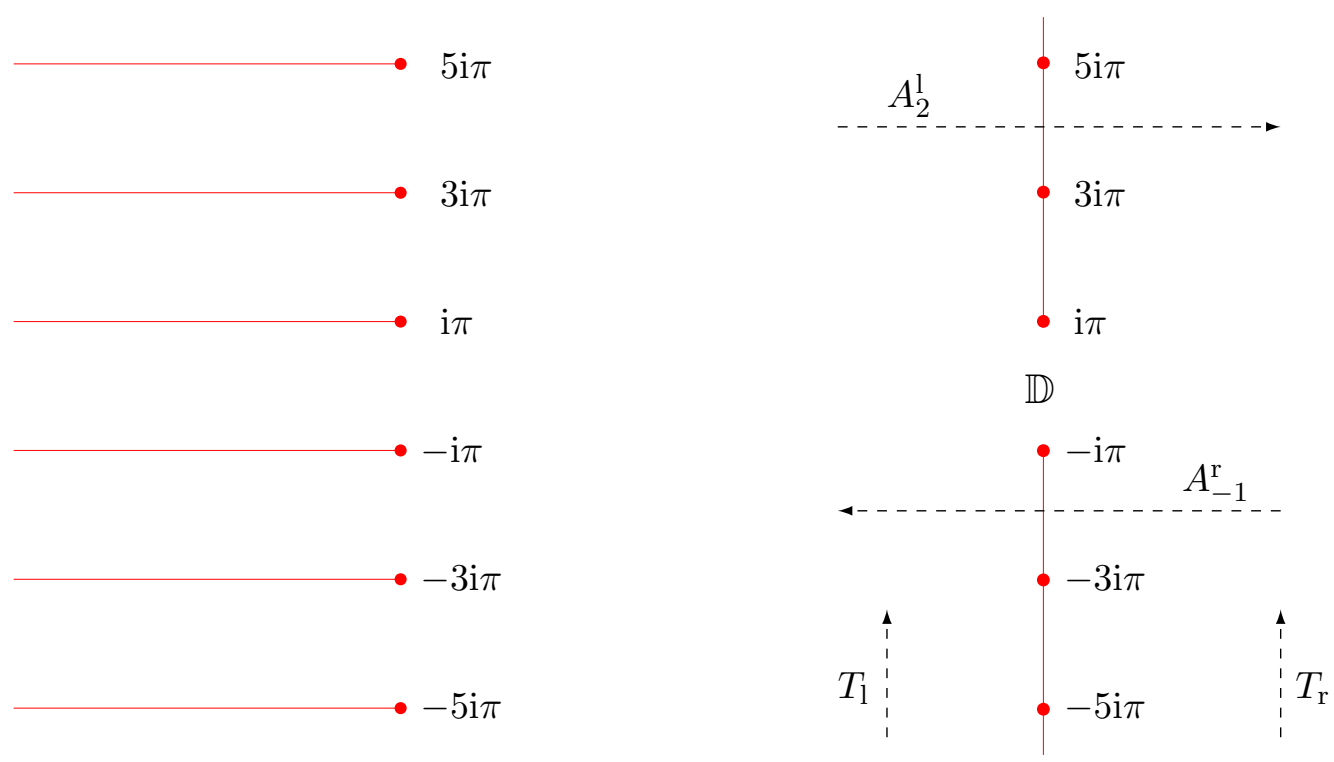

Figure 18: Two possible choices of branch cuts for the function $\chi_{\emptyset}$. The choice on the left, corresponding to the principal value of the polylogarithm $\mathrm{Li}_{5 / 2}$ in $(56)$, is not convenient because of the presence of symmetries $v \mapsto v+2 \mathrm{i} \pi$. The choice on the right, which is the one actually used in the paper, defines by removing the cuts a space $\mathbb{D}=\mathbb{C} \backslash((-\mathrm{i} \infty,-\mathrm{i} \pi] \cup$ $[i \pi, i \infty))$ on which the chosen determination of $\chi_{\emptyset}$ is analytic. Examples of paths of analytic continuation for the translation operators $T_{1}, T_{\mathrm{r}}$ and the analytic continuation operators $A_{n}^{\mathrm{l}}, A_{n}^{\mathrm{r}}, n \in \mathbb{Z}$ defined in section 4.1 are also indicated on the right with dashed arrows.

in (45). The expression (50) for $\kappa_{a}$ reduces to $\kappa_{a}(v)=\sqrt{4 \mathrm{i} \pi a-2 v}$ when $\operatorname{Re} v<0$ and $\kappa_{a}(v)=\operatorname{sgn}(a) \mathrm{i} \sqrt{2 v-4 \mathrm{i} \pi a}$ when $\operatorname{Re} v>0$.

We list a few useful properties of the functions $\kappa_{a}$. The derivative $\kappa_{a}^{\prime}$, also analytic in $\mathbb{D}$, is equal to

$$
\kappa_{a}^{\prime}=-\frac{1}{\kappa_{a}} .
$$

For $v \in \mathbb{D}$, the possible locations of $\kappa_{a}(v)$ in the complex plane are such that both $\log \kappa_{a}$ and $\log \left(\kappa_{a}+\kappa_{b}\right), a, b \in \mathbb{Z}+1 / 2$ are analytic in $\mathbb{D}$ with the branch cut of the logarithm taken as $\mathbb{R}^{-}$, see appendix C.1. Finally, shifting $v$ by an integer multiple of $2 \mathrm{i} \pi$ is equivalent to shifting $a$, up to a possible minus sign: $\kappa_{a}(v+2 \mathrm{i} \pi n)=\kappa_{a-n}(v)$ when $\operatorname{Re} v<0$ and $\kappa_{a}(v+2 \mathrm{i} \pi n)=\sigma_{a}\left(B_{n}\right) \kappa_{a-n}(v)$ when $\operatorname{Re} v>0$, with $\sigma_{a}$ given in (29) and $B_{n}$ in (35). For the logarithm of $\kappa_{a}$, using (180), one has instead $\log \kappa_{a}(v+2 \mathrm{i} \pi n)=\log \kappa_{a-n}(v)$ when $\operatorname{Re} v<0$ and $\log \kappa_{a}(v+2 \mathrm{i} \pi n)=\log \kappa_{a-n}(v)+1_{\left\{a \in B_{n}\right\}} \mathrm{i} \pi \operatorname{sgn}(n)$ when $\operatorname{Re} v>0$. In terms of the translation operators of section 4.1, these identities can be written as

$$
\begin{aligned}
& T_{1}^{n} \kappa_{a}=\kappa_{a-n} \\
& T_{\mathrm{r}}^{n} \kappa_{a}=\sigma_{a}\left(B_{n}\right) \kappa_{a-n} .
\end{aligned}
$$

Using (46), this leads for the analytic continuation from either side of the cut to

$$
A_{n} \kappa_{a}=\sigma_{a}\left(B_{n}\right) \kappa_{a},
$$

which is already obvious from the definition of $\kappa_{a}$. The analytic continuation gives the same result from both sides of the cut since the branch point of $\kappa_{a}$ is of square root type.

\footnotetext{
${ }^{12}$ The minus sign in front of $\mathrm{e}^{v}$ is introduced to make formulas symmetric under complex conjugation.
} 
1090

1091

Similarly, one has for the logarithm of $\kappa_{a}$

$$
\begin{aligned}
& T_{1}^{n} \log \kappa_{a}=\log \kappa_{a-n} \\
& T_{\mathrm{r}}^{n} \log \kappa_{a}=\log \kappa_{a-n}+1_{\left\{a \in B_{n}\right\}} \mathrm{i} \pi \operatorname{sgn}(n),
\end{aligned}
$$

which using (46), leads to distinct analytic continuations from either side,

$$
\begin{aligned}
& A_{n}^{\mathrm{l}} \log \kappa_{a}=\log \kappa_{a}-1_{\left\{a \in B_{n}\right\}} \mathrm{i} \pi \operatorname{sgn}(n) \\
& A_{n}^{\mathrm{r}} \log \kappa_{a}=\log \kappa_{a}+1_{\left\{a \in B_{n}\right\}} \mathrm{i} \pi \operatorname{sgn}(n),
\end{aligned}
$$

and the branch point of $\log \kappa_{a}$ is of logarithmic type.

\subsection{Half-integer polylogarithms and function $\chi$ on $\mathcal{R}$}

We introduce the function

$$
\chi_{\emptyset}(v)=-\frac{\operatorname{Li}_{5 / 2}\left(-\mathrm{e}^{v}\right)}{\sqrt{2 \pi}},
$$

The branch points of $\chi_{\emptyset}$ are the $2 \mathrm{i} \pi a, a \in \mathbb{Z}+1 / 2$. If the principal value of $\mathrm{Li}_{5 / 2}$ is chosen in (56), the branch cut associated to the branch point $a$ is $2 \mathrm{i} \pi a+(-\infty, 0]$, see figure 18 left. We choose instead the branch cut $(-\mathrm{i} \infty,-\mathrm{i} \pi] \cup[\mathrm{i} \pi, \mathrm{i} \infty)$ in the following, see figure $\overline{18}$ right, so that $\chi_{\emptyset}$ is analytic in the domain $\mathbb{D}$ defined in (45). Using $(49)$, this can be done explicitly by writing $\chi_{\emptyset}(v)$ as

$$
\chi_{\emptyset}(v)=\frac{8 \pi^{3 / 2}}{3}\left(\mathrm{e}^{\mathrm{i} \pi / 4} \zeta\left(-\frac{3}{2}, \frac{1}{2}+\frac{v}{2 \mathrm{i} \pi}\right)+\mathrm{e}^{-\mathrm{i} \pi / 4} \zeta\left(-\frac{3}{2}, \frac{1}{2}-\frac{v}{2 \mathrm{i} \pi}\right)\right),
$$

which is indeed analytic for $v \in \mathbb{D}$ if the usual branch cut $\mathbb{R}^{-}$is chosen for the Hurwitz $\zeta$ function $\zeta(-3 / 2, \cdot)$.

From (47), the polylogarithm expression (56) gives for large $|v|$, Re $v<0$ the convergent expansion

$$
\chi_{\emptyset}(v) \simeq-\frac{1}{\sqrt{2 \pi}} \sum_{j=1}^{\infty} \frac{(-1)^{j} \mathrm{e}^{j v}}{j^{5 / 2}} \simeq \frac{\mathrm{e}^{v}}{\sqrt{2 \pi}} .
$$

Using the asymptotic expansion for the Hurwitz zeta function in terms of Bernoulli numbers $B_{r}$,

$$
\zeta(s, u) \simeq-\frac{1}{1-s} \sum_{r=0}^{\infty}\left(\begin{array}{c}
1-s \\
r
\end{array}\right) \frac{B_{r}}{u^{r+s-1}}
$$

when $|u| \rightarrow \infty$ away from the negative real axis $\mathbb{R}^{-}$, the expression 57 for $\chi_{\emptyset}$ gives the asymptotic expansion on the other side of the branch cut,

$$
\chi_{\emptyset}(v) \simeq \frac{32 \pi^{3 / 2}}{15} \sum_{r=0}^{\infty}\left(\begin{array}{c}
5 / 2 \\
2 r
\end{array}\right) \frac{(-1)^{r}\left(2^{1-2 r}-1\right) B_{2 r}}{\left(\frac{v}{2 \pi}\right)^{2 r-5 / 2}} \simeq \frac{(2 v)^{5 / 2}}{15 \pi}+\frac{\pi \sqrt{2 v}}{6}-\frac{7 \pi^{3}}{360(2 v)^{3 / 2}}
$$

when $|v| \rightarrow \infty, \operatorname{Re} v>0$. The function $\chi_{\emptyset}$ has thus an essential singularity at infinity: on the left side of the cut, the convergent expansion (58) for $\chi_{\emptyset}(v)$ is given as a series in $\mathrm{e}^{v}$, while on the right side of the cut, the expansion $(\overline{60})$ of $\chi_{\emptyset}(v)$ has a vanishing radius of convergence in the variable $1 / v$.

From the Hurwitz zeta representation (57), it is possible to rewrite $\chi_{\emptyset}$ as an infinite sum of powers $3 / 2$. In terms of the square root functions $\kappa_{a}$ defined in (50), the identity 
1114 1115

$\zeta(s, u+1)=\zeta(s, u)-u^{-s}$, valid for any $s \in \mathbb{C} \backslash\{1\}$, even though $\zeta(s, u)=\sum_{k=0}^{\infty}(u+k)^{-s}$ only holds when $\operatorname{Re} s>1$, leads for any non-negative integer $M$ to

$$
\begin{aligned}
\chi_{\emptyset}(v)= & \frac{8 \pi^{3 / 2}}{3}\left(\mathrm{e}^{\mathrm{i} \pi / 4} \zeta\left(-\frac{3}{2}, M+\frac{1}{2}+\frac{v}{2 \mathrm{i} \pi}\right)+\mathrm{e}^{-\mathrm{i} \pi / 4} \zeta\left(-\frac{3}{2}, M+\frac{1}{2}-\frac{v}{2 \mathrm{i} \pi}\right)\right) \\
& -\sum_{a=-M+1 / 2}^{M-1 / 2} \frac{\kappa_{a}^{3}(v)}{3},
\end{aligned}
$$

where the sum is over half integers $a \in \mathbb{Z}+1 / 2$ between $-M+1 / 2$ and $M-1 / 2$. In the expression above, the first term containing the $\zeta$ functions is analytic in the strip $-(M+1 / 2) \pi<\operatorname{Re} v<(M+1 / 2) \pi$ : the only branch points in the strip are contributed by the sum. Our choice of branch cuts for the functions $\kappa_{a}$ and $\zeta(-3 / 2, \cdot)$ then agrees with the requirement that the expression (61) must be analytic in $\mathbb{D}$.

Taking $M \rightarrow \infty$ and using the asymptotic expansion (59), we finally obtain $\chi_{\emptyset}$ as

$$
\begin{aligned}
\chi_{\emptyset}(v)=\lim _{M \rightarrow \infty}(- & \frac{4(2 \pi M)^{5 / 2}}{15 \pi}-\frac{2 v(2 \pi M)^{3 / 2}}{3 \pi} \\
& \left.+\frac{\left(\pi^{2}+3 v^{2}\right) \sqrt{2 \pi M}}{6 \pi}-\sum_{a=-M+1 / 2}^{M-1 / 2} \frac{\kappa_{a}^{3}(v)}{3}\right) .
\end{aligned}
$$

In this expression, each term of the sum is analytic for $v \in \mathbb{D}$ with our choice of branch cut for the functions $\kappa_{a}$. The two choices of branch cuts in figure 18 are thus analogous to the ones in figure 5 for the finite sum of $m$ square roots defined in (28).

Analytic continuation of $\chi_{\emptyset}$ across the branch cut $(2 \mathrm{i} \pi(n-1 / 2), 2 \mathrm{i} \pi(n+1 / 2))$ changes the signs of a finite number of terms in the infinite sum representation (62). After a finite number of branch cut crossings, the function $\chi_{\emptyset}$ is replaced by

$$
\begin{aligned}
\chi_{P}(v)=\lim _{M \rightarrow \infty}\left(-\frac{4(2 \pi M)^{5 / 2}}{15 \pi}-\frac{2 v(2 \pi M)^{3 / 2}}{3 \pi}\right. & +\frac{\left(\pi^{2}+3 v^{2}\right) \sqrt{2 \pi M}}{6 \pi} \\
& \left.-\sum_{a=-M+1 / 2}^{M-1 / 2} \sigma_{a}(P) \frac{\kappa_{a}^{3}(v)}{3}\right),
\end{aligned}
$$

$P \sqsubset \mathbb{Z}+1 / 2$, where the sign $\sigma_{a}(P)$ is defined in $(29)$. The set $P$ contains the indices $a \in \mathbb{Z}+1 / 2$ for which the sign of $\kappa_{a}^{3}(v)$ has been flipped an odd number of times after crossing branch cuts, and depends on the path along which the analytic continuation is taken. When $P$ is the empty set $\emptyset, \chi_{P}$ reduces to $\chi_{\emptyset}$. The difference between $\chi_{P}$ and $\chi_{\emptyset}$ is a finite sum,

$$
\chi_{P}(v)=\chi_{\emptyset}(v)+\sum_{a \in P} \frac{2 \kappa_{a}^{3}(v)}{3} .
$$

The expressions $(63)$ and $(64)$ for $\chi_{P}$ are manifestly analytic in $\mathbb{D}$ with our choice of branch cuts for $\chi_{\emptyset}$ and $\kappa_{a}$.

In terms of the operators $A_{n}$ defined in section 4.1 , analytic continuations across branch cuts of $\chi_{P}$ are simply given by

$$
A_{n} \chi_{P}=\chi_{P \ominus B_{n}},
$$

where the symmetric difference operator $\ominus$ and the set $B_{n}$ are defined in (30) and 35. Since all the branch cuts of $\chi_{P}$ are of square root type, the analytic continuations are independent of the side from which the branch cut is crossed. 
The functions $\chi_{P}$ on $\mathbb{D}$ define a function $\chi$ analytic ${ }^{13}$ on the Riemann surface $\mathcal{R}$ of section 3.8.1 by

$$
\chi([v, P])=\chi_{P}(v) .
$$

Derivatives of $\chi_{\emptyset}$ can also be extended to functions on $\mathcal{R}$. The function $\chi^{\prime}$, defined by $\chi^{\prime}([v, P])=\chi_{P}^{\prime}(v)$ is still analytic on $\mathcal{R}$. The function $\chi^{\prime \prime}$, defined by $\chi^{\prime \prime}([v, P])=\chi_{P}^{\prime \prime}(v)$ is only meromorphic on $\mathcal{R}$, as it has poles at the points $\left[(2 \mathrm{i} \pi a)_{1 \mid \mathrm{r}}, P\right]$.

\subsection{Symmetries of $\chi$}

The expansion $\operatorname{Li}_{s}(z)=\sum_{k=1}^{\infty} z^{k} / k^{s}$, valid for $|z|<1$, indicates that $\chi_{\emptyset}(v)$ is periodic with period $2 \mathrm{i} \pi$ in the sector $\operatorname{Re} v<0$. This is no longer true when $\operatorname{Re} v>0$ since the points $v$ and $v+2 \mathrm{i} \pi$ end up in distinct sheets of $\mathcal{R}$ when moved continuously from $\operatorname{Re} v<0$, which leads to more complicated symmetries.

The action of translations on $\chi_{P}$ can be deduced from its expression (63) as an infinite sum. Recalling the translation operators $T_{1 \mid \mathrm{r}}$ from section 4.1 and using $\kappa_{a}(v) \simeq \sqrt{4 \mathrm{i} \pi a}-$ $v / \sqrt{4 \mathrm{i} \pi a}$ when $|a| \rightarrow \infty$, the identities $(52$ lead to

$$
\begin{aligned}
& T_{1}^{-n} \chi_{P}=\chi_{P+n} \\
& T_{\mathrm{r}}^{-n} \chi_{P}=\chi_{(P+n) \ominus B_{n}}
\end{aligned}
$$

for any $n \in \mathbb{Z}$, with $B_{n}$ defined in (35). More explicitly, $\chi_{P}(v-2 \mathrm{i} \pi n)=\chi_{P+n}(v)$ when $\operatorname{Re} v<0$ and $\chi_{P}(v-2 \mathrm{i} \pi n)=\chi_{(P+n) \ominus B_{n}}(v)$ when $\operatorname{Re} v>0$. The identities (67) are compatible with analytic continuation (65) through (46) since $\left(P \ominus B_{n}\right)-n=(P-n) \ominus B_{-n}$.

In terms of the map $\mathcal{T}$ defined in (38), the identities (67) correspond to the symmetry $\chi \circ \mathcal{T}=\chi$ for the function $\chi$ on $\mathcal{R}$. Since the Riemann surface $\check{\mathcal{R}}$ is defined as the quotient of $\mathcal{R}$ by the group generated by $\mathcal{T}$, this means that $\chi$ may also be defined as an analytic function on $\mathcal{R}$. In the following, we use the same notation $\chi$ for both the function defined on $\mathcal{R}$ and on $\check{\mathcal{R}}$, and similarly for the functions $\chi^{\prime}$ and $\chi^{\prime \prime}$ built from the derivatives $\chi_{P}^{\prime}$ and $\chi_{P}^{\prime \prime}$.

\subsection{Function $I_{0}$}

We consider for $\nu \in \mathbb{D}$ the function

$$
I_{0}(\nu)=-\frac{1}{4} \int_{-\infty}^{\nu} \frac{\mathrm{d} v}{1+\mathrm{e}^{-v}}=-\frac{1}{4} \int_{-\infty}^{\nu} \mathrm{d} v\left(\frac{1}{2}+\sum_{a \in \mathbb{Z}+1 / 2} \frac{1}{v-2 \mathrm{i} \pi a}\right)
$$

with a path of integration contained in $\mathbb{D}$, see figure 19 . Since the integrand is analytic in $\mathbb{D}, I_{0}$ is independent from the path of integration. Because of the poles located at $2 \mathrm{i} \pi a$, $a \in \mathbb{Z}+1 / 2$, however, the function $I_{0}$ is defined with the branch cut $(-\mathrm{i} \infty,-\mathrm{i} \pi] \cup[\mathrm{i} \pi, \mathrm{i} \infty)$. The integral can be performed explicitly, and one has the alternative expression

$$
I_{0}(\nu)=\left\{\begin{array}{ll}
-\frac{1}{4} \log \left(1+\mathrm{e}^{\nu}\right) & \operatorname{Re} \nu<0 \\
-\frac{\nu}{4}-\frac{1}{4} \log \left(1+\mathrm{e}^{-\nu}\right) & \operatorname{Re} \nu>0
\end{array} .\right.
$$

An expression manifestly analytic in $\mathbb{D}$ follows from the reflection formula for the Euler $\Gamma$ function $\Gamma\left(\frac{1}{2}-\mathrm{i} z\right) \Gamma\left(\frac{1}{2}+\mathrm{i} z\right)=\pi / \cosh (\pi z)$,

$$
I_{0}(\nu)=-\frac{\log (2 \pi)}{4}-\frac{\nu}{8}+\frac{1}{4} \log \Gamma\left(\frac{1}{2}-\frac{\nu}{2 \mathrm{i} \pi}\right)+\frac{1}{4} \log \Gamma\left(\frac{1}{2}+\frac{\nu}{2 \mathrm{i} \pi}\right) .
$$

\footnotetext{
${ }^{13}$ We recall that the points at infinity, where $\chi_{P}$ has an essential singularity, are understood as punctures and do not belong to $\mathcal{R}$.
} 
Here, $\log \Gamma(z)$ is the principal value of the $\log \Gamma$ function ${ }^{14}$, which is analytic for $z \in \mathbb{C} \backslash \mathbb{R}^{-}$. Alternatively, the $\log \Gamma$ function may be written as an infinite sum of logarithms, and one has

$$
I_{0}(\nu)=-\frac{\log 2}{4}-\frac{\nu}{8}-\frac{1}{4} \sum_{a \in \mathbb{Z}+1 / 2} \log \left(1-\frac{\nu}{2 \mathrm{i} \pi a}\right) .
$$

When $\operatorname{Re} \nu<0$, the function $I_{0}$ verifies from (69) the identity $I_{0}(\nu+2 \mathrm{i} \pi n)=I_{0}(\nu)$ for $n \in \mathbb{Z}$. When $\operatorname{Re} \nu>0$, one has instead $I_{0}(\nu+2 \mathrm{i} \pi n)=I_{0}(\nu)-\mathrm{i} \pi n / 2$. In terms of the translation operators defined in section 4.1, one can write

$$
\begin{aligned}
& T_{1}^{n} I_{0}=I_{0} \\
& T_{\mathrm{r}}^{n} I_{0}=I_{0}-\mathrm{i} \pi n / 2 .
\end{aligned}
$$

The function $I_{0}$ is $2 \mathrm{i} \pi(\mathbb{Z}+1 / 2)$-continuable, as defined in section 4.1. Analytic continuations across the branch cut can then be written solely in terms of the translation operators on both sides as (46), and we obtain

$$
\begin{aligned}
& A_{n}^{\mathrm{l}} I_{0}=I_{0}+\mathrm{i} \pi n / 2 \\
& A_{n}^{\mathrm{r}} I_{0}=I_{0}-\mathrm{i} \pi n / 2,
\end{aligned}
$$

which can also be proved more directly from e.g. 69 .

We note that (73) implies that the domain of definition of the function $I_{0}$ may not be extended to the Riemann surfaces $\mathcal{R}$ or $\check{\mathcal{R}}$. This is a consequence of the presence of logarithmic branch points, coming from the integration of poles, instead of the square root branch points required for $\mathcal{R}$. The domain of definition of the function $\mathrm{e}^{2 I_{0}}$, studied below in section 4.8 , can on the other hand be extended to both $\mathcal{R}$ and $\check{\mathcal{R}}$.

\subsection{Functions $J_{P}$}

We consider for $\nu \in \mathbb{D}$ and $P \sqsubset \mathbb{Z}+1 / 2$ the function

$$
J_{P}(\nu)=\frac{1}{2} f_{-\infty}^{\nu} \mathrm{d} v \chi_{P}^{\prime \prime}(v)^{2}=\lim _{\Lambda \rightarrow \infty}\left(-|P|^{2} \log \Lambda+\frac{1}{2} \int_{-\Lambda}^{\nu} \mathrm{d} v \chi_{P}^{\prime \prime}(v)^{2}\right),
$$

with $\chi_{P}$ given in (64), (57) and a path of integration contained in $\mathbb{D}$, see figure 19 . The regularized integral $f$ is defined for convenience by subtracting the divergent logarithmic term at $-\infty$ coming from (64), (58), with $|P|$ the cardinal of $P$. The integrand in (74) is analytic in $\mathbb{D}$, and $J_{P}$ is independent from the path of integration. The function $J_{P}$ has logarithmic singularities at $2 \mathrm{i} \pi a, a \in \mathbb{Z}+1 / 2$ since $\chi_{P}^{\prime \prime}(v)^{2} \mathrm{~d} v$ is a meromorphic differential of the third kind with simple poles at the $2 \mathrm{i} \pi a$. Additionally, $J_{\emptyset}$ has the large $|\nu|$ asymptotics $J_{\emptyset}(\nu) \simeq \frac{\mathrm{e}^{2 \nu}}{4 \pi}$ when $\operatorname{Re} \nu<0$ and $J_{\emptyset}(\nu) \simeq \frac{\nu^{2}}{\pi^{2}}-\frac{\log \nu}{6}$ when $\operatorname{Re} \nu>0$.

From (67), the function $J_{P}$ transforms under translations of $2 \mathrm{i} \pi$ as $J_{P}(\nu-2 \mathrm{i} \pi n)=$ $J_{P+n}(\nu)$ when $\operatorname{Re} \nu<0$, since the path of integration may be chosen such that $\operatorname{Re} v<0$ everywhere along the path. The situation is more complicated on the other side. Shifting the integration variable by $2 \mathrm{i} \pi n$ in $(74)$, one has $J_{P}(\nu-2 \mathrm{i} \pi n)=\frac{1}{2} f_{-\infty}^{\nu} \mathrm{d} v \chi_{P}^{\prime \prime}(v-2 \mathrm{i} \pi n)^{2}$, where the path of integration crosses the maginary axis in the interval $(2 \mathrm{i} \pi(n-1 / 2), 2 \mathrm{i} \pi(n+1 / 2))$. Introducing $\epsilon>0, \epsilon \rightarrow 0$, the path of integration can be split into a path from $-\infty$ to $2 \mathrm{i} \pi n-$ $\epsilon$ with $\operatorname{Re} v<0$ plus a path from $2 \mathrm{i} \pi n+\epsilon$ to $\nu$ with $\operatorname{Re} v>0$. The translation identities 67 for $\chi_{P}$ then imply $J_{P}(\nu-2 \mathrm{i} \pi n)=\frac{1}{2} f_{-\infty}^{2 \mathrm{i} \pi n-\epsilon} \mathrm{d} v \chi_{P+n}^{\prime \prime}(v)^{2}+\frac{1}{2} \int_{2 \mathrm{i} \pi n+\epsilon}^{\nu} \mathrm{d} v \chi_{(P+n) \ominus B_{n}}^{\prime \prime}(v)^{2}$. Completing the second term by adding the integral on a path from $-\infty$ to $2 \mathrm{i} \pi n+\epsilon$ crossing the imaginary axis between $-\mathrm{i} \pi$ and $\mathrm{i} \pi$, we arrive according to 65 at the integral of the

\footnotetext{
${ }^{14}$ And not merely the logarithm of the $\Gamma$ function for some choice of branch cut for the logarithm.
} 


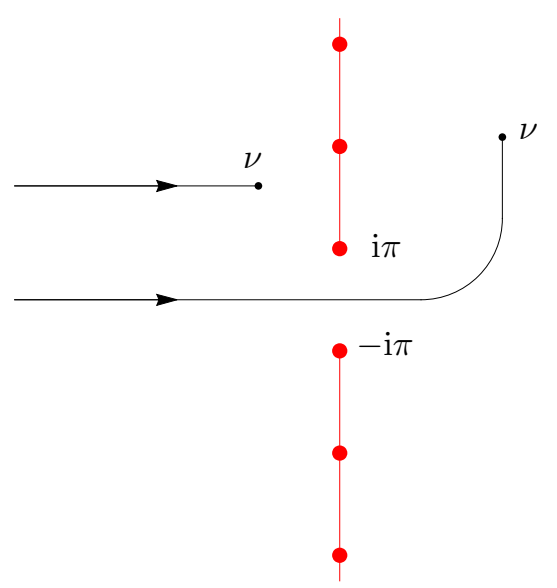

Figure 19: Examples of paths of integration in $\mathbb{D}$ for $I_{0}(\nu)$ in 68$)$ and for $J_{P}(\nu)$ in 74 , so that the functions are analytic in $\mathbb{D}$. The vertical, red lines represent the branch cuts $\mathbb{C} \backslash \mathbb{D}$. The bigger, red dots are the branch points $2 \mathrm{i} \pi a, a \in \mathbb{Z}+1 / 2$.

- $5 \mathrm{i} \pi$

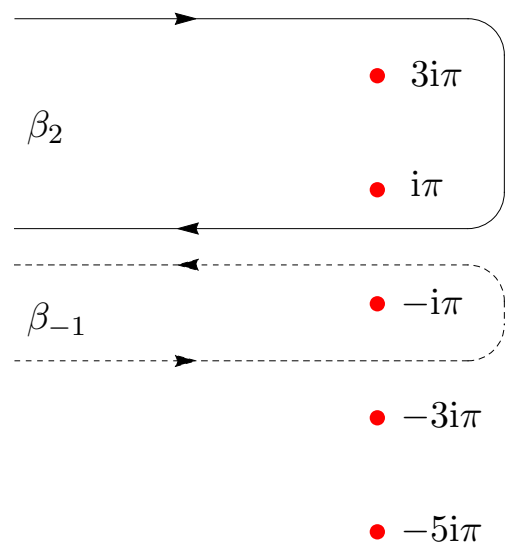

Figure 20: Paths $\beta_{2}$ (solid curve) and $\beta_{-1}$ (dashed curve) from $-\infty$ to $-\infty$ in $\mathbb{C}$.

meromorphic differential $\chi^{\prime \prime}(p)^{2} \mathrm{~d} v$ on a path of the Riemann surface $\mathcal{R}$ (or $\check{\mathcal{R}}$ ), from the puncture $[-\infty, P+n]$ to the puncture $\left[-\infty,(P+n) \ominus B_{n}\right]$, regularized in the usual way at both punctures:

$$
J_{P}(\nu-2 \mathrm{i} \pi n)=J_{(P+n) \ominus B_{n}}(\nu)+\frac{1}{2} f_{\beta_{n} \cdot(P+n)} \chi^{\prime \prime}(p)^{2} \mathrm{~d} v,
$$

with $f_{\beta_{n} \cdot(P+n)} \chi^{\prime \prime}(p)^{2} \mathrm{~d} v=f_{-\infty}^{2 \mathrm{i} \pi n-\epsilon} \mathrm{d} v \chi_{P+n}^{\prime \prime}(v)^{2}-f_{-\infty}^{2 \mathrm{i} \pi n+\epsilon} \mathrm{d} v \chi_{(P+n) \ominus B_{n}}^{\prime \prime}(v)^{2}$. Here, $\beta_{n}$ is a path from $-\infty$ to $-\infty$ encircling the elements of $2 \mathrm{i} \pi B_{n}$ in the clockwise direction if $n>0$ and in the anticlockwise direction if $n<0$, see figure 20, while the path $\beta_{0}$ is empty. The path $\beta_{n} \cdot Q, Q \sqsubset \mathbb{Z}+1 / 2$ lifting $\beta_{n}$ to $\mathcal{R}$ through the covering $[v, P] \mapsto v$ is contained in the sheets $\mathbb{C}_{Q} \cup \mathbb{C}_{Q \ominus B_{n}}$ and links $[-\infty, Q]$ to $\left[-\infty, Q \ominus B_{n}\right]$.

The integral $f_{\beta_{n} \cdot(P+n)} \chi^{\prime \prime}(v)^{2} \mathrm{~d} v$ is computed in appendix $\mathrm{A}$. We find

$$
\frac{1}{2} f_{\beta_{n} \cdot P} \chi^{\prime \prime}(v)^{2} \mathrm{~d} v=W_{P \ominus B_{n}}-W_{P-n}
$$


1213

with

$$
W_{P}=\mathrm{i} \pi\left(|P|_{+}^{2}-|P|_{-}^{2}-\sum_{b \in P} b\right)-2|P| \log 2+\frac{1}{2} \sum_{\substack{b, c \in P \\ b \neq c}} \log \frac{\pi^{2}(b-c)^{2}}{4} .
$$

Here, $|P|_{+}$(respectively $|P|_{-}$) denotes the number of positive (resp. negative) elements of $P$.

The identity (76) leads to $J_{P}(\nu-2 \mathrm{i} \pi n)=J_{(P+n) \ominus B_{n}}(\nu)+W_{(P+n) \ominus B_{n}}-W_{P}$ when $\operatorname{Re} \nu>0$. In terms of the translation operators defined in section 4.1 , one has

$$
\begin{aligned}
& T_{1}^{-n} J_{P}=J_{P+n} \\
& T_{\mathrm{r}}^{-n}\left(J_{P}+W_{P}\right)=J_{(P+n) \ominus B_{n}}+W_{(P+n) \ominus B_{n}} .
\end{aligned}
$$

The function $J_{P}$ has the same branch points $2 \mathrm{i} \pi a, a \in \mathbb{Z}+1 / 2$ as $\chi_{P}$. Analytic continuation across the branch cut $(-\mathrm{i} \infty,-\mathrm{i} \pi) \cup(\mathrm{i} \pi, \mathrm{i} \infty)$ can then be written solely in terms of the translation operators as in 46). Using $P \ominus B_{n}-n=(P-n) \ominus B_{-n}$, we obtain

$$
\begin{aligned}
& A_{n}^{1} J_{P}=J_{P \ominus B_{n}}+W_{P \ominus B_{n}}-W_{P-n} \\
& A_{n}^{\mathrm{r}} J_{P}=J_{P \ominus B_{n}}+W_{P \ominus B_{n}-n}-W_{P} .
\end{aligned}
$$

Thus, $J_{P}$ is related to $J_{P \ominus B_{n}}$ by analytic continuation across $(2 \mathrm{i} \pi(n-1 / 2), 2 \mathrm{i} \pi(n+1 / 2))$, just like the functions $\chi_{P}$ and $\chi_{P \ominus B_{n}}$. The extra terms $W_{P \ominus B_{n}}-W_{P-n}$ and $W_{P \ominus B_{n}-n}-W_{P}$ are however distinct in general, as can be seen from the identity (205) in appendix E, and $J_{P}$ may not be extended to an analytic function on $\mathcal{R}$. Since the difference between the extra terms is from (205) an integer multiple of $i \pi$, it is natural to consider the function $\mathrm{e}^{J_{P}}$ instead. This is done in the next section.

\subsection{Functions $\mathrm{e}^{2 I}, \mathrm{e}^{I+J}$ and $\mathrm{e}^{2 J}$ defined on $\check{\mathcal{R}}$}

We consider in this section functions $\mathrm{e}^{2 I}, \mathrm{e}^{I+J}$ and $\mathrm{e}^{2 J} 15$ built from the functions $I_{0}$ and $J_{P}$ defined respectively in (68) and (74), and which are shown below to be well defined on the Riemann surface $\check{\mathcal{R}}$. These functions are used as building blocks for KPZ fluctuations in section 2 ,

We begin with the function $I_{0}$ from section 4.6 . Equation $(73)$ implies that the analytic continuation of the function $\mathrm{e}^{2 I_{0}}$ across the cut is independent of the side from which the continuation is made, $A_{n} \mathrm{e}^{2 I_{0}}=(-1)^{n} \mathrm{e}^{2 I_{0}}$. It is then possible to extend $\mathrm{e}^{2 I_{0}}$ to a meromorphic function $\mathrm{e}^{2 I}$ on $\mathcal{R}$, defined as

$$
\mathrm{e}^{2 I}([\nu, P])=(-1)^{|P|} \mathrm{e}^{2 I_{0}(\nu)} .
$$

The relations $\left|P \ominus B_{n}\right|=|P|+\left|B_{n}\right|-2\left|P \cap B_{n}\right|$ and $\left|B_{n}\right|=|n|$ indeed imply that the change of sign from $A_{n}$ is equivalent to replacing $P$ by $P \ominus B_{n}$ in 80 . Furthermore, the function $\mathrm{e}^{2 I}$ verifies the symmetry relation $\mathrm{e}^{2 I} \circ \mathcal{T}=\mathrm{e}^{2 I}$ with $\mathcal{T}$ defined in (38), and is thus also well defined on the Riemann surface $\check{\mathcal{R}}$. In fact, since $\mathrm{e}^{2 I_{0}(\nu)}= \pm \sqrt{1+\mathrm{e}^{\nu}}$, the function $\mathrm{e}^{2 I}$ can even be defined on the hyperelliptic-like Riemann surface with branch points $2 \mathrm{i} \pi a, a \in \mathbb{Z}+1 / 2$.

The function $\mathrm{e}^{2 I}$ is meromorphic on $\check{\mathcal{R}}$, and has simple poles at the points $\left[(2 \mathrm{i} \pi a)_{1 \mid \mathrm{r}}, P\right]$, $a \in \mathbb{Z}+1 / 2$ : for $\nu$ close to $2 \mathrm{i} \pi a, \mathrm{e}^{2 I}([\nu, P]) \simeq \pm \mathrm{i} / \sqrt{\nu-2 \mathrm{i} \pi a}$, which corresponds to a pole in the local coordinate $y=\sqrt{\nu-2 \mathrm{i} \pi a}$. The differential defined at $p=[\nu, P] \in \check{\mathcal{R}}$ away

\footnotetext{
${ }^{15}$ We emphasize that only the combination of symbols $\mathrm{e}^{2 I}, \mathrm{e}^{I+J}$ and $\mathrm{e}^{2 J}$ are defined here: $I$ and $J$ alone are not, as there is no meaningful way to extend $I_{0}$ and $J_{P}$ to $\check{\mathcal{R}}$.
} 


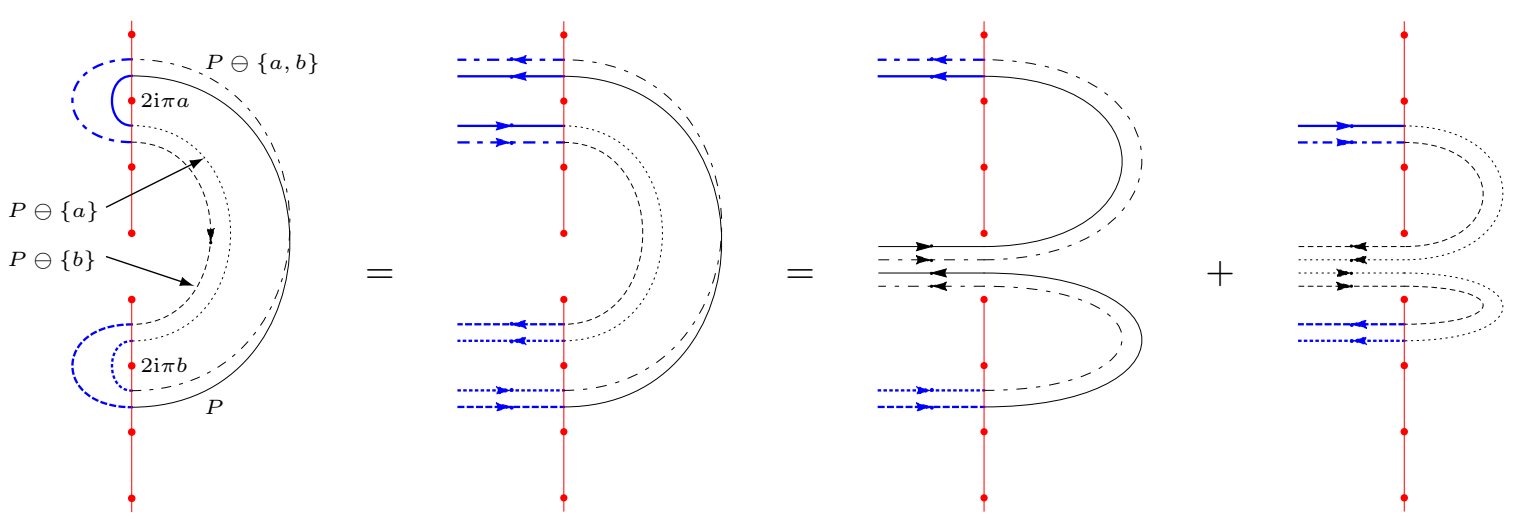

Figure 21: Decomposition of a loop $\ell_{a, b} \cdot P, b<0<a$ in terms of paths of the form $\beta_{n} \cdot Q$. Paths on various sheets $\mathbb{C}_{P}$ are represented differently.

from ramification points by $\mathrm{e}^{2 I}(p) \mathrm{d} \nu$ is on the other hand holomorphic at $\left[(2 \mathrm{i} \pi a)_{1 \mid \mathrm{r}}, P\right]$, $a \in \mathbb{Z}+1 / 2$, since the differential $\mathrm{d} \nu$ becomes proportional to $y \mathrm{~d} y$ in the local coordinate above at $\left[(2 \mathrm{i} \pi a)_{1 \mid \mathrm{r}}, P\right]$ and compensates the pole of the function $\mathrm{e}^{2 I}$.

We now consider the functions $J_{P}$ from section 4.7. Using the identities (207), (211) for the quantities $W_{P}$ and the relation $\left|P \ominus B_{n}\right|=|P|+\sum_{a \in B_{n}} \sigma_{a}(P)$, we obtain after some simplifications

$$
\begin{aligned}
& A_{n}^{1}\left(\frac{(-1)^{|P|} V_{P}^{2}}{4^{|P|}} \mathrm{e}^{J_{P}}\right)=\mathrm{i}^{-n} \frac{(-1)^{\left|P \ominus B_{n}\right|} V_{P \ominus B_{n}}^{2}}{4^{\left|P \ominus B_{n}\right|}} \mathrm{e}^{J_{P \ominus B_{n}}} \\
& A_{n}^{\mathrm{r}}\left(\frac{(-1)^{|P|} V_{P}^{2}}{4^{|P|}} \mathrm{e}^{J_{P}}\right)=\mathrm{i}^{n} \frac{(-1)^{\left|P \ominus B_{n}\right|} V_{P \ominus B_{n}}^{2}}{4^{\left|P \ominus B_{n}\right|}} \mathrm{e}^{J_{P \ominus B_{n}}},
\end{aligned}
$$

where $V_{P}$ is the Vandermonde determinant (5). Comparison with analytic continuations 73 for $I_{0}$ implies that the function $\mathrm{e}^{I+J}$, defined for $\nu \in \mathbb{D}, P \sqsubset \mathbb{Z}+1 / 2$ by

$$
\mathrm{e}^{I+J}([\nu, P])=\frac{(-1)^{|P|} V_{P}^{2}}{4^{|P|}} \mathrm{e}^{I_{0}(\nu)+J_{P}(\nu)},
$$

is well defined on the Riemann surface $\mathcal{R}$. Furthermore, using $(72),(78)$ and $(211)$, the function $\mathrm{e}^{I+J}$ verifies the symmetry $\mathrm{e}^{I+J} \circ \mathcal{T}=\mathrm{e}^{I+J}$, so that $\mathrm{e}^{I+J}$ is also well defined on $\check{\mathcal{R}}$.

The function $\mathrm{e}^{I+J}$ is meromorphic on $\check{\mathcal{R}}$, with the same simple poles as $\mathrm{e}^{2 I}$ at the points $\left[(2 \mathrm{i} \pi a)_{1 \mid \mathrm{r}}, P\right], a \in \mathbb{Z}+1 / 2$. The poles come from logarithmic singularities at the points $v=2 \mathrm{i} \pi a$ in the integral representations $(68)$ and $(74)$ for the functions $I_{0}$ and $J_{P}$. From the infinite sum representation 162 for $\chi_{P}^{\prime \prime}$, the functions $I_{0}$ and $J_{P}$ contribute each half of the logarithmic terms, and one has $\mathrm{e}^{I+J}([\nu, P]) \propto 1 / \sqrt{\nu-2 \mathrm{i} \pi a}$ when $\nu \rightarrow 2 \mathrm{i} \pi a$. The meromorphic differential defined at $p=[\nu, P] \in \mathcal{R}$ away from ramification points by $\mathrm{e}^{I+J}(p) \mathrm{d} \nu$ is holomorphic at $\left[(2 \mathrm{i} \pi a)_{1 \mid \mathrm{r}}, P\right], a \in \mathbb{Z}+1 / 2$ for the same reason as for $\mathrm{e}^{2 I}$.

Finally, the function $\mathrm{e}^{2 J}=\left(\mathrm{e}^{I+J}\right)^{2} / \mathrm{e}^{2 I}$, more explicitly

$$
\mathrm{e}^{2 J}([\nu, P])=(-1)^{|P|} 2^{-4|P|} V_{P}^{4} \mathrm{e}^{2 J_{P}(\nu)},
$$

is also well defined and meromorphic on $\check{\mathcal{R}}$, with simple poles at the points $\left[(2 \mathrm{i} \pi a)_{1 \mid \mathrm{r}}, P\right]$, $a \in \mathbb{Z}+1 / 2$, while the differential $\mathrm{e}^{2 J}(p) \mathrm{d} \nu$ is holomorphic.

The functions $\mathrm{e}^{2 I}, \mathrm{e}^{2 J}$ and $\mathrm{e}^{I+J}$ are defined through $I_{0}(\nu), J_{P}(\nu)$ as integrals on a path contained in $\mathbb{D}$ between $-\infty$ and $\nu$ according to (68), (74). An alternative point 


\footnotetext{
${ }^{16}$ With additional essential singularities for the points at infinity.

${ }^{17}$ The function $v \mapsto-\frac{1}{2} \frac{1}{1+\mathrm{e}^{-v}}$ has residues $-1 / 2$ in $\mathbb{C}$ instead. The distinction with $\varphi$ comes from the fact that the projection of a loop on $\check{\mathcal{R}}$ encircling $\left[(2 \mathrm{i} \pi a)_{1 \mid \mathrm{r}}, P\right]$ to the complex plane by the covering map $[v, P] \mapsto v$ encircles $2 \mathrm{i} \pi a$ an even number of times, see figure 3 . In terms of the local coordinate $y=\sqrt{v-2 \mathrm{i} \pi a}$ at $\left[(2 \mathrm{i} \pi a)_{1 \mid \mathrm{r}}, P\right]$, this is a consequence of $\mathrm{d} v /(v-2 \mathrm{i} \pi a)=2 \mathrm{~d} y / y$.

$y=\sqrt{v-2 i \pi a}$ at $\left./(2 i \pi a){ }_{r}, P\right]$, this is a consequence of $v /(v-2 i \pi a)=2 d y / y$.
}

$$
\begin{aligned}
& \Omega_{2 I}(q)=\varphi(q) \mathrm{d} \nu \\
& \Omega_{2 J}(q)=\chi^{\prime \prime}(q)^{2} \mathrm{~d} \nu \\
& \Omega_{I+J}(q)=\left(\frac{\varphi(q)}{2}+\frac{\chi^{\prime \prime}(q)^{2}}{2}\right) \mathrm{d} \nu,
\end{aligned}
$$

at $q=[\nu, P]$ away from ramification points, one has

$$
\begin{aligned}
& \mathrm{e}^{2 I}(p)=\exp \left(\int_{[-\infty, \emptyset]}^{p} \Omega_{2 I}\right) \\
& \mathrm{e}^{2 J}(p)=\exp \left(\int_{[-\infty, \emptyset]}^{p} \Omega_{2 J}\right) \\
& \mathrm{e}^{I+J}(p)=\exp \left(\int_{[-\infty, \emptyset]}^{p} \Omega_{I+J}\right) .
\end{aligned}
$$

The fact that the functions $\mathrm{e}^{2 I}, \mathrm{e}^{2 J}$ and $\mathrm{e}^{I+J}$ are well defined on $\check{\mathcal{R}}$ imply that the integrals on any loop in $\check{\mathcal{R}}$ of the meromorphic differentials $\Omega_{2 I}, \Omega_{2 J}, \Omega_{I+J}$ are integer multiples of $2 \mathrm{i} \pi$. For small loops around a point $\left[(2 \mathrm{i} \pi a)_{1 \mid \mathrm{r}}, P\right]$, this is a consequence of Cauchy's residue theorem on the Riemann surface, both $\varphi(q) \mathrm{d} \nu$ and $\chi^{\prime \prime}(q)^{2} \mathrm{~d} \nu$ having a residue -1 at that point 17. For non-contractible loops, this is a non-trivial statement. For $\Omega_{2 J}$, it can be checked directly for a given loop by expressing its homology class as a sum of paths of the form $\beta_{n} \cdot P$ from figure 20 and using $(76)$ to compute the integrals. For example, considering the loops $\ell_{a, b} \cdot P$ which generate all loops on $\check{\mathcal{R}}$ that are also closed on $\mathcal{R}$ and are defined in section 3.8.1. see also figure 21, we obtain after some simplifications $\int_{\ell_{a, b}} \Omega_{2 J}=4 \mathrm{i} \pi\left(-1+\sigma_{a}(P) \sigma_{b}(P)\left(\frac{\operatorname{sgn} a-\operatorname{sgn} b}{2}-\operatorname{sgn}(a-b) \frac{1+\operatorname{sgn}(a b)}{2}\right)\right)$, which is an integer multiple of $4 \mathrm{i} \pi$. For loops on $\check{\mathcal{R}}$ corresponding to open paths on $\mathcal{R}$, the integral is generally only an integer multiple of $2 \mathrm{i} \pi$ : for instance, the integral of $\Omega_{2 J}$ on the loop $[v,\{1 / 2\}] \rightarrow\left[(4 \mathrm{i} \pi)_{1},\{1 / 2\}\right]=\left[(4 \mathrm{i} \pi)_{\mathrm{r}},\{3 / 2\}\right] \rightarrow[v+2 \mathrm{i} \pi,\{3 / 2\}], \operatorname{Re} v<0$ is equal to $-2 \mathrm{i} \pi$.

\subsection{Functions on the Riemann surfaces $\mathcal{R}^{\Delta}$}

In this section, we study functions on the Riemann surfaces $\mathcal{R}^{\Delta}$ built in section 3.8.3 by quotienting $\mathcal{R}$ by a group of involutions indexed by the elements of $\Delta \sqsubset \mathbb{Z}+1 / 2$. These functions are used in sections 2.3 and 2.4 for KPZ fluctuations with sharp wedge and stationary initial condition.

\subsubsection{Function $\chi^{\Delta}$}

Let $\Delta \sqsubset \mathbb{Z}+1 / 2$. The covering map $\Pi^{\Delta}:[v, P] \mapsto[v, P \backslash \Delta]$ from $\mathcal{R}$ to $\mathcal{R}^{\Delta}$ allows to define the function $\chi^{\Delta}=2^{-|\Delta|} \operatorname{tr}_{\Pi \Delta} \chi$ analytic on $\mathcal{R}^{\Delta}$, see section 3.4 . More explicitly, for 
any $v \in \mathbb{C}$ and $Q \sqsubset \mathbb{Z}+1 / 2, Q \cap \Delta=\emptyset$, one has $\chi^{\Delta}([v, Q])=2^{-|\Delta|} \sum_{\substack{P \subset \mathbb{Z}+1 / 2 \\ P \backslash \Delta=Q}} \chi_{P}(v)=$ $2^{-|\Delta|} \sum_{A \subset \Delta} \chi_{Q \cup A}(v)$. Using 64), we find

$$
\chi^{\Delta}([v, P])=\chi_{P}^{\Delta}(v),
$$

where $\chi_{P}^{\Delta}$, given by 18

$$
\chi_{P}^{\Delta}(v)=\frac{\chi_{P}(v)+\chi_{P \ominus \Delta}(v)}{2},
$$

generalizes the function $\chi_{P}$ of section 4.5 . The function $\chi_{P}^{\Delta}$ has the infinite sum representation

$$
\begin{aligned}
\chi_{P}^{\Delta}(v)=\lim _{M \rightarrow \infty}\left(-\frac{4(2 \pi M)^{5 / 2}}{15 \pi}-\frac{2 v(2 \pi M)^{3 / 2}}{3 \pi}\right. & +\frac{\left(\pi^{2}+3 v^{2}\right) \sqrt{2 \pi M}}{6 \pi} \\
& \left.-\sum_{a=-M+1 / 2}^{M-1 / 2} 1_{\{a \notin \Delta\}} \sigma_{a}(P) \frac{\kappa_{a}^{3}(v)}{3}\right)
\end{aligned}
$$

with $\sigma_{a}$ defined in $\sqrt{29}$ and $\kappa_{a}$ in $\sqrt{50}$, and verifies $\chi_{P}^{\Delta}=\chi_{P \backslash \Delta}^{\Delta}$.

Since $\chi^{\Delta}$ is analytic on $\mathcal{R}^{\Delta}$, the function $\chi_{P}^{\Delta}$ defined on $\mathbb{D}$ has the analytic continuation

$$
A_{n} \chi_{P}^{\Delta}=\chi_{P}^{\Delta} \ominus\left(B_{n} \backslash \Delta\right)
$$

across branch cuts. Alternatively, (89) can be proved directly from (65) using the general identity $\chi_{P}^{\Delta}=\chi_{P}^{\Delta}{ }_{\ominus A}, A \subset \Delta$ with $A=B_{n} \cap \Delta$. From (67), the functions $\chi_{P}^{\Delta}$ also verify the shift identities

$$
\begin{aligned}
& T_{1}^{-n} \chi_{P}^{\Delta}=\chi_{P+n}^{\Delta+n} \\
& T_{\mathrm{r}}^{-n} \chi_{P}^{\Delta}=\chi_{(P+n) \ominus\left(B_{n} \backslash(\Delta+n)\right)}^{\Delta+n},
\end{aligned}
$$

where we used again $\chi_{P}^{\Delta}=\chi_{P}^{\Delta}{ }_{\ominus A}, A \subset \Delta$ for the second line. In terms of the collection $\overline{\mathcal{R}}$ of all Riemann surfaces $\mathcal{R}^{\Delta}$ of section 3.8.3, of the function $\bar{\chi}([v,(P, \Delta)])=\chi_{P}(v)$ defined on $\overline{\mathcal{R}}$, and of the holomorphic map $\overline{\mathcal{T}}$ on $\mathcal{R}$ defined in $(44)$, the identity $(90)$ simply rewrites as $\bar{\chi} \circ \overline{\mathcal{T}}=\bar{\chi}$.

Finally, we also define functions $\chi^{\prime \Delta}, \chi^{\prime \prime \Delta}$ from derivatives of $\chi_{P}^{\Delta}$ as

$$
\begin{aligned}
& \chi^{\prime \Delta}([v, P])=\chi_{P}^{\prime \Delta}(v) \\
& \chi^{\prime \prime \Delta}([v, P])=\chi_{P}^{\prime \prime \Delta}(v) .
\end{aligned}
$$

The function $\chi^{\prime \Delta}$ is analytic on $\mathcal{R}^{\Delta}$ while $\chi^{\prime \prime \Delta}$ is meromorphic on $\mathcal{R}^{\Delta}$. Both verify the same translation symmetries as $\chi^{\Delta}$.

\subsubsection{Functions $J_{P}^{\Delta}$}

We now consider $J_{P}^{\Delta}$ generalizing the function $J_{P}$ of section 4.7 , defined from the second derivative $\chi_{P}^{\prime \prime \Delta}$ of $\chi_{P}^{\Delta}$ as

$$
\begin{aligned}
J_{P}^{\Delta}(\nu) & =\frac{1}{2} \int_{-\infty}^{\nu} \mathrm{d} v \chi_{P}^{\prime \prime \Delta}(v)^{2} \\
& =\lim _{\Lambda \rightarrow \infty}\left(-\frac{(|P|+|P \ominus \Delta|)^{2}}{4} \log \Lambda+\frac{1}{2} \int_{-\Lambda}^{\nu} \mathrm{d} v \chi_{P}^{\prime \prime \Delta}(v)^{2}\right),
\end{aligned}
$$

\footnotetext{
${ }^{18}$ For later convenience, we define $\chi_{P}^{\Delta}$ in 87$]$ in terms of $P \ominus \Delta$ instead of $P \cup \Delta$ when $P \cap \Delta \neq \emptyset$.
} 
1318

1319

1320

1321

1322

1323

1324

1325

1326

1336

1337

1338

for $\nu \in \mathbb{D}$, with a path of integration contained in $\mathbb{D}$. One has $J_{P}^{\Delta}=J_{P \backslash \Delta}^{\Delta}$.

The same reasoning as in section 4.7 gives for any $n \in \mathbb{Z}$ the identities $J_{P}^{\Delta}(\nu-2 \mathrm{i} \pi n)=$ $J_{P+n}^{\Delta+n}(\nu)$ when $\operatorname{Re} \nu<0$ and $J_{P}^{\Delta}(\nu-2 \mathrm{i} \pi n)=J_{(P+n) \ominus\left(B_{n} \backslash(\Delta+n)\right)}(\nu)+\frac{1}{2} f_{\beta_{n} \cdot(P+n)} \chi^{\prime \prime \Delta+n}(p) \mathrm{d} v$ with $\beta_{n} \cdot(P+n)$ the lift to the sheet $P+n$ of $\mathcal{R}^{\Delta+n}$ of the path in the complex plane $\beta_{n}$ from figure 20. The integral over $\beta_{n} \cdot(P+n)$ can be computed similarly as in appendix A. We obtain eventually

$$
\begin{aligned}
& T_{1}^{-n} J_{P}^{\Delta}=J_{P+n}^{\Delta+n} \\
& T_{\mathrm{r}}^{-n} J_{P}^{\Delta}=J_{(P+n) \ominus\left(B_{n} \backslash(\Delta+n)\right)}^{\Delta+n}+W_{(P+n) \ominus\left(B_{n} \backslash(\Delta+n)\right)}^{\Delta+n}-W_{P}^{\Delta},
\end{aligned}
$$

where

$$
\begin{aligned}
W_{P}^{\Delta}=\frac{\mathrm{i} \pi}{4} & \left(\left(|P|_{+}+|P \ominus \Delta|_{+}\right)^{2}-\left(|P|_{-}+|P \ominus \Delta|_{-}\right)^{2}\right)-\frac{\mathrm{i} \pi}{2}\left(\sum_{b \in P} b+\sum_{b \in P \ominus \Delta} b\right) \\
& -(|P|+|P \ominus \Delta|) \log 2+\frac{1}{4} \sum_{\substack{b, c \in P \\
b \neq c}} \log \frac{\pi^{2}(b-c)^{2}}{4}+\frac{1}{4} \sum_{\substack{b, c \in P \ominus \Delta \\
b \neq c}} \log \frac{\pi^{2}(b-c)^{2}}{4}
\end{aligned}
$$

generalizes the constants $W_{P}=W_{P}^{\emptyset}$ of $(77)$. Using $(46)$, the analytic continuation of $J_{P}^{\Delta}$ across branch cuts is given by

$$
\begin{aligned}
& A_{n}^{1} J_{P}^{\Delta}=J_{P \ominus\left(B_{n} \backslash \Delta\right)}^{\Delta}+W_{P \ominus\left(B_{n} \backslash \Delta\right)}^{\Delta}-W_{P-n}^{\Delta-n} \\
& A_{n}^{\mathrm{r}} J_{P}^{\Delta}=J_{P \ominus\left(B_{n} \backslash \Delta\right)}^{\Delta}+W_{P \ominus\left(B_{n} \backslash \Delta\right)-n}^{\Delta-n}-W_{P}^{\Delta} .
\end{aligned}
$$

As in section 4.7, the analytic continuation from each side of the branch cuts does not give the same result, so that $J_{P}^{\Delta}$ may not be extended to $\mathcal{R}^{\Delta}$. For the application to KPZ in sections 2.3 and 2.4 , however, only $\mathrm{e}^{2 J_{P}^{\Delta}}$ is needed. Using the identity 213 , we find that the function $\mathrm{e}^{2 J^{\Delta}}$ generalizing $\mathrm{e}^{2 J}=\mathrm{e}^{2 J^{\emptyset}}$ and defined by

$$
\mathrm{e}^{2 J^{\Delta}}([\nu, P])=(\mathrm{i} / 4)^{2|P \backslash \Delta|}\left(\prod_{a \in P \backslash \Delta} \prod_{\substack{b \in P \cup \Delta \\ b \neq a}}\left(\frac{2 \mathrm{i} \pi a}{4}-\frac{2 \mathrm{i} \pi b}{4}\right)^{2}\right) \mathrm{e}^{2 J_{P}^{\Delta}(\nu)}
$$

is well defined on $\mathcal{R}^{\Delta}$. The prefactor of $\mathrm{e}^{2 J_{P}^{\Delta}(\nu)}$, which depends on $P$ and $\Delta$ only through $\Delta$ and $P \backslash \Delta$ since $P \cup \Delta=(P \backslash \Delta) \cup \Delta$, has been chosen equal to 1 when $P=\emptyset$. The function $\mathrm{e}^{2 J^{\Delta}}$ can then be expressed alternatively as

$$
\mathrm{e}^{2 J^{\Delta}}(p)=\exp \left(f_{[-\infty, \emptyset]}^{p} \Omega_{2 J}^{\Delta}\right)
$$

independently from the path between $[-\infty, \emptyset]$ and $p$ in $\mathcal{R}^{\Delta}$, with $\Omega_{2 J}^{\Delta}$ the meromorphic differential of the third kind

$$
\Omega_{2 J}^{\Delta}([\nu, P])=\chi^{\prime \prime \Delta}([\nu, P])^{2} \mathrm{~d} \nu .
$$

$$
\mathrm{e}^{2 J^{\Delta}}([\nu-2 \mathrm{i} \pi m, P])=\left\{\begin{array}{ll}
\mathrm{e}^{2 J^{\Delta+m}}([\nu, P+m]) & \operatorname{Re} \nu<0 \\
\mathrm{e}^{2 J^{\Delta+m}}\left(\left[\nu,(P+m) \ominus\left(B_{m} \backslash(\Delta+m)\right)\right]\right) & \operatorname{Re} \nu>0
\end{array} .\right.
$$

Extending $\mathrm{e}^{2 J^{\Delta}}$ to the collection $\overline{\mathcal{R}}$ of all $\mathcal{R}^{\Delta}$ by $\overline{\mathrm{e}^{2 J}}([\nu,(P, \Delta)])=\mathrm{e}^{2 J^{\Delta}}([\nu, P])$, the identity 99 is equivalent to the symmetry $\overline{\mathrm{e}^{2 J}} \circ \overline{\mathcal{T}}=\overline{\mathrm{e}^{2 J}}$ with $\overline{\mathcal{T}}$ defined in 44 . 


\subsection{Functions $K_{P, Q}, K_{P, Q}^{\Delta, \Gamma}$ and $\mathrm{e}^{2 K^{\Delta, \Gamma}}$}

In this section, we study functions on products of Riemann surfaces needed for joint statistics of the KPZ height at multiple times.

\subsubsection{Functions $K_{P, Q}$}

Given two finite sets of half-integers $P$ and $Q$, and $(\nu, \mu)$ in the simply connected domain

$$
\mathbb{D}_{2}=\{(\nu, \mu) \in \mathbb{D} \times \mathbb{D},(\operatorname{Re} \nu \neq \operatorname{Re} \mu) \text { or }(\operatorname{Re} \nu=\operatorname{Re} \mu \text { and } \operatorname{Im}(\nu-\mu) \in(0,2 \pi))\},
$$

we consider the functions

$$
\begin{aligned}
K_{P, Q}(\nu, \mu) & =\frac{1}{2} \int_{-\infty}^{0} \mathrm{~d} u \chi_{P}^{\prime \prime}(u+\nu) \chi_{Q}^{\prime \prime}(u+\mu) \\
& =\lim _{\Lambda \rightarrow \infty}\left(-|P||Q| \log \Lambda+\frac{1}{2} \int_{-\Lambda}^{0} \mathrm{~d} u \chi_{P}^{\prime \prime}(u+\nu) \chi_{Q}^{\prime \prime}(u+\mu)\right) .
\end{aligned}
$$

The path of integration is chosen such that $u+\nu$ and $u+\mu$ both stay in $\mathbb{D}$, which is possible ${ }^{19}$ if $(\nu, \mu) \in \mathbb{D}_{2}$, see figure 22 and $K_{P, Q}$ is thus analytic in $\mathbb{D}_{2}$. When $P=Q$, one recovers $J_{P}(\nu)=\lim _{\mu \rightarrow \nu} K_{P, P}(\nu, \mu)$.

Crossing the branch cuts $\mu, \nu \in(-\mathrm{i} \infty,-\mathrm{i} \pi) \cup(\mathrm{i} \pi, \mathrm{i} \infty), \nu-\mu \in(-\mathrm{i} \infty, 0) \cup(\mathrm{i} \pi, \mathrm{i} \infty)$ produces new functions analytic in $\mathbb{D}_{2}$. In order to obtain explicit formulas for the various analytic continuations of $K_{P, Q}(\nu, \mu)$, it is useful to study first shifts by integer multiples of $2 \mathrm{i} \pi$ in the variables $\nu$ and $\mu$. When both $\operatorname{Re} \nu<0$ and $\operatorname{Re} \mu<0$, the path of integration in 101 can be chosen such that $\operatorname{Re}(u+\nu)<0, \operatorname{Re}(u+\mu)<0$ everywhere, see figure 22 top left, and one has from (67) the identity $K_{P, Q}(\nu-2 \mathrm{i} \pi n, \mu-2 \mathrm{i} \pi m)=$ $K_{P+n, Q+m}(\nu, \mu)$. Similar reasonings leads to $K_{P, Q}(\nu-2 \mathrm{i} \pi n, \mu)=K_{P+n, Q}(\nu, \mu)$ when $\operatorname{Re} \nu<0$ and $K_{P, Q}(\nu, \mu-2 \mathrm{i} \pi m)=K_{P, Q+m}(\nu, \mu)$ when $\operatorname{Re} \mu<0$. Shifting variables with a positive real part is more complicated. Indeed, the argument of the functions $\chi_{P}^{\prime \prime}, \chi_{Q}^{\prime \prime}$ has then a positive real part on some portions of the path of integration in (101) and a negative real part on other portions of the path, so that (67) gives several distinct expressions for the shifts along the path. After some rewriting, one finds in all cases the identity

$$
\begin{aligned}
K_{P, Q}(\nu-2 \mathrm{i} \pi n, \mu-2 \mathrm{i} \pi m)= & K_{\tilde{P}, \tilde{Q}}(\nu, \mu) \\
& +\frac{1}{2} f_{\gamma_{n, m}} \mathrm{~d} u \mathcal{A}_{u}\left(\chi_{P+n}^{\prime \prime}(\cdot+\nu) \chi_{Q+m}^{\prime \prime}(\cdot+\mu)\right)
\end{aligned}
$$

with $\tilde{P}=P+n$ when $\operatorname{Re} \nu<0, \tilde{P}=(P+n) \ominus B_{n}$ when $\operatorname{Re} \nu>0$ and $\tilde{Q}=Q+m$ when $\operatorname{Re} \mu<0, \tilde{Q}=(Q+m) \ominus B_{m}$ when $\operatorname{Re} \mu>0$. We used the notation $\int_{\gamma} \mathrm{d} u \mathcal{A}_{u} f$ for the integral of the analytic continuation of a function $f$ along a path $\gamma$. The path $\gamma_{n, m}$ in the complex plane depends on the real parts of $\nu$ and $\mu$, see figure 23. When $\operatorname{Re} \nu$ and $\operatorname{Re} \nu$ are both negative, the path is empty and the integral in $(102)$ is equal to zero. When $\operatorname{Re} \nu$ and $\operatorname{Re} \nu$ do not have the same sign, the path reduces to $\gamma_{n, m}=\beta_{n}-\nu$ if $\operatorname{Re} \mu<0<\operatorname{Re} \nu$ and $\gamma_{n, m}=\beta_{m}-\mu$ if $\operatorname{Re} \nu<0<\operatorname{Re} \mu$, with $\beta_{n}$ defined in section 4.7, compare with figure 20. Finally, when $\operatorname{Re} \nu$ and $\operatorname{Re} \mu$ are both positive, the path goes from $-\infty$ to $-\infty$ and crosses the lines $\operatorname{Re}(u+\nu)=0$ and $\operatorname{Re}(u+\mu)=0$ along the path at successive points $2 \mathrm{i} \pi n-\nu, 2 \mathrm{i} \pi m-\mu,-\mu,-\nu$ (respectively $2 \mathrm{i} \pi m-\mu, 2 \mathrm{i} \pi n-\nu,-\nu,-\mu)$ if $\operatorname{Re} \nu>\operatorname{Re} \mu$ (resp. $\operatorname{Re} \nu<\operatorname{Re} \mu$ ).

\footnotetext{
${ }^{19}$ At this point, $K_{P, Q}(\nu, \mu)$ is in fact analytic in a domain larger than $\mathbb{D}_{2}$ : in the sector $\operatorname{Re} \nu=\operatorname{Re} \mu$, $\operatorname{Im}(\nu-\mu)$ need not be restricted when the real parts are negative, and one requires only $\operatorname{Im}(\nu-\mu) \in$ $(-2 \pi, 2 \pi)$ when the real parts are positive. Additional terms contributed later on by analytic continuation are however only analytic in $\mathbb{D}_{2}$, and it is thus convenient to add this restriction from the beginning.
} 

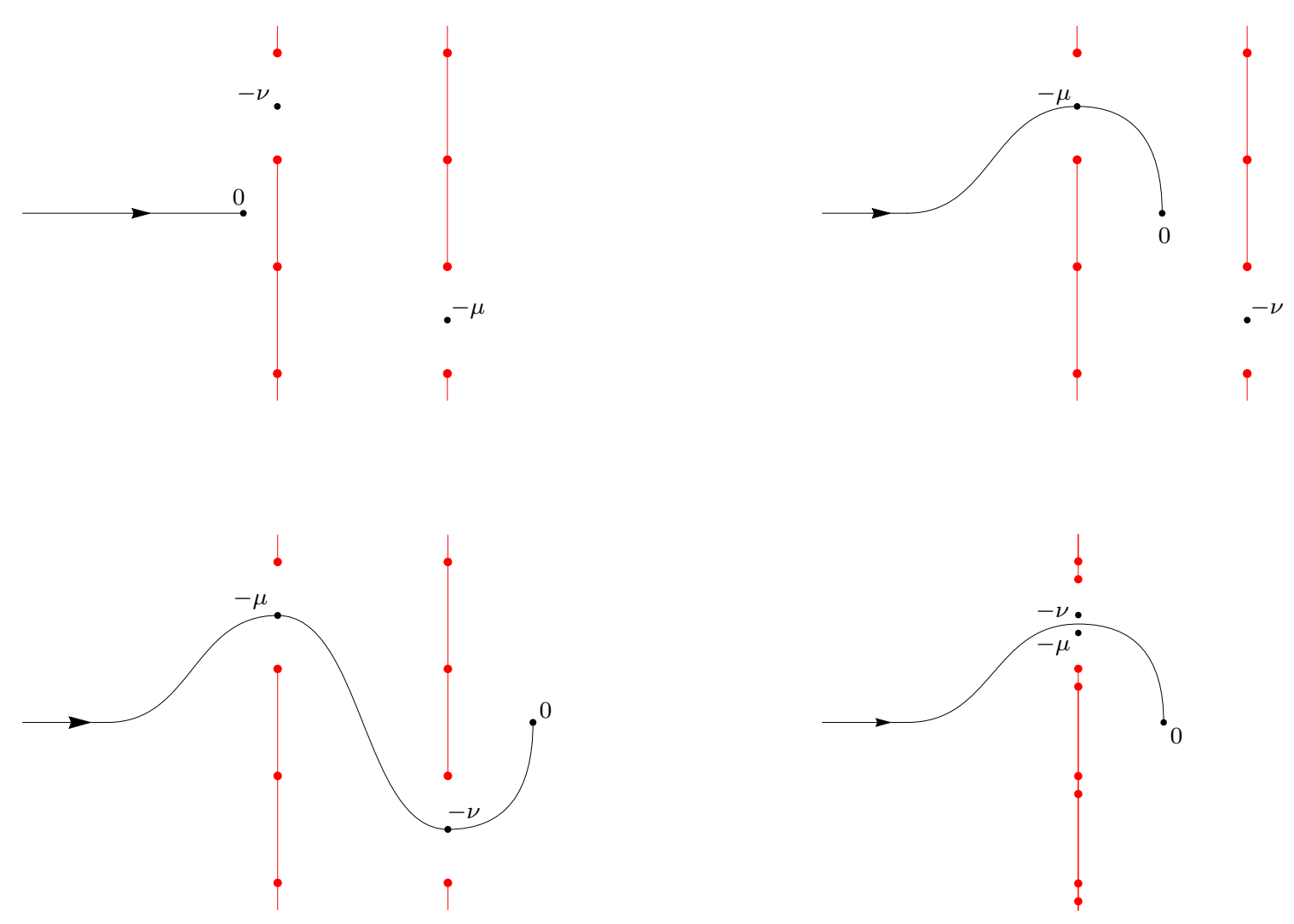

Figure 22: Possible choices for the path of integration in the definition (101) for $K_{P, Q}$. The red, vertical lines are the branch cuts in the variable $u$ of $\chi_{P}^{\prime \prime}(u+\nu)$ and $\chi_{Q}^{\prime \prime}(u+\mu)$, and the red dots on the cuts are the associated branch points $-\nu+2 \mathrm{i} \pi a,-\mu+2 \mathrm{i} \pi a, a \in \mathbb{Z}+1 / 2$. The black curves, ending at $u=0$, represent the path for the variable $u$. From left to right, top to bottom, the four graphs represent respectively the situations $\operatorname{Re} \mu<\operatorname{Re} \nu<0$; $\operatorname{Re} \nu<0<\operatorname{Re} \mu ; \operatorname{Re} \mu>\operatorname{Re} \nu>0 ; \operatorname{Re} \nu=\operatorname{Re} \mu>0$ with $-\pi<\operatorname{Im}(\nu-\mu)<\pi$. 

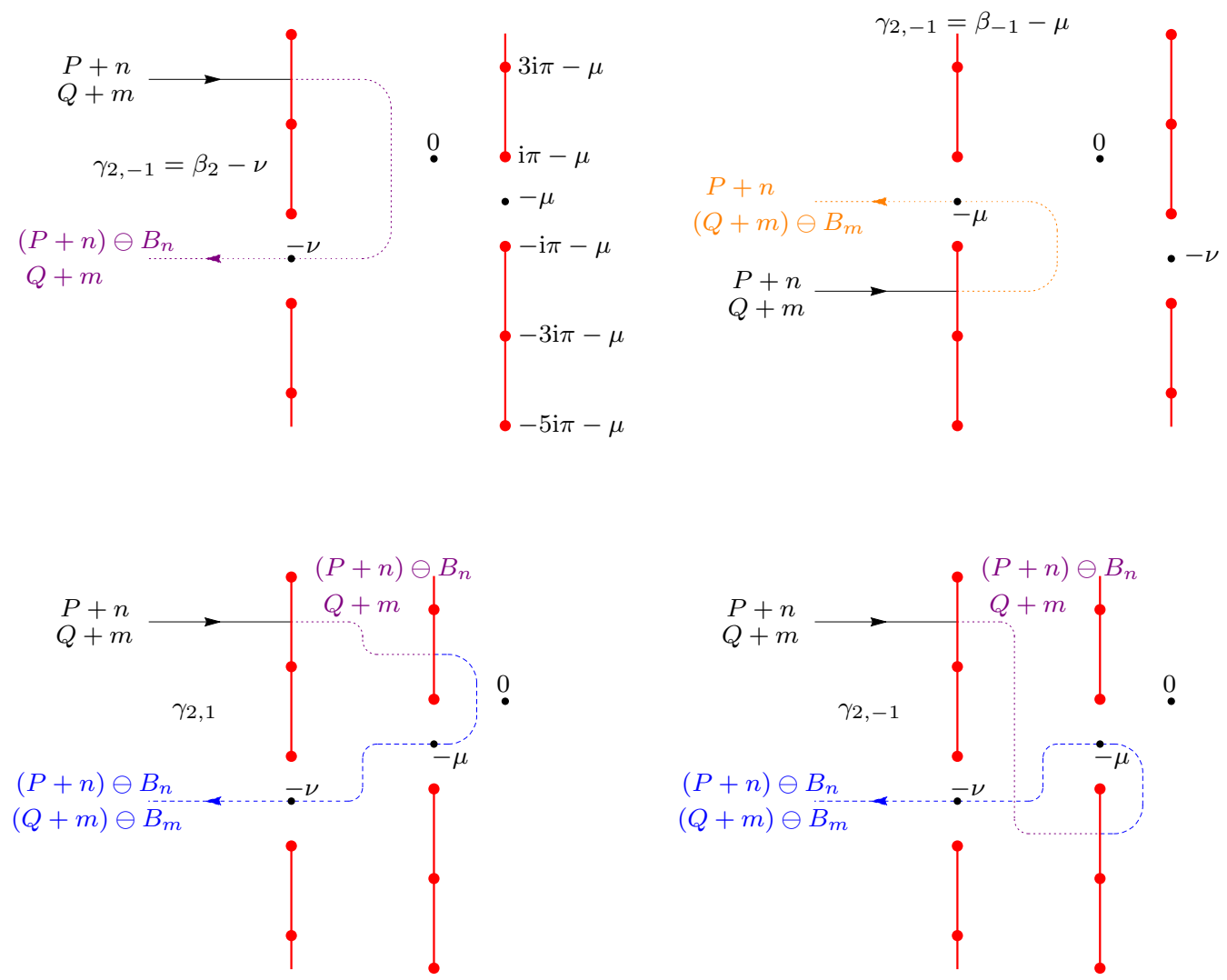

Figure 23: Path $\gamma_{n, m}$ in 102 plotted for some choices of $\nu, \mu \in \mathbb{D}, n, m \in \mathbb{Z}$. From left to right, top to bottom, the graphs represent $\gamma_{2,-1}=\beta_{2}-\nu$ for $\operatorname{Re} \mu<0<\operatorname{Re} \nu, \gamma_{2,-1}=$ $\beta_{-1}-\mu$ for $\operatorname{Re} \nu<0<\operatorname{Re} \mu, \gamma_{2,1}$ for $0<\operatorname{Re} \mu<\operatorname{Re} \nu$, and $\gamma_{2,-1}$ for $0<\operatorname{Re} \mu<\operatorname{Re} \nu$. The smaller, black dots represent the points $0,-\nu,-\mu$. The bigger, red dots represent the branch points $-\nu+2 \mathrm{i} \pi a,-\mu+2 \mathrm{i} \pi a, a \in \mathbb{Z}+1 / 2$ of $\chi_{P+n}^{\prime \prime}(\cdot+\nu) \chi_{Q+m}^{\prime \prime}(\cdot+\mu)$, and the vertical, red lines the associated branch cuts. The solid / dotted / dashed portions of the path $\gamma_{n, m}$ correspond to distinct values of the sets $\hat{P}, \hat{Q}$ in $\chi_{\hat{P}}^{\prime \prime}(u+\nu) \chi_{\hat{Q}}^{\prime \prime}(u+\mu)=$ $\mathcal{A}_{u}\left(\chi_{P+n}^{\prime \prime}(\cdot+\nu) \chi_{Q+m}^{\prime \prime}(\cdot+\mu)\right)$ which are indicated next to the paths. 
It is sufficient for the following to consider shifts of $\nu$ and $\mu$ separately, and compute the integrals over the paths $\gamma_{n, 0}$ and $\gamma_{0, m}$ only. Anticipating the result, we define the function

$$
W_{P, Q}(z)=2|P| I_{0}(z)+2 \sum_{a \in P} \sum_{b \in Q} \log \left(\frac{\kappa_{b-a+1 / 2}(z)}{\sqrt{8}}\right),
$$

and the coefficients

$$
Z_{P, Q}=\sum_{a \in P}\left(-\mathrm{i} \pi(a-1 / 2)+2 \mathrm{i} \pi \operatorname{sgn}(a)\left|Q \cap B_{a-1 / 2}\right|\right) .
$$

The function $W_{P, Q}$ is analytic in $\mathbb{D}$ so that $(\nu, \mu) \mapsto W_{P, Q}(\mu-\nu+\mathrm{i} \pi)$ and $(\nu, \mu) \mapsto$ $W_{P, Q}(\nu-\mu-\mathrm{i} \pi)$ are both analytic in $\mathbb{D}_{2}$. Additionally, using (54), (72) and $\operatorname{sgn}(n) \mid(Q-$ $a+1 / 2) \cap B_{n}|=\operatorname{sgn}(n+a)| Q \cap B_{n+a-1 / 2}|-\operatorname{sgn}(a)| Q \cap B_{a-1 / 2} \mid$, one has

$$
W_{P, Q}(z+2 \mathrm{i} \pi n)=W_{P+n, Q}(z)+1_{\{\operatorname{Re} z>0\}}\left(Z_{P+n, Q}-Z_{P, Q}\right) .
$$

The integral over $\gamma_{n, 0}$ is computed in appendix B. There, one finds

$$
\begin{aligned}
& \frac{1}{2} f_{\gamma_{n, 0}} \mathrm{~d} u \mathcal{A}_{u}\left(\chi_{P+n}^{\prime \prime}(\cdot+\nu) \chi_{Q}^{\prime \prime}(\cdot+\mu)\right) \\
& =1_{\{\operatorname{Re} \nu>0\}}\left(W_{(P+n) \ominus B_{n}, Q}(\mu-\nu+\mathrm{i} \pi)-W_{P+n, Q}(\mu-\nu+\mathrm{i} \pi)\right. \\
& \left.\quad+1_{\{\operatorname{Re} \mu>\operatorname{Re} \nu\}}\left(Z_{(P+n) \ominus B_{n}, Q}-Z_{P+n}, Q\right)\right)
\end{aligned}
$$

and

$$
\begin{aligned}
& \frac{1}{2} f_{\gamma_{0, m}} \mathrm{~d} u \mathcal{A}_{u}\left(\chi_{P}^{\prime \prime}(\cdot+\nu) \chi_{Q+m}^{\prime \prime}(\cdot+\mu)\right) \\
& =1_{\{\operatorname{Re} \mu>0\}}\left(W_{(Q+m) \ominus B_{m}+1, P}(\nu-\mu-\mathrm{i} \pi)-W_{Q+m+1, P}(\nu-\mu-\mathrm{i} \pi)\right. \\
& \left.\quad+1_{\{\operatorname{Re} \nu>\operatorname{Re} \mu\}}\left(Z_{(Q+m) \ominus B_{m}+1, P}-Z_{Q+m+1, P}\right)\right) .
\end{aligned}
$$

As usual, it is useful to interpret 102, 106, 107) in terms of translation operators mapping $K_{P, Q}$ to other functions analytic in $\mathbb{D}_{2}$. We define the operators $T_{i_{1 \mid \mathrm{r}}^{ \pm}}^{n}$ with $i=1$ corresponding to translation in the first variable, $i=2$ to translation in the second variable, and the indices $\mathrm{l} \mid \mathrm{r}, \pm$ indicating the sector for $(\operatorname{Re} \nu, \operatorname{Re} \mu)$ in which the translation is initially applied before reconstructing functions defined in $\mathbb{D}_{2}$ by analytic continuation, see table 1. All eight operators are in principle distinct, even though some of them coincide on $K_{P, Q}$. Writing $\nu$ for the first variable and $\mu$ for the second variable of the function $K_{P, Q}$, one has

$$
\begin{aligned}
T_{1_{1}^{ \pm}}^{-n} K_{P, Q}= & K_{P+n, Q} \\
T_{1_{\mathrm{r}}^{-}}^{-n} K_{P, Q}=K_{(P+n) \ominus B_{n}, Q} & +W_{(P+n) \ominus B_{n}, Q}(\mu-\nu+\mathrm{i} \pi)-W_{P+n, Q}(\mu-\nu+\mathrm{i} \pi) \\
& +Z_{(P+n) \ominus B_{n}, Q}-Z_{P+n, Q} \\
T_{1_{\mathrm{r}}^{+}}^{-n} K_{P, Q}=K_{(P+n) \ominus B_{n}, Q} & +W_{(P+n) \ominus B_{n}, Q}(\mu-\nu+\mathrm{i} \pi)-W_{P+n, Q}(\mu-\nu+\mathrm{i} \pi)
\end{aligned}
$$

for translations in the first variable and

$$
\begin{aligned}
T_{2_{1}^{ \pm}}^{-m} K_{P, Q}= & K_{P, Q+m} \\
T_{2_{\mathrm{r}}^{-}}^{-m} K_{P, Q}=K_{P,(Q+m) \ominus B_{m}} & +W_{(Q+m) \ominus B_{m}+1, P}(\nu-\mu-\mathrm{i} \pi)-W_{Q+m+1, P}(\nu-\mu-\mathrm{i} \pi) \\
& +Z_{(Q+m) \ominus B_{m}+1, P}-Z_{Q+m+1, P}
\end{aligned}
$$




\begin{tabular}{ccc}
\multicolumn{2}{c}{ Operators } & Sector \\
$T_{1_{1}^{-}}^{n}$ & $A_{n}^{1_{1}^{-}}$ & $\operatorname{Re} \nu<\min (0, \operatorname{Re} \mu)$ \\
$T_{1_{1}^{+}}^{n}$ & $A_{n}^{1_{1}^{+}}$ & $\operatorname{Re} \mu<\operatorname{Re} \nu<0$ \\
$T_{1_{\mathrm{r}}^{-}}^{n}$ & $A_{n}^{1_{\mathrm{r}}^{-}}$ & $0<\operatorname{Re} \nu<\operatorname{Re} \mu$ \\
$T_{1_{\mathrm{r}}^{+}}^{n}$ & $A_{n}^{1_{\mathrm{r}}^{+}}$ & $\max (0, \operatorname{Re} \mu)<\operatorname{Re} \nu$ \\
$T_{2_{1}^{-}}^{m}$ & $A_{m}^{2_{1}^{-}}$ & $\operatorname{Re} \mu<\min (0, \operatorname{Re} \nu)$ \\
$T_{2_{1}^{+}}^{m}$ & $A_{m}^{2_{1}^{+}}$ & $\operatorname{Re} \nu<\operatorname{Re} \mu<0$ \\
$T_{2_{\mathrm{r}}^{-}}^{m}$ & $A_{m}^{2_{\mathrm{r}}^{-}}$ & $0<\operatorname{Re} \mu<\operatorname{Re} \nu$ \\
$T_{2_{\mathrm{r}}^{+}}^{m}$ & $A_{m}^{2_{\mathrm{r}}^{+}}$ & $\max (0, \operatorname{Re} \nu)<\operatorname{Re} \mu$
\end{tabular}

Table 1: Translation operators $T^{n}, T^{m}$ and analytic continuation operators $A_{n}, A_{m}$ defined on functions $f$ analytic in $\mathbb{D}_{2}$. The translation operators verify $\left(T^{n} f\right)(\nu, \mu)=f(\nu+2 \mathrm{i} \pi n, \mu)$ (first four operators) or $\left(T^{m} f\right)(\nu, \mu)=f(\nu, \mu+2 \mathrm{i} \pi m)$ (last four operators) in the specified sector for $(\operatorname{Re} \nu, \operatorname{Re} \mu)$. The operators $A_{n}$ (first four operators) correspond to analytic continuation in the first variable across the cut $\nu \in(2 \mathrm{i} \pi(n-1 / 2), 2 \mathrm{i} \pi(n+1 / 2))$ and the operators $A_{m}$ (last four operators) correspond to analytic continuation in the second variable across the cut $\mu \in(2 \mathrm{i} \pi(m-1 / 2), 2 \mathrm{i} \pi(m+1 / 2))$, starting from the specified sector for $(\operatorname{Re} \nu, \operatorname{Re} \mu)$.

for translations in the second variable.

Analytic continuation for the first and second variable across the branch cuts ( $2 \mathrm{i} \pi(n-$ $1 / 2), 2 \mathrm{i} \pi(n+1 / 2))$ can be expressed in terms of operators $A_{n}^{i_{1 \mathrm{r}}^{ \pm}}$, with $i=1$ and $i=2$ corresponding to analytic continuation respectively in the first and in the second variable, and the indices $1 \mid \mathrm{r}, \pm$ indicating the sector for $(\operatorname{Re} \nu, \operatorname{Re} \mu)$ from which the analytic continuation is performed, see table 1. The same reasoning as in section 4.1 gives

$$
\begin{aligned}
& A_{n}^{i_{1}^{ \pm}}=T_{i_{\mathrm{r}}^{ \pm}}^{-n} T_{i_{1}^{ \pm}}^{n} \\
& A_{n}^{i_{\mathrm{r}}^{ \pm}}=T_{i_{1}^{ \pm}}^{-n} T_{i_{\mathrm{r}}^{ \pm}}^{n}
\end{aligned}
$$

for $i=1,2$. Using (108), 109 , we observe that the analytic continuation for $K_{P, Q}$ is in fact independent of the side $1 \mid \mathrm{r}$ from which the analytic continuation is made, and we simply write $A_{n}^{i^{ \pm}}$instead of $A_{n}^{i_{1 \mid \mathrm{r}}^{\mid}}$. Writing $\nu$ for the first variable and $\mu$ for the second variable of $K_{P, Q}$, we obtain

$$
\begin{aligned}
& A_{n}^{1^{-}}\left(K_{P, Q}+W_{P, Q}(\mu-\nu+\mathrm{i} \pi)+Z_{P, Q}\right)=K_{P \ominus B_{n}, Q}+W_{P \ominus B_{n}, Q}(\mu-\nu+\mathrm{i} \pi)+Z_{P \ominus B_{n}, Q} \\
& A_{n}^{1^{+}}\left(K_{P, Q}+W_{P, Q}(\mu-\nu+\mathrm{i} \pi)\right)=K_{P \ominus B_{n}, Q}+W_{P \ominus B_{n}, Q}(\mu-\nu+\mathrm{i} \pi)
\end{aligned}
$$

for analytic continuations in the first variable and

$$
\begin{aligned}
A_{n}^{2^{-}}\left(K_{P, Q}+W_{Q+1, P}(\nu-\mu-\mathrm{i} \pi)\right. & \left.+Z_{Q+1, P}\right) \\
& =K_{P, Q \ominus B_{n}}+W_{Q \ominus B_{n}+1, P}(\nu-\mu-\mathrm{i} \pi)+Z_{Q \ominus B_{n}+1, P} \\
A_{n}^{2^{+}}\left(K_{P, Q}+W_{Q+1, P}(\nu-\mu-\mathrm{i} \pi)\right) & =K_{P, Q \ominus B_{n}}+W_{Q \ominus B_{n}+1, P}(\nu-\mu-\mathrm{i} \pi)
\end{aligned}
$$

for analytic continuations in the second variable. In particular, one has $\left(A_{n}^{1^{-}}\right)^{2} K_{P, Q}=$ $\left(A_{n}^{1^{+}}\right)^{2} K_{P, Q}=\left(A_{n}^{2^{-}}\right)^{2} K_{P, Q}=\left(A_{n}^{2^{+}}\right)^{2} K_{P, Q}=K_{P, Q}$, and the branch points $\nu=2 \mathrm{i} \pi a$, $\mu=2 \mathrm{i} \pi a, a \in \mathbb{Z}+1 / 2$ of $K_{P, Q}$ are of square root type. 
Analytic continuation for $\nu$ across $(\mu+2 \mathrm{i} \pi n, \mu+2 \mathrm{i} \pi(n+1))$ is represented by the operator $D_{n}^{1, \pm}$ (respectively $D_{n}^{\mathrm{r}, \pm}$ ) if the analytic continuation is made from the sector $\operatorname{Re} \nu<0$ (resp. $\operatorname{Re} \nu>0$ ), the sign - corresponding to $\nu$ crossing the cut from the left and the sign + from the right. Analytic continuation for $\mu$ across $(\nu+2 \mathrm{i} \pi(n-1), \nu+2 \mathrm{i} \pi n)$ is represented by the operator $D_{-n}^{1, \pm}$ (respectively $D_{-n}^{\mathrm{r}, \pm}$ ) if the analytic continuation is made from the sector $\operatorname{Re} \mu<0$ (resp. $\operatorname{Re} \mu>0$ ). In terms of translation operators, one has

$$
\begin{aligned}
& D_{n}^{\mathrm{l}, \pm}=T_{1_{1}^{\mp}}^{-n} T_{1_{1}^{ \pm}}^{n} \\
& D_{n}^{\mathrm{r}, \pm}=T_{1_{\mathrm{r}}^{\mp}}^{-n} T_{1_{\mathrm{r}}^{ \pm}}^{n},
\end{aligned}
$$

and one finds

$$
\begin{aligned}
& D_{n}^{\mathrm{l}, \pm} K_{P, Q}=K_{P, Q} \\
& D_{n}^{\mathrm{r},-} K_{P, Q}=K_{P, Q}+Z_{P \ominus B_{n}-n, Q}-Z_{P-n, Q} \\
& D_{n}^{\mathrm{r},+} K_{P, Q}=K_{P, Q}-Z_{P \ominus B_{n}-n, Q}+Z_{P-n, Q} .
\end{aligned}
$$

\subsubsection{Functions $K_{P, Q}^{\Delta, \Gamma}$}

We now introduce a generalization of $K_{P, Q}$ depending on two finite sets $\Delta, \Gamma \sqsubset \mathbb{Z}+1 / 2$ similar to the generalization from $J_{P}$ to $J_{P}^{\Delta}$ in section 4.9.2. We define

$$
K_{P, Q}^{\Delta, \Gamma}(\nu, \mu)=\frac{1}{2} f_{-\infty}^{0} \mathrm{~d} u \chi_{P}^{\prime \prime \Delta}(u+\nu) \chi_{Q}^{\prime \prime \Gamma}(u+\mu),
$$

with $\chi_{P}^{\Delta}$ defined in 87 . We show in the following that $\mathrm{e}^{2 K_{P, Q}^{\Delta, \Gamma}}$ can be extended to a meromorphic function on $\mathcal{R}^{\Delta} \times \mathcal{R}^{\Gamma}$. More precisely, introducing the function

$$
\begin{aligned}
& \Upsilon_{P, Q}^{\Delta, \Gamma}(\nu, \mu)=\frac{\mathrm{e}^{2 K_{P, Q}^{\Delta, \Gamma}(\nu, \mu)}}{\left(1-\mathrm{e}^{\mu-\nu}\right)^{|P \backslash \Delta|}\left(1-\mathrm{e}^{\nu-\mu}\right)^{|Q \backslash \Gamma|}} \\
& \times \frac{\left(\prod_{a \in P \backslash \Delta} \prod_{b \in Q \backslash \Gamma}\left(\frac{2 \mathrm{i} \pi b-\mu}{4}-\frac{2 \mathrm{i} \pi a-\nu}{4}\right)\right)\left(\prod_{a \in P \cup \Delta} \prod_{b \in Q \cup \Gamma}\left(\frac{2 \mathrm{i} \pi b-\mu}{4}-\frac{2 \mathrm{i} \pi a-\nu}{4}\right)\right)}{\left(\prod_{a \in \Delta} \prod_{b \in \Gamma}\left(\frac{2 \mathrm{i} \pi b-\mu}{4}-\frac{2 \mathrm{i} \pi a-\nu}{4}\right)\right)},
\end{aligned}
$$

which verifies $\Upsilon_{P, Q}^{\Delta, \Gamma}(\nu, \mu)=\Upsilon_{P \backslash \Delta, Q \backslash \Gamma}^{\Delta, \Gamma}(\nu, \mu)$, the function $\mathrm{e}^{2 K^{\Delta, \Gamma}}$ defined by

$$
\left(\mathrm{e}^{2 K^{\Delta, \Gamma}}\right)([\nu, P],[\mu, Q])=\Upsilon_{P, Q}^{\Delta, \Gamma}(\nu, \mu)
$$

is shown below to be meromorphic on $\mathcal{R}^{\Delta} \times \mathcal{R}^{\Gamma}$ when $|\Delta| \in 2 \mathbb{N}$ and $|\Gamma| \in 2 \mathbb{N}$, which is the case needed for KPZ fluctuations.

The function $K_{P, Q}^{\Delta, \Gamma}$ can be expressed in terms of $K_{P, Q}$ as

$$
K_{P, Q}^{\Delta, \Gamma}(\nu, \mu)=\frac{K_{P, Q}(\nu, \mu)}{4}+\frac{K_{P \ominus \Delta, Q}(\nu, \mu)}{4}+\frac{K_{P, Q \ominus \Gamma}(\nu, \mu)}{4}+\frac{K_{P \ominus \Delta, Q \ominus \Gamma}(\nu, \mu)}{4},
$$


1424 1425

1426

1427

and verifies $K_{P, Q}^{\Delta, \Gamma}(\nu, \mu)=K_{P \backslash \Delta, Q \backslash \Gamma}^{\Delta, \Gamma}(\nu, \mu)$. Tedious computations using 108, 109, , 112, (113), 115) then lead for shifts by integer multiples of $2 \mathrm{i} \pi$ to

$$
\begin{array}{r}
T_{1_{1}^{ \pm}}^{-n} \Upsilon_{P, Q}^{\Delta, \Gamma}=\Upsilon_{P+n, Q}^{\Delta+n, \Gamma} \\
T_{1_{\mathrm{r}}^{ \pm}}^{-n} \Upsilon_{P, Q}^{\Delta, \Gamma}=\Upsilon_{(P+n) \ominus\left(B_{n} \backslash(\Delta+n)\right), Q}^{\Delta+n, \Gamma} \\
T_{2_{1}^{ \pm}}^{-n} \Upsilon_{P, Q}^{\Delta, \Gamma}=\Upsilon_{P, Q+n}^{\Delta, \Gamma+n} \\
T_{2_{\mathrm{r}}^{ \pm}}^{-n} \Upsilon_{P, Q}^{\Delta, \Gamma}=(-1)^{|\Delta|\left|B_{-n} \backslash \Gamma\right|} \Upsilon_{P,(Q+n) \ominus\left(B_{n} \backslash(\Gamma+n)\right)}^{\Delta, \Gamma+n}
\end{array}
$$

and for the analytic continuations to ${ }^{20}$

$$
\begin{array}{r}
A_{n}^{1} \Upsilon_{P, Q}^{\Delta, \Gamma}=\Upsilon_{P \ominus\left(B_{n} \backslash \Delta\right), Q}^{\Delta, \Gamma} \\
A_{n}^{2} \Upsilon_{P, Q}^{\Delta, \Gamma}=(-1)^{|\Delta|\left|B_{n} \backslash \Gamma\right|} \Upsilon_{P, Q \ominus\left(B_{n} \backslash \Gamma\right)}^{\Delta, \Gamma} \\
D_{n} \Upsilon_{P, Q}^{\Delta, \Gamma}=\Upsilon_{P, Q}^{\Delta, \Gamma} .
\end{array}
$$

The signs above disappear when $|\Delta|$ and $|\Gamma|$ are even numbers, and thus $\Upsilon_{P, Q}^{\Delta, \Gamma}$ can indeed be extended to a function meromorphic on $\mathcal{R}^{\Delta} \times \mathcal{R}^{\Gamma}$ in that case.

Additionally, extending $\mathrm{e}^{2 K^{\Delta, \Gamma}}$ to the collection $\overline{\mathcal{R}}^{2}$ of all $\mathcal{R}^{\Delta} \times \mathcal{R}^{\Gamma}$ by $\overline{\mathrm{e}^{2 K}}([\nu,(P, \Delta)]$, $[\mu,(Q, \Gamma)])=\mathrm{e}^{2 K^{\Delta, \Gamma}}([\nu, P],[\mu, Q])$, see section 3.8.4 the function $\overline{\mathrm{e}^{2 K}}$ verifies from 120 the symmetries $\overline{\mathrm{e}^{2 K}} \circ(\overline{\mathcal{T}} \otimes 1)=\mathrm{e}^{2 K} \circ(1 \otimes \overline{\mathcal{T}})=\mathrm{e}^{2 K}$ with $\overline{\mathcal{T}}$ defined in 44).

\section{Relation with known formulas for KPZ}

In this section, we show that several known results about KPZ fluctuations in finite volume with periodic boundary conditions are equivalent to the results given in section 2 in terms of the Riemann surfaces introduced in section 3.8 and meromorphic functions on them from section 4. For flat initial condition considered in section 2.2 , only the Riemann surface $\check{\mathcal{R}}$ is needed. For sharp wedge and stationary initial conditions, discussed in sections 2.3, 2.4 and 2.5, a summation over the Riemann surfaces $\mathcal{R}^{\Delta}, \Delta \sqsubset \mathbb{Z}+1 / 2$ is needed.

\subsection{Flat initial condition}

In this section, we show that the expression (4) for the probability of the KPZ height with flat initial condition is equivalent to exact results from [39,40].

\subsubsection{Relation with the generating function of the height}

The expression (4) follows directly from that of the generating function $\left\langle\mathrm{e}^{s h(x, t)}\right\rangle_{\text {flat }}, s>0$ obtained in [39], equation (8) ${ }^{21}$, based on earlier works [44, 45, 53, 54, 87, 90] on the Bethe ansatz solution of TASEP with periodic boundaries. One has

$$
\left\langle\mathrm{e}^{s h(x, t)}\right\rangle_{\text {flat }}=s \sum_{\substack{P \sqsubset \mathbb{Z}+1 / 2 \\|P|_{+}=|P|_{-}}} \frac{V_{P}^{2}}{4^{|P|}} \frac{\mathrm{e}^{t \chi_{P}\left(\nu_{P}(s)\right)} \mathrm{e}^{\frac{1}{2} f_{-\infty}^{\nu_{P}(s)}} \mathrm{d} v \chi_{P}^{\prime \prime}(v)^{2}}{\chi_{P}^{\prime \prime}\left(\nu_{P}(s)\right) \mathrm{e}^{\nu_{P}(s) / 4}\left(1+\mathrm{e}^{-\nu_{P}(s)}\right)^{1 / 4}},
$$

\footnotetext{
${ }^{20}$ We write $A_{n}^{i}$ instead of $A_{n}^{i_{1 \mid \mathrm{r}}^{ \pm}}$and $D_{n}$ instead of $D_{n}^{1 \mid \mathrm{r}, \pm}$ when analytic continuations depends neither on the signs of the real parts of the variables nor on the sign of the real part of their difference.

${ }^{21}$ The function $\kappa_{a}$ was called $\omega_{a}$ in 39 .
} 
where $\nu_{P}(s)$ is the solution of the equation $\chi_{P}^{\prime}\left(\nu_{P}(s)\right)=s$, conjectured to be unique and to verify $\operatorname{Re} \nu_{P}(s)>0$ when $s>0$. The probability density $p_{\text {flat }}(u)=-\partial_{u} \mathbb{P}_{\text {flat }}(h(x, t)>u)$ is related by Fourier transform to the generating function as $p_{\text {flat }}(u)=\int_{-\infty}^{\infty} \frac{\mathrm{d} s}{2 \pi} \mathrm{e}^{-\mathrm{isu}}\left\langle\mathrm{e}^{i s h(x, t)}\right\rangle_{\text {flat }}$. Making the change of variables $s=-\mathrm{i} \chi_{P}^{\prime}(\nu)$ and integrating with respect to $u$ then gives for $c>0$

$$
\mathbb{P}_{\text {flat }}(h(x, t)>u)=\sum_{\substack{P \subset \mathbb{Z}+1 / 2 \\|P|_{+}=|P|_{-}}} \frac{V_{P}^{2}}{4^{|P|}} \int_{c-\mathrm{i} \infty}^{c+\mathrm{i} \infty} \frac{\mathrm{d} \nu}{2 \mathrm{i} \pi} \frac{\mathrm{e}^{t \chi_{P}(\nu)-u \chi_{P}^{\prime}(\nu)+\frac{1}{2} f_{-\infty}^{\nu} \mathrm{d} v \chi_{P}^{\prime \prime}(v)^{2}}}{\mathrm{e}^{\nu / 4}\left(1+\mathrm{e}^{-\nu}\right)^{1 / 4}} .
$$

At this stage, there are two differences between (123) and (4): the constraint $|P|_{+}=|P|_{-}$ that $P$ has as many positive and negative elements, and the integration range $(c-\mathrm{i} \infty, c+$ $\mathrm{i} \infty)$ instead of $(c-\mathrm{i} \pi, c+\mathrm{i} \pi)$. These differences correspond simply to distinct ways to label the sheets of $\check{\mathcal{R}}$, or equivalently to the choice of a fundamental domain in $\mathcal{R}$ for the group action $\check{\mathfrak{g}}$ when writing $\check{\mathcal{R}}=\mathcal{R} / \check{\mathfrak{g}}$, compare the two representations of $\mathcal{R}$ in figure 13 on the right side $\operatorname{Re} \nu>0$ of the branch cuts. Using (69), the expressions (123) and (4) are equivalent explicit representations of (1) when $c>0$ since $|P|_{+}=|P|_{-}$implies that $|P|$ is even.

\subsubsection{Relation with Fredholm determinants}

The probability distribution $\mathbb{P}_{\text {flat }}(h(x, t)>u)$ was also expressed in [39] as the integral over $\nu, \operatorname{Re} \nu>0$ of a Fredholm determinant, using the Cauchy determinant identity (21), see section 2.6.3. Another Fredholm determinant expression for $\mathbb{P}_{\text {flat }}(h(x, t)>u)$ was proved by Baik and Liu in [40] using the propagator approach [91, 92], but with a slightly different kernel and an integral over $\nu, \operatorname{Re} \nu<0$ instead. Although it had been checked numerically that both expressions do agree, a proper derivation was missing. We show below that the expression in [40] agrees with (4) when $c<0$. The analyticity on the cylinder, consequence of the trace in (1), justifies that (4) gives the same result for $c<0$ and $c>0$, and shows that the expressions in $[39]$ and $[40]$ for flat initial condition are indeed equivalent.

We start with equation (4.2) of [40]. In our notations, Baik and Liu prove that the height function $h(x, t)$ for the totally asymmetric simple exclusion process with flat initial condition, appropriately rescaled according to KPZ universality, has the cumulative distribution function $\mathbb{P}_{\text {flat }}(h(x, t)>u)=F_{1}(-u ; t)$, with

$$
F_{1}(-u ; t)=\oint_{|z|<1} \frac{\mathrm{d} z}{2 \mathrm{i} \pi z} \mathrm{e}^{-u A_{1}(z)+t A_{2}(z)+A_{3}(z)+B(z)} \operatorname{det}\left(1-\mathcal{K}_{z}^{(1)}\right) .
$$

The contour of integration encircles 0 once in the anti-clockwise direction. Writing $z=$ $-\mathrm{e}^{\nu}, \operatorname{Re} \nu<0$, one has in terms of the functions of section 4 the identifications $A_{1}\left(-\mathrm{e}^{\nu}\right)=$ $\chi_{\emptyset}^{\prime}(\nu), A_{2}\left(-\mathrm{e}^{\nu}\right)=\chi_{\emptyset}(\nu), A_{3}\left(-\mathrm{e}^{\nu}\right)=I_{0}(\nu)$ and $B\left(-\mathrm{e}^{\nu}\right)=J_{\emptyset}(\nu)$. After some harmless changes of notations using the fact that any $\xi \in S_{z \text {, left }}$ in 40 is of the form $-\kappa_{a}(\nu)$ for some $a \in \mathbb{Z}+1 / 2$, the discrete operator $\mathcal{K}_{z}^{(1)}$ has for kernel

$$
\mathcal{K}_{z}^{(1)}(a, b)=\frac{\exp \left(\frac{2 t}{3} \kappa_{a}(\nu)^{3}+2 u \kappa_{a}(\nu)+2 \int_{-\infty}^{\nu} \mathrm{d} v \frac{\chi_{\emptyset}^{\prime \prime}(v)}{\kappa_{a}(v)}\right)}{\kappa_{a}(\nu)\left(\kappa_{a}(\nu)+\kappa_{b}(\nu)\right)},
$$

with $a, b \in \mathbb{Z}+1 / 2$ and a path of integration contained in $\mathbb{D}$. The rest of the section is essentially a more detailed version of the derivation of equation 22 , run backwards. 
1483

1484

1485

Expanding the Fredholm determinant in (124) as

$$
\operatorname{det}\left(1-\mathcal{K}_{z}^{(1)}\right)=\sum_{P \sqsubset \mathbb{Z}+1 / 2}(-1)^{|P|} \operatorname{det}\left(\mathcal{K}_{z}^{(1)}(a, b)\right)_{a, b \in P},
$$

using the Cauchy determinant identity (21) and making the change of variable $z=-\mathrm{e}^{\nu}$, one finds for any real number $c<0$

$$
\begin{aligned}
F_{1}(-u ; t) & =\int_{c-\mathrm{i} \pi}^{c+\mathrm{i} \pi} \frac{\mathrm{d} \nu}{2 \mathrm{i} \pi} \mathrm{e}^{t \chi_{\emptyset}(\nu)-u \chi_{\emptyset}^{\prime}(\nu)+I_{0}(\nu)+J_{\emptyset}(\nu)} \sum_{P \sqsubset \mathbb{Z}+1 / 2}(-1)^{|P|} \\
& \times\left(\prod_{a \in P} \frac{\exp \left(\frac{2 t}{3} \kappa_{a}(\nu)^{3}+2 u \kappa_{a}(\nu)+2 \int_{-\infty}^{\nu} \mathrm{d} v \frac{\chi_{\emptyset}^{\prime \prime}(v)}{\kappa_{a}(v)}\right)}{\kappa_{a}(\nu)}\right) \frac{\prod_{\substack{a, b \in P \\
a>b}}\left(\kappa_{a}(\nu)-\kappa_{b}(\nu)\right)^{2}}{\prod_{a, b \in P}\left(\kappa_{a}(\nu)+\kappa_{b}(\nu)\right)} .
\end{aligned}
$$

In terms of the functions $\chi_{P}$, we obtain from (64), (51)

$$
\begin{gathered}
F_{1}(-u ; t)=\int_{c-\mathrm{i} \pi}^{c+\mathrm{i} \pi} \frac{\mathrm{d} \nu}{2 \mathrm{i} \pi} \sum_{P \sqsubset \mathbb{Z}+1 / 2}(-1)^{|P|} \mathrm{e}^{t \chi_{P}(\nu)-u \chi_{P}^{\prime}(\nu)+I_{0}(\nu)+J_{\emptyset}(\nu)+\int_{-\infty}^{\nu} \mathrm{d} v \chi_{\emptyset}^{\prime \prime}(v)\left(\chi_{P}^{\prime \prime}(v)-\chi_{\emptyset}^{\prime \prime}(v)\right)} \\
\times\left(\prod_{a \in P} \frac{1}{\kappa_{a}(\nu)}\right) \frac{\prod_{\substack{a, b \in P \\
a>b}}\left(\kappa_{a}(\nu)-\kappa_{b}(\nu)\right)^{2}}{\prod_{a, b \in P}\left(\kappa_{a}(\nu)+\kappa_{b}(\nu)\right)}
\end{gathered}
$$

In terms of the regularized integral $f_{-\infty}^{\nu}=\lim _{\Lambda \rightarrow \infty}(\ldots) \log \Lambda+\int_{-\infty}^{\nu}$ subtracting appropriately logarithmic divergences used in the definition 74 for the functions $J_{P}$, one has

$$
\begin{aligned}
& J_{\emptyset}(\nu)+\int_{-\infty}^{\nu} \mathrm{d} v \chi_{\emptyset}^{\prime \prime}(v)\left(\chi_{P}^{\prime \prime}(v)-\chi_{\emptyset}^{\prime \prime}(v)\right) \\
& =J_{P}(\nu)-\frac{1}{2} f_{-\infty}^{\nu} \mathrm{d} v\left(\chi_{P}^{\prime \prime}(v)-\chi_{\emptyset}^{\prime \prime}(v)\right)^{2} \\
& =J_{P}(\nu)-2 \sum_{a, b \in P} f_{-\infty}^{\nu} \frac{\mathrm{d} v}{\kappa_{a}(v) \kappa_{b}(v)} \\
& =J_{P}(\nu)+\sum_{a, b \in P} \log \left(\frac{\left(\kappa_{a}(\nu)+\kappa_{b}(\nu)\right)^{2}}{8}\right),
\end{aligned}
$$

where the first equality comes from (74), the second from (161) and the third from (184) using the fact that $2 \log \left(\kappa_{a}(\nu)+\kappa_{b}(\nu)\right)=\log \left(\left(\kappa_{a}(\nu)+\kappa_{b}(\nu)\right)^{2}\right)$ when $\operatorname{Re} \nu<0$. We obtain

$$
\begin{aligned}
F_{1}(-u ; t)=\int_{c-\mathrm{i} \pi}^{c+\mathrm{i} \pi} \frac{\mathrm{d} \nu}{2 \mathrm{i} \pi} \sum_{P \sqsubset \mathbb{Z}+1 / 2} \frac{(-1)^{|P|}}{4^{|P|}} & \mathrm{e}^{t \chi_{P}(\nu)-u \chi_{P}^{\prime}(\nu)+I_{0}(\nu)+J_{P}(\nu)} \\
& \times \prod_{\substack{a, b \in P \\
a>b}}\left(\frac{\kappa_{a}(\nu)^{2}-\kappa_{b}(\nu)^{2}}{8}\right)^{2} .
\end{aligned}
$$

From the definition 50 of $\kappa_{a}(\nu)$, one has $\left(\kappa_{a}(\nu)^{2}-\kappa_{b}(\nu)^{2}\right) / 8=2 \mathrm{i} \pi a / 4-2 \mathrm{i} \pi b / 4$, which finally gives (4) with $c<0$.

\subsection{Sharp wedge initial condition}

In this section, we show that the expression 10 for the probability of the KPZ height with sharp wedge initial condition is equivalent to exact results from [39,40]. 


\subsubsection{Relation with the generating function of the height from [39]}

The expression (6) for sharp wedge initial condition is a consequence of the generating function obtained in [39], equation (9), and given for $s>0$ by

$$
\begin{aligned}
\left\langle\mathrm{e}^{s h(x, t)}\right\rangle_{\mathrm{sw}}=s \sum_{\substack{P, H \subset \mathbb{Z}+1 / 2 \\
|P|_{ \pm}=|H|_{\mp}}} \frac{\mathrm{i}^{|P|+|H|} V_{P}^{2} V_{H}^{2}}{4^{|P|+|H|}} \mathrm{e}^{2 \mathrm{i} \pi x\left(\sum_{a \in P} a-\sum_{a \in H} a\right)} & \\
\times & \frac{\mathrm{e}^{t \chi_{P, H}\left(\nu_{P, H}(s)\right)} \lim _{\Lambda \rightarrow \infty} \Lambda^{-|P|^{2}-|H|^{2}} \mathrm{e}^{\int_{-\Lambda}^{\nu_{P, H}(s)} \mathrm{d} v \chi_{P, H}^{\prime \prime}(v)^{2}}}{\chi_{P, H}^{\prime \prime}\left(\nu_{P, H}(s)\right)},
\end{aligned}
$$

with Vandermonde determinants $V_{P}, V_{H}$ defined in (5),

$$
\chi_{P, H}(v)=\frac{\chi_{P}(v)+\chi_{H}(v)}{2}
$$

and $\nu_{P, H}(s)$ the solution of $\chi_{P, H}^{\prime}\left(\nu_{P, H}(s)\right)=s$, conjectured to be unique when $\operatorname{Re} s>0$. The restrictions $|P|_{+}=|H|_{-},|P|_{-}=|H|_{+}$on the number of positive and negative elements of $P$ and $H$ imply in particular that we are summing only over sets $P$ and $H$ with $|P|=|H|$.

As in section 2.2, the cumulative distribution function of the height can be derived from the generating function by Fourier transform, and we obtain in terms of $\chi_{P}^{\Delta}, J_{P}^{\Delta}$ defined in (87), (74)

$$
\begin{aligned}
\mathbb{P}_{\mathrm{sw}}(h(x, t)>u)= & \sum_{\substack{P, H \subset \mathbb{Z}+1 / 2 \\
|P|_{ \pm}=|H|_{\mp}}} \frac{\mathrm{i}^{|P|+|H|} V_{P}^{2} V_{H}^{2}}{4^{|P|+|H|}} \mathrm{e}^{2 \mathrm{i} \pi x\left(\sum_{a \in P} a-\sum_{a \in H} a\right)} \\
& \times \int_{c-\mathrm{i} \infty}^{c+\mathrm{i} \infty} \frac{\mathrm{d} \nu}{2 \mathrm{i} \pi} \mathrm{e}^{t \chi_{P}^{\Delta}(\nu)-u \chi_{P}^{\prime \Delta}(\nu)+2 J_{P}^{\Delta}(\nu)},
\end{aligned}
$$

with $c>0$ and $\Delta=P \ominus H$.

One has $|P|+|H|=2|P \backslash \Delta|+|\Delta|, \sum_{a \in P} a-\sum_{a \in H} a=\sum_{a \in A} a-\sum_{a \in \Delta \backslash A} a$ and

$$
V_{P}^{2} V_{H}^{2}=V_{A}^{2} V_{\Delta \backslash A}^{2} \prod_{a \in P \backslash \Delta} \prod_{\substack{b \in P \cup \Delta \\ b \neq a}}\left(\frac{2 \mathrm{i} \pi a}{4}-\frac{2 \mathrm{i} \pi b}{4}\right)^{2},
$$

where $\Delta=P \ominus H$ and $A=P \cap \Delta$. These identities allow to rewrite 133) as

$$
\begin{aligned}
& \mathbb{P}_{\mathrm{sW}}(h(x, t)>u)=\sum_{\Delta \sqsubset \mathbb{Z}+1 / 2} \sum_{A \subset \Delta} \sum_{\substack{Q \subset \mathbb{Z}+1 / 2 \\
Q \cap \Delta=\emptyset}} 1_{\left\{|P|_{ \pm}=|P \ominus \Delta|_{\mp}\right\}}(\mathrm{i} / 4)^{2|P \backslash \Delta|+|\Delta|} V_{A}^{2} V_{\Delta \backslash A}^{2} \\
& \times\left(\prod_{a \in P \backslash \Delta} \prod_{\substack{b \in P \cup \Delta \\
b \neq a}}\left(\frac{2 \mathrm{i} \pi a}{4}-\frac{2 \mathrm{i} \pi b}{4}\right)^{2}\right) \mathrm{e}^{2 \mathrm{i} \pi x\left(\sum_{a \in A} a-\sum_{a \in \Delta \backslash A} a\right)} \\
& \times \int_{c-\mathrm{i} \infty}^{c+\mathrm{i} \infty} \frac{\mathrm{d} \nu}{2 \mathrm{i} \pi} \mathrm{e}^{t \chi_{P}^{\Delta}(\nu)-u \chi_{P}^{\prime \Delta}(\nu)+2 J_{P}^{\Delta}(\nu)}
\end{aligned}
$$

where the sum over $P=Q \cup A$ and $H=P \cup(\Delta \backslash A)$ has been replaced by a sum over $\Delta, A, Q$. Since $A \subset \Delta$ and the function $\chi_{P}^{\Delta}$ verifies $\chi_{P}^{\Delta}=\chi_{P \backslash \Delta}^{\Delta}$, all $\chi_{P}^{\Delta}$ in the integral can be replaced by $\chi_{Q}^{\Delta}$. Additionally, $P \backslash \Delta=Q$ and $P \cup \Delta=Q \cup \Delta$ shows that further factors are independent of $A$. Finally $|P|_{ \pm}=|Q|_{ \pm}+|A|_{ \pm},|P \ominus \Delta|_{\mp}=|Q|_{\mp}+|\Delta \backslash A|_{\mp}=$ 
1515

1516

1517

1518

1519

1520

1521

1522

1523

1524

1525

$|Q|_{\mp}+|\Delta|_{\mp}-|A|_{\mp}$, and the constraints $|P|_{ \pm}=|P \ominus \Delta|_{\mp}$ can be replaced by $|A|=|\Delta \backslash A|$, implying that $|\Delta|$ is necessarily even, and $|Q|_{+}-|Q \ominus \Delta|_{-}=-|\Delta| / 2$, equivalent to $\lambda_{+}(Q, \Delta)=-|\Delta| / 2$ with $\lambda_{+}$defined above (43).

In terms of $\Xi_{x}^{\Delta}$ defined in (7) and of the functions $\chi^{\Delta}, \chi^{\prime \Delta}, \mathrm{e}^{2 J^{\Delta}}$ on the Riemann surface $\mathcal{R}^{\Delta}$ defined in (86), 91, ,96), we obtain

$$
\begin{aligned}
\mathbb{P}_{\mathrm{sw}}(h(x, t)>u)= & \sum_{\Delta \sqsubset \mathbb{Z}+1 / 2} \Xi_{x}^{\Delta} \sum_{\substack{Q \sqsubset \mathbb{Z}+1 / 2 \\
Q \cap \Delta=\emptyset}} 1_{\left\{\lambda_{+}(Q, \Delta)=-|\Delta| / 2\right\}} \\
& \times \int_{c-\mathrm{i} \infty}^{c+\mathrm{i} \infty} \frac{\mathrm{d} \nu}{2 \mathrm{i} \pi}\left(\mathrm{e}^{t \chi^{\Delta}-u \chi^{\prime \Delta}+2 J^{\Delta}}\right)([\nu, Q]) .
\end{aligned}
$$

We split $\int_{c-\mathrm{i} \infty}^{c+\mathrm{i} \infty}$ into $\sum_{m=-\infty}^{\infty} \int_{c-2 \mathrm{i} \pi(m-1 / 2)}^{c+2 \mathrm{i}(m+1 / 2)}$ and shift $\nu$ by $-2 \mathrm{i} \pi m$. We then use the symmetry by $\overline{\mathcal{T}}$ defined in 44 of the extension to $\overline{\mathcal{R}}$ of $\chi^{\Delta}$ and $\mathrm{e}^{2 J^{\Delta}}$, i.e. we replace $[\nu-2 \mathrm{i} \pi m,(Q, \Delta)]$ by $\left[\nu,\left((Q+m) \ominus\left(B_{m} \backslash(\Delta+m)\right), \Delta+m\right)\right]$ everywhere since $c>0$. Making the change of variable $Q \rightarrow P \ominus\left(B_{m} \backslash(\Delta+m)\right)-m=(P-m) \ominus\left(B_{-m} \backslash \Delta\right)$ followed by $\Delta \rightarrow \Delta-m$, the constraint $Q \cap \Delta=\emptyset$ becomes $P \cap \Delta=\emptyset$. Using $\Xi_{x}^{\Delta-m}=\Xi_{x}^{\Delta}$ then leads to

$$
\begin{array}{r}
\mathbb{P}_{\mathrm{sW}}(h(x, t)>u)=\sum_{\Delta \sqsubset \mathbb{Z}+1 / 2} \Xi_{x}^{\Delta} \sum_{\substack{P \sqsubset \mathbb{Z}+1 / 2 \\
P \cap \Delta=\emptyset}} \sum_{m=-\infty}^{\infty} 1_{\left\{\lambda_{+}((P-m) \ominus(B-m \backslash(\Delta-m)), \Delta-m)=-|\Delta| / 2\right\}}(137) \\
\times \int_{c-\mathrm{i} \pi}^{c+\mathrm{i} \pi} \frac{\mathrm{d} \nu}{2 \mathrm{i} \pi}\left(\mathrm{e}^{t \chi^{\Delta}-u \chi^{\prime \Delta}+2 J^{\Delta}}\right)([\nu, P]) .
\end{array}
$$

Using (43), the condition $\lambda_{+}\left((P-m) \ominus\left(B_{-m} \backslash(\Delta-m)\right), \Delta-m\right)=-|\Delta| / 2$ is equivalent to $\lambda_{+}(P, \Delta)=m-|\Delta| / 2$. We observe that there exists a unique $m \in \mathbb{Z}$ such that the constraint is verified when $|\Delta|$ is even, and that no $m \in \mathbb{Z}$ satisfies the constraint when $|\Delta|$ is odd. Since $\Xi_{x}^{\Delta}=0$ when $|\Delta|$ is odd, this leads to $10 \mid$, using the definitions 86 , (96) of $\chi^{\Delta}$ and $\mathrm{e}^{2 J^{\Delta}}$.

The slightly tedious derivation of 10 from $(135)$ in this section can be understood more directly, but at the price of heavier formalism, by considering a collection $\overline{\overline{\mathcal{R}}}$ of copies of $\mathcal{R}$ and a covering map from $\overline{\overline{\mathcal{R}}}$ to $\overline{\mathcal{R}}$ projecting each copy of $\mathcal{R}$ in $\overline{\overline{\mathcal{R}}}$ to a distinct $\mathcal{R}^{\Delta}$ in $\overline{\mathcal{R}}$. The functions appearing in 135 can then be interpreted as functions on the components $\mathcal{R}$ of $\overline{\mathcal{R}}$, equal at $[\nu, P] \in \mathcal{R}$ to the product of a constant depending only on $A=P \cap \Delta$, which is eventually gathered into $\Xi_{x}^{\Delta}$, and a function of $[\nu, P \backslash \Delta] \in \mathcal{R}^{\Delta}$.

\subsubsection{Relation with the expression from Baik and Liu $\mid 40$}

In this section, we show that our result (6) for the cumulative distribution function of KPZ fluctuations with sharp wedge initial condition agrees with the alternative formula by Baik and Liu [40], with an integration on the left side of the branch cuts.

We start with equation (4.10) of [40]. In our notations, Baik and Liu prove that the height function $h(x, t)$ for the totally asymmetric simple exclusion process with domain wall initial condition, appropriately rescaled according to KPZ universality, has the cumulative distribution function $\mathbb{P}_{\mathrm{sw}}(h(x, t)>u)=F_{2}(-u ; t, x)$, with

$$
F_{2}(-u ; t, x)=\oint_{|z|<1} \frac{\mathrm{d} z}{2 \mathrm{i} \pi z} \mathrm{e}^{-u A_{1}(z)+t A_{2}(z)+2 B(z)} \operatorname{det}\left(1-\mathcal{K}_{z}^{(2)}\right) .
$$

The contour of integration encircles 0 once in the anti-clockwise direction. Writing $z=$ $-\mathrm{e}^{\nu}, \operatorname{Re} \nu<0$, one has in terms of the functions of section 4 the identifications $A_{1}\left(-\mathrm{e}^{\nu}\right)=$ 
1548

1549

1550

1551 1552

1553

1554

$\chi_{\emptyset}^{\prime}(\nu), A_{2}\left(-\mathrm{e}^{\nu}\right)=\chi_{\emptyset}(\nu)$ and $B\left(-\mathrm{e}^{\nu}\right)=J_{\emptyset}(\nu)$. After some harmless changes of notations using the fact that any $\xi \in S_{z \text {, left }}$ in $[40]$ is of the form $-\kappa_{a}(\nu)$ for some $a \in \mathbb{Z}+1 / 2$, the discrete operator $\mathcal{K}_{z}^{(2)}$ has for kernel

$$
\begin{aligned}
\mathcal{K}_{z}^{(2)}(a, b)= & \frac{\exp \left(\frac{t}{3} \kappa_{a}(\nu)^{3}+u \kappa_{a}(\nu)+2 \mathrm{i} \pi a x+2 \int_{-\infty}^{\nu} \mathrm{d} v \frac{\chi_{\emptyset}^{\prime \prime}(v)}{\kappa_{a}(v)}\right)}{\kappa_{a}(\nu)} \\
& \times \sum_{c \in \mathbb{Z}+1 / 2} \frac{\exp \left(\frac{t}{3} \kappa_{c}(\nu)^{3}+u \kappa_{c}(\nu)-2 \mathrm{i} \pi c x+2 \int_{-\infty}^{\nu} \mathrm{d} v \frac{\chi_{\emptyset}^{\prime \prime}(v)}{\kappa_{c}(v)}\right)}{\kappa_{c}(\nu)\left(\kappa_{a}(\nu)+\kappa_{c}(\nu)\right)\left(\kappa_{b}(\nu)+\kappa_{c}(\nu)\right)},
\end{aligned}
$$

with $a, b \in \mathbb{Z}+1 / 2$. The rest of the section is essentially a more detailed version of the derivation of (25), run backwards. Expanding the Fredholm determinant in (124) as

$$
\operatorname{det}\left(1-\mathcal{K}_{z}^{(2)}\right)=\sum_{P \sqsubset \mathbb{Z}+1 / 2}(-1)^{|P|} \operatorname{det}\left(\mathcal{K}_{z}^{(2)}(a, b)\right)_{a, b \in P},
$$

using

$$
\operatorname{det}\left(\sum_{c \in \mathbb{Z}+1 / 2} \mathcal{K}_{a, b, c}\right)_{a, b \in P}=\left(\prod_{a \in P} \sum_{c_{a} \in \mathbb{Z}+1 / 2}\right) \operatorname{det}\left(\mathcal{K}_{a, b, c_{a}}\right)_{a, b \in P},
$$

the Cauchy determinant identity

$$
\operatorname{det}\left(\frac{1}{\kappa_{c_{a}}+\kappa_{b}}\right)_{a, b \in P}=\frac{\left(\prod_{\substack{a, b \in P \\ a>b}}\left(\kappa_{a}-\kappa_{b}\right)\right)\left(\prod_{\substack{a, b \in P \\ a>b}}\left(\kappa_{c_{a}}-\kappa_{c_{b}}\right)\right)}{\prod_{a, b \in P}\left(\kappa_{c_{a}}+\kappa_{b}\right)},
$$

and making the change of variable $z=-\mathrm{e}^{\nu}$, one finds for any real number $c<0$

$$
\begin{aligned}
F_{2}(-u ; t, x)=\int_{c-\mathrm{i} \pi}^{c+\mathrm{i} \pi} & \frac{\mathrm{d} \nu}{2 \mathrm{i} \pi} \mathrm{e}^{t \chi_{\emptyset}(\nu)-u \chi_{\emptyset}^{\prime}(\nu)+2 J_{\emptyset}(\nu)} \sum_{P \sqsubset \mathbb{Z}+1 / 2}\left(\prod_{a \in P} \sum_{c_{a} \in \mathbb{Z}+1 / 2}\right)(-1)^{|P|} \\
& \times\left(\prod_{a \in P} \frac{\exp \left(\frac{t}{3} \kappa_{a}(\nu)^{3}+u \kappa_{a}(\nu)+2 \mathrm{i} \pi a x+2 \int_{-\infty}^{\nu} \mathrm{d} v \frac{\chi_{\emptyset}^{\prime \prime}(v)}{\kappa_{a}(v)}\right)}{\kappa_{a}(\nu)}\right) \\
& \times\left(\prod_{a \in P} \frac{\exp \left(\frac{t}{3} \kappa_{c_{a}}(\nu)^{3}+u \kappa_{c_{a}}(\nu)-2 \mathrm{i} \pi c_{a} x+2 \int_{-\infty}^{\nu} \mathrm{d} v \frac{\chi_{\emptyset}^{\prime \prime}(v)}{\kappa_{c_{a}}(v)}\right)}{\kappa_{c_{a}}(\nu)}\right) \\
& \times \frac{\left(\prod_{a, b \in P}\left(\kappa_{a>b}(\nu)-\kappa_{b}(\nu)\right)\right)\left(\prod_{\substack{a, b \in P \\
a>b}}\left(\kappa_{c_{a}}(\nu)-\kappa_{c_{b}}(\nu)\right)\right)}{\left(\prod_{a \in P}\left(\kappa_{a}(\nu)+\kappa_{c_{a}}(\nu)\right)\right)\left(\prod_{a, b \in P}\left(\kappa_{c_{a}}(\nu)+\kappa_{b}(\nu)\right)\right)} .
\end{aligned}
$$

Because of the factor $\prod_{\substack{a, b \in P \\ a>b}}\left(\kappa_{c_{a}}(\nu)-\kappa_{c_{b}}(\nu)\right)$, only the tuples $c_{a}, a \in P$ with distinct elements contribute, and one can replace these tuples by finite sets $H \sqsubset \mathbb{Z}+1 / 2$ with $|H|=|P|$ up to permutations. Using the identity

$$
\begin{aligned}
& \left(\prod_{a \in P} \sum_{c_{a} \in \mathbb{Z}+1 / 2}\right) 1_{\left\{\left\{c_{a}, a \in P\right\}=H\right\}} \frac{\left(\prod_{\substack{a, b \in P \\
a>b}}\left(\kappa_{c_{a}}-\kappa_{c_{b}}\right)\right)}{\left(\prod_{a \in P}\left(\kappa_{a}+\kappa_{c_{a}}\right)\right)\left(\prod_{a, b \in P}\left(\kappa_{c_{a}}+\kappa_{b}\right)\right)} \\
& =\frac{\left(\prod_{\substack{a, b \in P \\
a>b}}\left(\kappa_{a}-\kappa_{b}\right)\right)\left(\prod_{\substack{a, b \in H \\
a>b}}\left(\kappa_{a}-\kappa_{b}\right)^{2}\right)}{\prod_{a \in P} \prod_{b \in H}\left(\kappa_{a}+\kappa_{b}\right)^{2}}
\end{aligned}
$$


1559

for $P, H \sqsubset \mathbb{Z}+1 / 2,|P|=|H|$, (132), (64) and (161), this leads to

$$
\begin{aligned}
F_{2}(-u ; t, x)=\int_{c-\mathrm{i} \pi}^{c+\mathrm{i} \pi} \frac{\mathrm{d} \nu}{2 \mathrm{i} \pi} \sum_{\substack{P, H \subset \mathbb{Z}+1 / 2 \\
|P|=|H|}}(-1)^{|P|} \mathrm{e}^{t \chi_{P, H}(\nu)-u \chi_{P, H}^{\prime}(\nu)} \mathrm{e}^{2 \mathrm{i} \pi x\left(\sum_{a \in P} a-\sum_{a \in H} a\right)} \\
\times \mathrm{e}^{2 J_{\emptyset}(\nu)+2 \int_{-\infty}^{\nu} \mathrm{d} v \chi_{\emptyset}^{\prime \prime}(v)\left(\chi_{P, H}^{\prime \prime}(v)-\chi_{\emptyset}^{\prime \prime}(v)\right)}\left(\prod_{a \in P} \frac{1}{\kappa_{a}(\nu)}\right)\left(\prod_{a \in H} \frac{1}{\kappa_{a}(\nu)}\right) \\
\times \frac{\left(\prod_{\substack{a, b \in P \\
a>b}}\left(\kappa_{a}(\nu)-\kappa_{b}(\nu)\right)\right)^{2}\left(\prod_{\substack{a, b \in H \\
a>b}}\left(\kappa_{a}(\nu)-\kappa_{b}(\nu)\right)\right)^{2}}{\left(\prod_{a \in P} \prod_{b \in H}\left(\kappa_{a}(\nu)+\kappa_{b}(\nu)\right)\right)^{2}}
\end{aligned}
$$

In terms of the regularized integral $f_{-\infty}^{\nu}=\lim _{\Lambda \rightarrow \infty}(\ldots) \log \Lambda+\int_{-\infty}^{\nu}$ subtracting appropriately logarithmic divergences used in the definition (74) for the functions $J_{P}$, one has

$$
\begin{aligned}
& J_{\emptyset}(\nu)+\int_{-\infty}^{\nu} \mathrm{d} v \chi_{\emptyset}^{\prime \prime}(v)\left(\chi_{P, H}^{\prime \prime}(v)-\chi_{\emptyset}^{\prime \prime}(v)\right) \\
& =\frac{1}{2} f_{-\infty}^{\nu} \mathrm{d} v \chi_{P, H}^{\prime \prime}(v)^{2}-\frac{1}{2} f_{-\infty}^{\nu} \mathrm{d} v\left(\chi_{P, H}^{\prime \prime}(v)-\chi_{\emptyset}^{\prime \prime}(v)\right)^{2} \\
& =\frac{1}{2} \int_{-\infty}^{\nu} \mathrm{d} v \chi_{P, H}^{\prime \prime}(v)^{2}-\frac{1}{2} \sum_{a, b \in P} f_{-\infty}^{\nu} \frac{\mathrm{d} v}{\kappa_{a}(v) \kappa_{b}(v)}-\frac{1}{2} \sum_{a, b \in H} f_{-\infty}^{\nu} \frac{\mathrm{d} v}{\kappa_{a}(v) \kappa_{b}(v)} \\
& \quad-\sum_{a \in P} \sum_{b \in H} f_{-\infty}^{\nu} \frac{\mathrm{d} v}{\kappa_{a}(v) \kappa_{b}(v)} \\
& =\frac{1}{2} \int_{-\infty}^{\nu} \mathrm{d} v \chi_{P, H}^{\prime \prime}(v)^{2}+\frac{1}{2} \sum_{a, b \in P} \log \left(\frac{\kappa_{a}(\nu)+\kappa_{b}(\nu)}{\sqrt{8}}\right) \\
& \quad+\frac{1}{2} \sum_{a, b \in H} \log \left(\frac{\kappa_{a}(\nu)+\kappa_{b}(\nu)}{\sqrt{8}}\right)+\sum_{a \in P} \sum_{b \in H} \log \left(\frac{\kappa_{a}(\nu)+\kappa_{b}(\nu)}{\sqrt{8}}\right)
\end{aligned}
$$

where the first equality comes from (74), the second from (132), (161) and the third from (184). We obtain

$$
\begin{gathered}
F_{2}(-u ; t, x)=\int_{c-\mathrm{i} \pi}^{c+\mathrm{i} \pi} \frac{\mathrm{d} \nu}{2 \mathrm{i} \pi} \sum_{\substack{P, H \subset \mathbb{Z}+1 / 2 \\
|P|=|H|}} \frac{\mathrm{i}^{|P|+|H|}}{4^{|P|+|H|}} \mathrm{e}^{t \chi_{P, H}(\nu)-u \chi_{P, H}^{\prime}(\nu)+f_{-\infty}^{\nu} \mathrm{d} v \chi_{P, H}^{\prime \prime}(v)^{2}} \\
\times \mathrm{e}^{2 \mathrm{i} \pi x\left(\sum_{a \in P} a-\sum_{a \in H} a\right)}\left(\prod_{\substack{a, b \in P \\
a>b}} \frac{\kappa_{a}(\nu)^{2}-\kappa_{b}(\nu)^{2}}{8}\right)^{2}\left(\prod_{\substack{a, b \in H \\
a>b}} \frac{\kappa_{a}(\nu)^{2}-\kappa_{b}(\nu)^{2}}{8}\right)^{2} .
\end{gathered}
$$

From the definition 50 of $\kappa_{a}(\nu)$, one has $\left(\kappa_{a}(\nu)^{2}-\kappa_{b}(\nu)^{2}\right) / 8=2 \mathrm{i} \pi a / 4-2 \mathrm{i} \pi b / 4$, which gives

$$
\begin{array}{r}
F_{2}(-u ; t, x)=\int_{c-\mathrm{i} \pi}^{c+\mathrm{i} \pi} \frac{\mathrm{d} \nu}{2 \mathrm{i} \pi} \sum_{\substack{P, H \subset \mathbb{Z}+1 / 2 \\
|P|=|H|}} \frac{\mathrm{i}^{|P|+|H|} V_{P}^{2} V_{H}^{2}}{4^{|P|+|H|}} \mathrm{e}^{2 \mathrm{i} \pi x\left(\sum_{a \in P} a-\sum_{a \in H} a\right)} \\
\times \mathrm{e}^{t \chi_{P, H}(\nu)-u \chi_{P, H}^{\prime}(\nu)+f_{-\infty}^{\nu} \mathrm{d} v \chi_{P, H}^{\prime \prime}(v)^{2}} .
\end{array}
$$

with $V_{P}$ the Vandermonde determinant defined in (5). Introducing $\Delta=P \ominus H$ and the 
1567

functions $\chi_{P}^{\Delta}$ and $J_{P}^{\Delta}$ from (87), 92, we finally obtain

$$
\begin{array}{r}
\mathbb{P}_{\mathrm{sw}}(h(x, t)>u)=\sum_{\substack{P, H \subset \mathbb{Z}+1 / 2 \\
|P|=|H|}} \frac{\mathrm{i}^{|P|+|H|} V_{P}^{2} V_{H}^{2}}{4^{|P|+|H|}} \mathrm{e}^{2 \mathrm{i} \pi x\left(\sum_{a \in P} a-\sum_{a \in H} a\right)} \\
\times \int_{c-\mathrm{i} \pi}^{c+\mathrm{i} \pi} \frac{\mathrm{d} \nu}{2 \mathrm{i} \pi} \mathrm{e}^{t \chi_{P}^{\Delta}(\nu)-u \chi_{P}^{\prime \Delta}(\nu)+2 J_{P}^{\Delta}(\nu)},
\end{array}
$$

with $\Delta=P \ominus H$ and $V_{P}, \chi_{P}^{\Delta}, J_{P}^{\Delta}$ defined in (5), 87), (92). The sign of $c$, the integration range and the constraint on the sets $P$ and $H$ differ from (133). This corresponds simply to another choice of fundamental domain for the Riemann surfaces $\mathcal{R}^{\Delta}$. Indeed, writing $P=Q \cup A$ with $A \subset \Delta, Q \cap \Delta=\emptyset$ and using (134) as in the previous section leads to

$$
\begin{aligned}
\mathbb{P}_{\mathrm{sw}}(h(x, t)>u)= & \sum_{\Delta \sqsubset \mathbb{Z}+1 / 2} \sum_{A \subset \Delta} \sum_{\substack{Q \subset \mathbb{Z}+1 / 2 \\
Q \cap \Delta=\emptyset}} 1_{\{|P|=|P \ominus \Delta|\}}(\mathrm{i} / 4)^{2|P \backslash \Delta|+|\Delta|} V_{A}^{2} V_{\Delta \backslash A}^{2} \\
& \times\left(\prod_{a \in P \backslash \Delta} \prod_{\substack{b \in P \cup \Delta \\
b \neq a}}\left(\frac{2 \mathrm{i} \pi a}{4}-\frac{2 \mathrm{i} \pi b}{4}\right)^{2}\right) \mathrm{e}^{2 \mathrm{i} \pi x\left(\sum_{a \in A} a-\sum_{a \in \Delta \backslash A} a\right)} \\
& \times \int_{c-\mathrm{i} \pi}^{c+\mathrm{i} \pi} \frac{\mathrm{d} \nu}{2 \mathrm{i} \pi} \mathrm{e}^{t \chi_{P}^{\Delta}(\nu)-u \chi_{P}^{\prime \Delta}(\nu)+2 J_{P}^{\Delta}(\nu)}
\end{aligned}
$$

which parallels (135). Since $|P|=|P \ominus \Delta|$ is equivalent to $|A|=|\Delta \backslash A|$, the same reasoning as from 135 to (136) finally gives

$$
\mathbb{P}_{\mathrm{sW}}(h(x, t)>u)=\sum_{\Delta \sqsubset \mathbb{Z}+1 / 2} \Xi_{x}^{\Delta} \sum_{\substack{Q\llcorner\mathbb{Z}+1 / 2 \\ Q \cap \Delta=\emptyset}} \int_{c-\mathrm{i} \pi}^{c+\mathrm{i} \pi} \frac{\mathrm{d} \nu}{2 \mathrm{i} \pi}\left(\mathrm{e}^{t \chi^{\Delta}-u \chi^{\prime \Delta}+2 J^{\Delta}}\right)([\nu, Q]),
$$

which is precisely 10 .

\subsection{Multiple-time statistics with sharp wedge initial condition}

In this section, we derive (13) starting with a result by Baik and Liu [42]. We also discuss the pole structure on the Riemann surfaces $\mathcal{R}^{\Delta_{\ell}}$ of the final expression.

\subsubsection{Derivation of (13) from Baik-Liu 42}

The joint distribution of the height at multiple times $0<t_{1}<\ldots<t_{m}$ and positions $x_{j}$ was obtained by Baik and Liu in [42], equation (2.15), with the expression (2.21) for $C(\mathbf{z})$, and (2.51), (2.55) for $D(\mathbf{z})$. Under the replacements $\tau_{j} \rightarrow t_{j}, \gamma_{j} \rightarrow x_{j}, x_{j} \rightarrow-u_{j}$, 
1583

1584

one has $\mathbb{P}_{\mathrm{sw}}\left(h\left(x_{1}, t_{1}\right)>u_{1}, \ldots, h\left(x_{m}, t_{m}\right)>u_{m}\right)=F(\vec{t}, \vec{x}, \vec{u})$ where

$$
\begin{aligned}
F(\vec{t}, \vec{x}, \vec{u}) & \\
=\oint \frac{\mathrm{d} z_{1}}{2 \mathrm{i} \pi z_{1}} & \cdots \\
\times & \frac{\mathrm{d} z_{m}}{2 \mathrm{i} \pi z_{m}}\left(\prod_{n_{1}, \ldots, n_{m}=0}^{m}\left(\frac{z_{\ell}}{z_{\ell}-z_{\ell+1}} \frac{\mathrm{e}^{-u_{\ell} A_{1}\left(z_{\ell}\right)+t_{\ell} A_{2}\left(z_{\ell}\right)}}{\mathrm{e}^{-u_{\ell} A_{1}\left(z_{\ell+1}\right)+t_{\ell} A_{2}\left(z_{\ell+1}\right)}} \mathrm{e}^{2 B\left(z_{\ell}, z_{\ell}\right)-2 B\left(z_{\ell+1}, z_{\ell}\right)}\right)\right) \\
& \times\left(\prod_{\ell=1}^{m} \frac{1}{\left(n_{\ell} !\right)^{2}}\right)\left(\prod_{\ell=2}^{m}\left(\left(1-\frac{z_{\ell-1}}{z_{\ell}}\right)^{n_{\ell}}\left(1-\frac{z_{\ell}}{z_{\ell-1}}\right)^{n_{\ell-1}}\right)\right)\left(\prod_{\ell=1}^{m}\left(\frac{\Delta\left(U^{(\ell)}\right)^{2} \Delta\left(V^{(\ell)}\right)^{2}}{\Delta\left(U^{(\ell)} ; V^{(\ell)}\right)^{2}} \hat{f}_{\ell}\left(U^{(\ell)}\right) \hat{f}_{\ell}\left(V^{(\ell)}\right)\right)\right) \\
& \times\left(\prod _ { \ell = 1 } ^ { m } \left(\frac{\Delta\left(U^{(l)} ; V^{(\ell-1)}\right) \Delta\left(V^{(l)} ; U^{(\ell-1)}\right) \mathrm{e}^{-h\left(V^{(\ell)}, z_{\ell-1}\right)-h\left(V^{(l-1)}, z_{\ell}\right)}}{\left.\left.\Delta\left(U^{(l)} ; U^{(\ell-1)}\right) \Delta\left(V^{(l)} ; V^{(\ell-1)}\right) \mathrm{e}^{h\left(U^{(\ell)}, z_{\ell-1}\right)+h\left(U^{(\ell-1)}, z_{\ell}\right)}\right)\right),}\right.\right.
\end{aligned}
$$

with $\left|z_{m}\right|<\ldots<\left|z_{1}\right|<1, \nu_{m+1}=-\infty, x_{0}=x_{m+1}=t_{0}=t_{m+1}=u_{0}=u_{m+1}=$ 0 . In terms of $z_{\ell}=-\mathrm{e}^{\nu_{\ell}}, \operatorname{Re} \nu_{\ell}<0,-\pi<\operatorname{Im} \nu_{\ell}<\pi$, one has $A_{1}\left(z_{\ell}\right)=\chi_{\emptyset}^{\prime}\left(\nu_{\ell}\right)$ and $A_{2}\left(z_{\ell}\right)=\chi_{\emptyset}\left(\nu_{\ell}\right)$ with $\chi_{\emptyset}$ given by (56) and $B\left(z_{\ell_{1}}, z_{\ell_{2}}\right)=K_{\emptyset, \emptyset}\left(\nu_{\ell_{1}}, \nu_{\ell_{2}}\right)$ with $K_{\emptyset, \emptyset}$ given by (101). The $n_{\ell}$-uples $U^{(\ell)}, V^{(\ell)}$ are defined in terms of the $n_{\ell}$-uples $\mathcal{P}_{\ell}, \mathcal{H}_{\ell}$ as $U^{(\ell)}=\left(\kappa_{a}\left(\nu_{\ell}\right), a \in \mathcal{P}_{\ell}\right), V^{(\ell)}=\left(-\kappa_{a}\left(\nu_{\ell}\right), a \in \mathcal{H}_{\ell}\right)$. The quantities $\Delta(W), \Delta\left(W ; W^{\prime}\right)$ for tuples $W=\left(w_{1}, \ldots, w_{n}\right), W^{\prime}=\left(w_{1}^{\prime}, \ldots, w_{n^{\prime}}^{\prime}\right)$ are defined as $\Delta(W)=\prod_{1 \leq i<j \leq n}\left(w_{j}-w_{i}\right)$, $\Delta\left(W ; W^{\prime}\right)=\prod_{i=1}^{n} \prod_{i^{\prime}=1}^{n^{\prime}}\left(w_{i}-w_{i^{\prime}}^{\prime}\right)$. The remaining factors are given by $h\left(U^{\left(\ell_{1}\right)}, z_{\ell_{2}}\right)=$ $\sum_{a \in \mathcal{P}_{\ell_{1}}} \int_{-\infty}^{0} \mathrm{~d} u \chi_{\emptyset}^{\prime \prime}\left(u+\nu_{\ell_{2}}\right) / \kappa_{a}\left(u+\nu_{\ell_{1}}\right), h\left(V^{\left(\ell_{1}\right)}, z_{\ell_{2}}\right)=\sum_{a \in \mathcal{H}_{\ell_{1}}} \int_{-\infty}^{0} \mathrm{~d} u \chi_{\emptyset}^{\prime \prime}\left(u+\nu_{\ell_{2}}\right) / \kappa_{a}(u+$ $\left.\nu_{\ell_{1}}\right)$ and

$$
\begin{aligned}
& \hat{f}_{\ell}\left(U^{(\ell)}\right)=(-1)^{n_{\ell}} \mathrm{e}^{2 \sum_{a \in P_{\ell}} \int_{-\infty}^{\nu_{\ell}} \mathrm{d} v \frac{\chi_{\emptyset}^{\prime \prime}(v)}{\kappa_{a}(v)}} \prod_{a \in P_{\ell}} \frac{\mathrm{e}^{\left(t_{\ell}-t_{\ell-1}\right) \frac{\kappa_{a}\left(\nu_{\ell}\right)^{3}}{3}+\left(x_{\ell}-x_{\ell-1}\right) \frac{\kappa_{a}\left(\nu_{\ell}\right)^{2}}{2}+\left(u_{\ell}-u_{\ell-1}\right) \kappa_{a}\left(\nu_{\ell}\right)}}{\kappa_{a}\left(\nu_{\ell}\right)} \\
& \hat{f}_{\ell}\left(V^{(\ell)}\right)=\mathrm{e}^{2 \sum_{a \in H_{\ell}} \int_{-\infty}^{\nu_{\ell}} \mathrm{d} v \frac{\chi_{\emptyset}^{\prime \prime}(v)}{\kappa_{a}(v)}} \prod_{a \in H_{\ell}} \frac{\mathrm{e}^{\left(t_{\ell}-t_{\ell-1}\right) \frac{\kappa_{a}\left(\nu_{\ell}\right)^{3}}{3}-\left(x_{\ell}-x_{\ell-1}\right) \frac{\kappa_{a}\left(\nu_{\ell}\right)^{2}}{2}+\left(u_{\ell}-u_{\ell-1}\right) \kappa_{a}\left(\nu_{\ell}\right)}}{\kappa_{a}\left(\nu_{\ell}\right)} \cdot(153)
\end{aligned}
$$

Because of the Vandermonde determinants $\Delta\left(U^{(\ell)}\right), \Delta\left(V^{(\ell)}\right)$, only tuples $\mathcal{P}_{\ell}, \mathcal{H}_{\ell}$ with distinct elements contribute to 152 . Since the summand is invariant under permutations of the elements of $\mathcal{P}_{\ell}, \mathcal{H}_{\ell}$, one can sum over subsets $P_{\ell}, H_{\ell}$ of $\mathbb{Z}+1 / 2$ instead, up to a factor $\prod_{\ell=1}^{m}\left(n_{\ell} !\right)^{2}$ counting the number of permutations. Making the changes of variables $z_{\ell}=-\mathrm{e}^{\nu_{\ell}}$, one finds after some simplifications

$$
\begin{gathered}
F(\vec{t}, \vec{x}, \vec{u})=\int_{c_{1}-\mathrm{i} \pi}^{c_{1}+\mathrm{i} \pi} \frac{\mathrm{d} \nu_{1}}{2 \mathrm{i} \pi} \ldots \int_{c_{m}-\mathrm{i} \pi}^{c_{m}+\mathrm{i} \pi} \frac{\mathrm{d} \nu_{m}}{2 \mathrm{i} \pi}\left(\prod_{\ell=1}^{m} \sum_{\substack{P_{\ell}, H_{\ell} \subset \mathbb{Z}+1 / 2 \\
\left|P_{\ell}\right|=\left|H_{\ell}\right|}}\right) \\
\times\left(\prod _ { \ell = 1 } ^ { m } \left((\mathrm{i} / 4)^{2 n_{\ell}} V_{P_{\ell}}^{2} V_{H_{\ell}}^{2} \mathrm{e}^{2 \mathrm{i} \pi\left(x_{\ell}-x_{\ell-1}\right)\left(\sum_{a \in P_{\ell}} a-\sum_{a \in H_{\ell}} a\right)}\right.\right. \\
\times \mathrm{e}^{\left.\left.\left(t_{\ell}-t_{\ell-1}\right) \chi_{P_{\ell}\left(\nu_{\ell}\right)-\left(u_{\ell}-u_{\ell-1}\right) \chi_{P_{\ell}}^{\Delta_{\ell}}\left(\nu_{\ell}\right)+2 J_{P_{\ell}}^{\Delta}\left(\nu_{\ell}\right)}^{\Delta_{\ell}}\right)\right)} \\
\times \prod_{\ell=1}^{m-1} \frac{\left(1-\mathrm{e}^{\nu_{\ell+1}-\nu_{\ell}}\right)^{-1+n_{\ell}}\left(1-\mathrm{e}^{\nu_{\ell}-\nu_{\ell+1}}\right)^{n_{\ell+1}} \mathrm{e}^{-2 K_{P_{\ell}, P_{\ell+1}}^{\Delta_{\ell}, \Delta_{\ell+1}}\left(\nu_{\ell}, \nu_{\ell+1}\right)}}{\left(\prod_{a \in P_{\ell}} \prod_{b \in P_{\ell+1}}\left(\frac{2 \mathrm{i} \pi b-\nu_{\ell+1}}{4}-\frac{2 \mathrm{i} \pi a-\nu_{\ell}}{4}\right)\right)\left(\prod_{a \in H_{\ell}} \prod_{b \in H_{\ell+1}}\left(\frac{2 \mathrm{i} \pi b-\nu_{\ell+1}}{4}-\frac{2 \mathrm{i} \pi a-\nu_{\ell}}{4}\right)\right)}
\end{gathered}
$$

with $c_{m}<\ldots<c_{1}<0, n_{\ell}=\left|P_{\ell}\right|=\left|H_{\ell}\right|$ and $\Delta_{\ell}=P_{\ell} \ominus H_{\ell}$. 


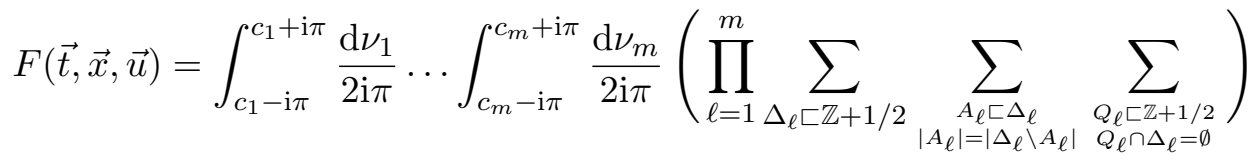

$$
\begin{aligned}
& \times\left(\prod _ { \ell = 1 } ^ { m } \left((\mathrm{i} / 4)^{\left|\Delta_{\ell}\right|} V_{A_{\ell}}^{2} V_{\Delta_{\ell} \backslash A_{\ell}}^{2} \mathrm{e}^{2 \mathrm{i} \pi\left(x_{\ell}-x_{\ell-1}\right)\left(\sum_{a \in A_{\ell}} a-\sum_{a \in \Delta_{\ell} \backslash A_{\ell}} a\right)}\right.\right.
\end{aligned}
$$

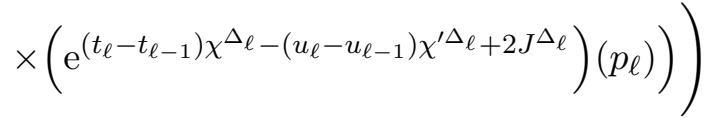

$$
\begin{aligned}
& \times\left(\prod _ { \ell = 1 } ^ { m - 1 } \left(\frac{\left(1-\mathrm{e}^{\nu_{\ell+1}-\nu_{\ell}}\right)^{\left|\Delta_{\ell}\right| / 2}\left(1-\mathrm{e}^{\nu_{\ell}-\nu_{\ell+1}}\right)^{\left|\Delta_{\ell+1}\right| / 2}}{1-\mathrm{e}^{\nu_{\ell+1}-\nu_{\ell}}}\right.\right. \\
& \left.\left.\times \frac{\mathrm{e}^{-2 K^{\Delta_{\ell}, \Delta_{\ell+1}}\left(p_{\ell}, p_{\ell+1}\right)}}{\prod_{a \in \Delta_{\ell}} \prod_{b \in \Delta_{\ell+1}}\left(\frac{2 \mathrm{i} \pi b-\nu_{\ell+1}}{4}-\frac{2 \mathrm{i} \pi a-\nu_{\ell}}{4}\right) \frac{1+\sigma_{a}\left(A_{\ell}\right) \sigma_{b}\left(A_{\ell+1}\right)}{2}}\right)\right),
\end{aligned}
$$

1610 where $p_{\ell}=\left[\nu_{\ell}, Q_{\ell}\right]$. This is essentially (13). 


\subsubsection{Pole structure of $(16)$}

The integrand in (16) has potential poles and zeroes at $\nu_{\ell+1}=\nu_{\ell}+2 \mathrm{i} \pi m, m \in \mathbb{Z}$. More precisely defining from (14), (15) and (118) the integer

$$
\begin{aligned}
\alpha_{\ell}=-1 & +\left(\frac{\left|\Delta_{\ell}\right|}{2}+\frac{\left|\Delta_{\ell+1}\right|}{2}-\left|A_{\ell} \cap\left(A_{\ell+1}-m\right)\right|-\left|\left(\Delta_{\ell} \backslash A_{\ell}\right) \cap\left(\left(\Delta_{\ell+1} \backslash A_{\ell+1}\right)-m\right)\right|\right) \\
+\left(\left|P_{\ell}\right|+\left|P_{\ell+1}\right|+\left|\Delta_{\ell} \cap\left(\Delta_{\ell+1}-m\right)\right|\right. & \\
& \left.-\left|P_{\ell} \cap\left(P_{\ell+1}-m\right)\right|-\left|\left(P_{\ell} \cup \Delta_{\ell}\right) \cap\left(\left(P_{\ell+1} \cup \Delta_{\ell+1}\right)-m\right)\right|\right)
\end{aligned}
$$

with $P_{\ell} \cap \Delta_{\ell}=\emptyset, A_{\ell} \subset \Delta_{\ell}$ and $\left|A_{\ell}\right|=\left|\Delta_{\ell}\right| / 2$ as in (13), the integrand has at the point $\nu_{\ell+1}=\nu_{\ell}+2 \mathrm{i} \pi m$ a zero of order $\alpha_{\ell}$ if $\alpha_{\ell}>0$ and a pole of order $-\alpha_{\ell}$ if $\alpha_{\ell}<0$. We show below that for any choice of the integer $m$ and of the sets $\Delta_{\ell}, A_{\ell}, P_{\ell}, \Delta_{\ell+1}, A_{\ell+1}, P_{\ell+1}$ as in (13), one has $\alpha_{\ell} \geq-1$, with $\alpha_{\ell}=-1$ if and only if $\Delta_{\ell+1}=\Delta_{\ell}+m, A_{\ell+1}=A_{\ell}+m$ and $P_{\ell+1}=P_{\ell}+m$.

We consider first the terms of (159) in the first line within the parenthesis. Using $\left|A_{\ell} \cap\left(A_{\ell+1}-m\right)\right| \leq \min \left(\left|A_{\ell}\right|,\left|A_{\ell+1}\right|\right)=\min \left(\left|\Delta_{\ell}\right|,\left|\Delta_{\ell+1}\right|\right) / 2$, and similarly for $\mid\left(\Delta_{\ell} \backslash A_{\ell}\right) \cap$ $\left(\left(\Delta_{\ell+1} \backslash A_{\ell+1}\right)-m\right) \mid$, the first parenthesis of $(159)$ is non-negative, and equal to zero if and only if $\Delta_{\ell+1}=\Delta_{\ell}+m$ and $A_{\ell+1}=A_{\ell}+m$.

We consider then the terms in the second and third line of (159). After some manipulations, we observe that the sum of these terms is equal to $\left|P_{\ell}\right|-\mid P_{\ell} \cap\left(\left(P_{\ell+1}-m\right) \cup\left(\Delta_{\ell+1}-\right.\right.$ $m))|+| P_{\ell+1}|-|\left(P_{\ell} \cup \Delta_{\ell}\right) \cap\left(P_{\ell+1}-m\right) \mid$, which is manifestly non-negative. The integrand in (13) may thus have a pole only if both parentheses in (159) are equal to zero. Provided that $\Delta_{\ell+1}=\Delta_{\ell}+m$, the second parenthesis is equal to zero if and only if $P_{\ell+1}=P_{\ell}+m$, which concludes the proof.

\section{A Derivation of the identity $(76)$}

In this appendix, we derive the identity $\sqrt{76}$ for the integral $f_{\beta_{n} \cdot P} \chi^{\prime \prime}(v)^{2} \mathrm{~d} v, n \in \mathbb{Z}$. The identity is obviously true for $n=0$ since $\beta_{0} \cdot P$ is homotopic to an empty loop. We consider first the case $n=1$, for which a detailed calculation is needed, and then generalize to arbitrary $n \in \mathbb{Z}$ by using translation properties of the functions $J_{P}$.

\section{A.1 Case $n=1$}

We introduce positive numbers $\epsilon, \delta, 0<\epsilon \ll \delta \ll 1$. By definition of the path $\beta_{1} \cdot P$, one has

$$
f_{\beta_{1} \cdot P} \chi^{\prime \prime}(v)^{2} \mathrm{~d} v=f_{-\infty}^{\mathrm{i}(\pi+\delta)-\epsilon} \mathrm{d} v \chi_{P}^{\prime \prime}(v)^{2}-f_{-\infty}^{\mathrm{i}(\pi+\delta)+\epsilon} \mathrm{d} v \chi_{P \ominus\{1 / 2\}}^{\prime \prime}(v)^{2},
$$

with both paths of integration contained in $\mathbb{D}$ on the right hand side. The path for the second integral on the right has to cross the imaginary axis in the interval $-\pi<\operatorname{Im} v<\pi$, see figure 20. At this point, $\delta$ need not be infinitesimal (we only require that $0<\delta<2 \pi$ ), but it will be convenient in the following in order to compute some integrals by expanding close to the singularity at $v=\mathrm{i} \pi$.

From (64), 63), (50) and $\kappa_{b}^{\prime \prime}=3 / \kappa_{b}$, the function $\chi_{P}^{\prime \prime}(v)$ verifies

$$
\chi_{P}^{\prime \prime}(v)=\chi_{\emptyset}^{\prime \prime}(v)+\sum_{b \in P} \frac{2}{\kappa_{b}(v)}
$$


1644

and

$$
\chi_{P}^{\prime \prime}(v)=\lim _{M \rightarrow \infty}\left(\sqrt{\frac{2 M}{\pi}}-\sum_{b=-M+1 / 2}^{M-1 / 2} \frac{\sigma_{b}(P)}{\kappa_{b}(v)}\right) .
$$

1645 1646

1647

1648

1649

1650

1651

1652

1653

1654 1655

1656

Since $\sigma_{1 / 2}(P)=-\sigma_{1 / 2}(P \ominus\{1 / 2\})$, the function $\chi_{P}^{\prime \prime}(v)+\sigma_{1 / 2}(P) / \kappa_{1 / 2}(v)=\chi_{P \ominus\{1 / 2\}}^{\prime \prime}(v)-$ $\sigma_{1 / 2}(P) / \kappa_{1 / 2}(v)$ does not have a branch point at $v=\mathrm{i} \pi$, so that

$$
f_{-\infty}^{\mathrm{i}(\pi+\delta)-\epsilon} \mathrm{d} v\left(\chi_{P}^{\prime \prime}(v)+\frac{\sigma_{1 / 2}(P)}{\kappa_{1 / 2}(v)}\right)^{2}-f_{-\infty}^{\mathrm{i}(\pi+\delta)+\epsilon} \mathrm{d} v\left(\chi_{P \ominus\{1 / 2\}}^{\prime \prime}(v)-\frac{\sigma_{1 / 2}(P)}{\kappa_{1 / 2}(v)}\right)^{2}=0 .
$$

This leads to

$$
\begin{aligned}
f_{\beta_{1} \cdot P} \chi^{\prime \prime}(v)^{2} \mathrm{~d} v= & f_{-\infty}^{\mathrm{i}(\pi+\delta)-\epsilon} \mathrm{d} v\left(-\frac{1}{\kappa_{1 / 2}(v)^{2}}-2 \sigma_{1 / 2}(P) \frac{\chi_{P}^{\prime \prime}(v)}{\kappa_{1 / 2}(v)}\right) \\
& -f_{-\infty}^{\mathrm{i}(\pi+\delta)+\epsilon} \mathrm{d} v\left(-\frac{1}{\kappa_{1 / 2}(v)^{2}}+2 \sigma_{1 / 2}(P) \frac{\chi_{P \ominus\{1 / 2\}}^{\prime \prime}(v)}{\kappa_{1 / 2}(v)}\right),
\end{aligned}
$$

which, using (161), gives

$$
\begin{gathered}
f_{\beta_{1} \cdot P} \chi^{\prime \prime}(v)^{2} \mathrm{~d} v=\sigma_{1 / 2}(P) f_{-\infty}^{\mathrm{i}(\pi+\delta)-\epsilon} \mathrm{d} v\left(\frac{\sigma_{1 / 2}(P)-2}{\kappa_{1 / 2}(v)^{2}}-\frac{2 \chi_{\emptyset}^{\prime \prime}(v)}{\kappa_{1 / 2}(v)}-\sum_{b \in P \backslash\{1 / 2\}} \frac{4}{\kappa_{1 / 2}(v) \kappa_{b}(v)}\right) \\
-\sigma_{1 / 2}(P) f_{-\infty}^{\mathrm{i}(\pi+\delta)+\epsilon} \mathrm{d} v\left(\frac{\sigma_{1 / 2}(P)+2}{\kappa_{1 / 2}(v)^{2}}+\frac{2 \chi_{\emptyset}^{\prime \prime}(v)}{\kappa_{1 / 2}(v)}+\sum_{b \in P \backslash\{1 / 2\}} \frac{4}{\kappa_{1 / 2}(v) \kappa_{b}(v)}\right) \cdot(165)
\end{gathered}
$$

The integrals needed are computed in appendix C. Using (184) and (185), we obtain after some simplifications

$$
\begin{aligned}
\frac{1}{2} f_{\beta_{1} \cdot P} \chi^{\prime \prime}(v)^{2} \mathrm{~d} v=\mathrm{i} \pi+\sigma_{1 / 2}(P)\left(\mathrm { i } \pi \left(|P|_{+}\right.\right. & \left.-|P|_{-}-1 / 2\right)-2 \log 2 \\
& \left.+\sum_{b \in P \backslash\{1 / 2\}} \log \frac{\pi^{2}(b-1 / 2)^{2}}{4}\right),
\end{aligned}
$$

which is equivalent to 76 with $n=1$.

\section{A.2 Extension to $n>1$}

For $n \geq 2$, we write the telescopic sum

$$
\begin{aligned}
J_{P-n}(\nu-2 \mathrm{i} \pi n)-J_{P \ominus B_{n}}(\nu)=\sum_{m=1}^{n} & \left(J_{(P-m) \ominus B_{n-m}}(\nu-2 \mathrm{i} \pi m)\right. \\
& \left.-J_{(P-m+1) \ominus B_{n-m+1}}(\nu-2 \mathrm{i} \pi(m-1))\right) .
\end{aligned}
$$

Noting that $(P-m+1) \ominus B_{n-m+1}=\left((P-m) \ominus B_{n-m}+1\right) \ominus\{1 / 2\}$, see the group identity (36), one has from (75)

$$
\frac{1}{2} f_{\beta_{n} \cdot P} \chi^{\prime \prime}(v)^{2} \mathrm{~d} v=\frac{1}{2} \sum_{m=1}^{n} f_{\beta_{1} \cdot\left((P-m) \ominus B_{n-m}+1\right)} \chi^{\prime \prime}(v)^{2} \mathrm{~d} v .
$$

Using the identity (76) with $n=1$ derived previously, we arrive at

$$
\frac{1}{2} f_{\beta_{n} \cdot P} \chi^{\prime \prime}(v)^{2} \mathrm{~d} v=\frac{1}{2} \sum_{m=1}^{n}\left(W_{(P-m+1) \ominus B_{n-m+1}}-W_{(P-m) \ominus B_{n-m}}\right),
$$

whose right hand side telescopically reduces to $\left(W_{P \ominus B_{n}}-W_{P-n}\right) / 2$. This proves $(76)$ for $n \geq 2$. 


\section{A.3 Extension to $n<0$}

Let $n$ be a positive integer. The replacements $\nu \rightarrow \nu+2 \mathrm{i} \pi n$ and $P \rightarrow\left(P \ominus B_{n}\right)-n$ in (75) give

$$
J_{P \ominus B_{n}-n}(\nu)=J_{P}(\nu+2 \mathrm{i} \pi n)+\frac{1}{2} f_{\beta_{n} \cdot\left(P \ominus B_{n}\right)} \chi^{\prime \prime}(v)^{2} \mathrm{~d} v .
$$

Changing the order of the terms and using $P \ominus B_{n}-n=(P-n) \ominus B_{-n}$, one has

$$
J_{P}(\nu+2 \mathrm{i} \pi n)=J_{(P-n) \ominus B_{-n}}(\nu)-\frac{1}{2} f_{\beta_{n} \cdot\left(P \ominus B_{n}\right)} \chi^{\prime \prime}(v)^{2} \mathrm{~d} v,
$$

which, from 75 with $n$ replaced by $-n$, gives

$$
\frac{1}{2} f_{\beta_{-n} \cdot(P-n)} \chi^{\prime \prime}(v)^{2} \mathrm{~d} v=-\frac{1}{2} f_{\beta_{n} \cdot\left(P \ominus B_{n}\right)} \chi^{\prime \prime}(v)^{2} \mathrm{~d} v .
$$

Using the identity (76) for $n>0$ derived previously, this leads to

$$
\frac{1}{2} f_{\beta_{-n} \cdot(P-n)} \chi^{\prime \prime}(v)^{2} \mathrm{~d} v=-W_{P}+W_{P \ominus B_{n}-n} .
$$

Replacing $P$ by $P+n$ finally leads to $(76)$ with $n$ replaced by $-n<0$.

\section{B Derivation of the identities $(106)$ and $(107)$}

In this appendix, we derive the identities (106) and (107) for some integrals over the paths $\gamma_{n, 0}$ and $\gamma_{0, m}$. The identities are obviously true for $n=0$ or $m=0$ since the paths are then homotopic to empty loops. We start with the identity (106), which is proved first for $n=1$, where a detailed calculation is needed, and then generalized to arbitrary $n \in \mathbb{Z}$ by using translation properties of the functions $K_{P, Q}$. The identity (107) is then obtained by exchanging $\mu$ with $\nu$ and $P$ with $Q$.

\section{B.1 Case $n=1$ for 106}

The integral $f_{\gamma_{1,0}} \mathrm{~d} u \mathcal{A}_{u}\left(\chi_{P}^{\prime \prime}(\cdot+\nu) \chi_{Q}^{\prime \prime}(\cdot+\mu)\right)$ is equal to zero if $\operatorname{Re} \nu<0$ since the path $\gamma_{1,0}$ is empty then. Therefore, we restrict to $\operatorname{Re} \nu>0$ in the rest of this section. We introduce positive numbers $\epsilon, \delta, 0<\epsilon \ll \delta \ll 1$. We want to compute the integral

$$
\begin{aligned}
& f_{\gamma_{1,0}} \mathrm{~d} u \mathcal{A}_{u}\left(\chi_{P}^{\prime \prime}(\cdot+\nu) \chi_{Q}^{\prime \prime}(\cdot+\mu)\right) \\
& =f_{-\infty}^{\mathrm{i}(\pi+\delta)-\nu-\epsilon} \mathrm{d} u \chi_{P}^{\prime \prime}(u+\nu) \chi_{Q}^{\prime \prime}(u+\mu)-f_{-\infty}^{\mathrm{i}(\pi+\delta)-\nu+\epsilon} \mathrm{d} u \chi_{P \ominus\{1 / 2\}}^{\prime \prime}(u+\nu) \chi_{Q}^{\prime \prime}(u+\mu),
\end{aligned}
$$

where the paths of integration in the second line are contained in $\mathbb{D}$. The path for the last integral in the second line has to cross the imaginary axis in the interval $-\pi<\operatorname{Im} v<\pi$, see figure 20. Additionally, if $\operatorname{Re} \mu>\operatorname{Re} \nu$, the paths of both integrals in the second line must cross the line $\operatorname{Re}(u+\mu)=0$ in the interval $-\pi<\operatorname{Im}(u+\mu)<\pi$. At this point, $\delta$ need not be infinitesimal (we only require that $0<\delta<2 \pi$ ), but it will be convenient in the following in order to compute some integrals by expanding close to the singularity at $u=\mathrm{i} \pi-\nu$. 
Because of 162, the function $\chi_{P}^{\prime \prime}(v)+\sigma_{1 / 2}(P) / \kappa_{1 / 2}(v)=\chi_{P \ominus\{1 / 2\}}^{\prime \prime}(v)-\sigma_{1 / 2}(P) / \kappa_{1 / 2}(v)$ does not have a branch point at $v=\mathrm{i} \pi$, so that

$$
\begin{aligned}
\int_{-\infty}^{\mathrm{i}(\pi+\delta)-\nu-\epsilon} \mathrm{d} u & \left(\chi_{P}^{\prime \prime}(u+\nu)+\frac{\sigma_{1 / 2}(P)}{\kappa_{1 / 2}(u+\nu)}\right) \chi_{Q}^{\prime \prime}(u+\mu) \\
= & f_{-\infty}^{\mathrm{i}(\pi+\delta)-\nu+\epsilon} \mathrm{d} u\left(\chi_{P}^{\prime \prime}(u+\nu)-\frac{\sigma_{1 / 2}(P)}{\kappa_{1 / 2}(u+\nu)}\right) \chi_{Q}^{\prime \prime}(u+\mu)=0 .
\end{aligned}
$$

This leads to

$$
\begin{aligned}
& f_{\gamma_{1,0}} \mathrm{~d} u \mathcal{A}_{u}\left(\chi_{P}^{\prime \prime}(\cdot+\nu) \chi_{Q}^{\prime \prime}(\cdot+\mu)\right) \\
& =-\sigma_{1 / 2}(P)\left(f_{-\infty}^{\mathrm{i}(\pi+\delta)-\nu-\epsilon} \mathrm{d} u \frac{\chi_{Q}^{\prime \prime}(u+\mu)}{\kappa_{1 / 2}(u+\nu)}+f_{-\infty}^{\mathrm{i}(\pi+\delta)-\nu+\epsilon} \mathrm{d} u \frac{\chi_{Q}^{\prime \prime}(u+\mu)}{\kappa_{1 / 2}(u+\nu)}\right) .
\end{aligned}
$$

Using (161), one has

$$
\begin{aligned}
& f_{\gamma_{1,0}} \mathrm{~d} u \mathcal{A}_{u}\left(\chi_{P}^{\prime \prime}(\cdot+\nu) \chi_{Q}^{\prime \prime}(\cdot+\mu)\right) \\
& =-\sigma_{1 / 2}(P)\left(\int_{-\infty}^{\mathrm{i}(\pi+\delta)-\nu-\epsilon} \mathrm{d} u \frac{\chi_{\emptyset}^{\prime \prime}(u+\mu)}{\kappa_{1 / 2}(u+\nu)}+\int_{-\infty}^{\mathrm{i}(\pi+\delta)-\nu+\epsilon} \mathrm{d} u \frac{\chi_{\emptyset}^{\prime \prime}(u+\mu)}{\kappa_{1 / 2}(u+\nu)}\right. \\
& \left.\quad+2 \sum_{b \in Q}\left(f_{-\infty}^{\mathrm{i}(\pi+\delta)-\nu-\epsilon} \frac{\mathrm{d} u}{\kappa_{1 / 2}(u+\nu) \kappa_{b}(u+\mu)}+f_{-\infty}^{\mathrm{i}(\pi+\delta)-\nu+\epsilon} \frac{\mathrm{d} u}{\kappa_{1 / 2}(u+\nu) \kappa_{b}(u+\mu)}\right)\right) .
\end{aligned}
$$

The remaining integrals are computed in appendix D. Using (194) and (201), we find

$$
\frac{1}{2} f_{\gamma_{1,0}} \mathrm{~d} u \mathcal{A}_{u}\left(\chi_{P}^{\prime \prime}(\cdot+\nu) \chi_{Q}^{\prime \prime}(\cdot+\mu)\right)=2 \sigma_{1 / 2}(P)\left(I_{0}(\mu-\nu+\mathrm{i} \pi)+\sum_{b \in Q} \log \frac{\kappa_{b}(\mu-\nu+\mathrm{i} \pi)}{\sqrt{8}}\right) .
$$

Noting from 103 that $W_{P \ominus\{1 / 2\}, Q}(z)-W_{P, Q}(z)=2 \sigma_{1 / 2}(P)\left(I_{0}(z)+\sum_{b \in Q} \log \frac{\kappa_{b}(z)}{\sqrt{8}}\right)$ and from (104) that $Z_{P \ominus\{1 / 2\}, Q}-Z_{P, Q}=0$, we finally obtain (106) for $n=1$ after replacing $P$ by $P+1$ in 178$)$.

\section{B.2 Extension to $n>1$ and $n<0$}

The extension of (106) from $n=1$ to all $n \in \mathbb{Z}$ works essentially the same as in appendix A

\section{B.3 Proof of (107)}

Exchanging $\nu$ with $\mu$ and replacing $n$ by $m$ transforms the path $\gamma_{n, 0}$ to $\gamma_{0, m}$. Replacing also $P$ with $Q$ in 106 gives

$$
\begin{aligned}
& \frac{1}{2} f_{\gamma_{0, m}} \mathrm{~d} u \mathcal{A}_{u}\left(\chi_{Q+m}^{\prime \prime}(\cdot+\mu) \chi_{P}^{\prime \prime}(\cdot+\nu)\right) \\
& =1_{\{\operatorname{Re} \mu>0\}}\left(W_{(Q+m) \ominus B_{m}, P}(\nu-\mu+\mathrm{i} \pi)-W_{Q+m, P}(\nu-\mu+\mathrm{i} \pi)\right. \\
& \left.\quad+1_{\{\operatorname{Re} \nu>\operatorname{Re} \mu\}}\left(Z_{(Q+m) \ominus B_{m}, P}-Z_{Q+m}, P\right)\right) .
\end{aligned}
$$

Using (105) then leads to (107). 


\section{Calculations of some integrals between $-\infty$ and $\nu \in \mathbb{D}$}

In this appendix, we compute some integrals between $-\infty$ and $\nu \in \mathbb{D}$, with a path of integration contained in $\mathbb{D}$. For some integrals, indicated by the symbol $f$, a regularization at $-\infty$ is needed due to the presence of logarithmic divergences.

\section{C.1 The functions $\log \kappa_{a}$ and $\log \left(\kappa_{a}+\kappa_{b}\right)$ are analytic in $\mathbb{D}$}

For any $a \in \mathbb{Z}+1 / 2$, the function $\kappa_{a}$ defined in $(50)$ is analytic in $\mathbb{D}$. When $\operatorname{Re} v=0$, $-\pi<\operatorname{Im} v<\pi$ and $\arg \left(\kappa_{a}(v)\right)=\operatorname{sgn}(a) \pi / 4$. Otherwise, $\operatorname{Re} v$ is non-zero and one has

$$
\arg \left(\kappa_{a}(v)\right) \in\left\{\begin{array}{cc}
(-3 \pi / 4,-\pi / 4) & \operatorname{Re} v>0 \text { and } a<0 \\
(\pi / 4,3 \pi / 4) & \operatorname{Re} v>0 \text { and } a>0 \\
(-\pi / 4, \pi / 4) & \operatorname{Re} v<0
\end{array}\right.
$$

which implies in particular that $\log \kappa_{a}$ is analytic in $\mathbb{D}$ if the branch cut of the logarithm is chosen as $\mathbb{R}^{-}$.

For $a, b \in \mathbb{Z}+1 / 2$ and $v \in \mathbb{D}$, the discussion above imply constraints on the argument of $\kappa_{a}(v)+\kappa_{b}(v), a, b \in \mathbb{Z}+1 / 2$. When $\operatorname{Re} v=0,-\pi<\operatorname{Im} v<\pi$, one has $\arg \left(\kappa_{a}(v)+\kappa_{b}(v)\right)=$ $(\operatorname{sgn}(a)+\operatorname{sgn}(b)) \mathrm{i} \pi / 4$. Otherwise, $\operatorname{Re} v$ is non-zero and $(180)$ implies

$$
\arg \left(\kappa_{a}(v)+\kappa_{b}(v)\right) \in\left\{\begin{array}{ll}
(-3 \pi / 4,-\pi / 4) & \operatorname{Re} v>0, a<0 \text { and } b<0 \\
(\pi / 4,3 \pi / 4) & \operatorname{Re} v>0, a>0 \text { and } b>0 \\
(-\pi / 4, \pi / 4) & \operatorname{Re} v>0, a b<0 \\
(-\pi / 4, \pi / 4) & \operatorname{Re} v<0
\end{array},\right.
$$

In the case $\operatorname{Re} v>0, a b<0$, we have used $\kappa_{a}(v)+\kappa_{b}(v)=4 \mathrm{i} \pi(a-b) /\left(\kappa_{a}(v)-\kappa_{b}(v)\right)$. We observe in particular from (181) that $\arg \left(\kappa_{a}(v)+\kappa_{b}(v)\right)$ always stays between $-3 \pi / 4$ and $3 \pi / 4$, and thus $\kappa_{a}(v)+\kappa_{b}(v)$ never reaches the negative real axis $\mathbb{R}^{-}$. The function $\log \left(\kappa_{a}(v)+\kappa_{b}(v)\right)$ is thus analytic in $\mathbb{D}$ for any $a, b \in \mathbb{Z}+1 / 2$ with the branch cut of the logarithm chosen as $\mathbb{R}^{-}$.

\section{C.2 Integral of $\kappa_{a}(v)^{-1} \kappa_{b}(v)^{-1}$}

Let $a, b \in \mathbb{Z}+1 / 2$ and $\nu \in \mathbb{D}$. We consider the integral $\int_{-\Lambda}^{\nu} \mathrm{d} v \kappa_{a}(v)^{-1} \kappa_{b}(v)^{-1}$ with a path of integration staying in $\mathbb{D}$ and $|\Lambda| \rightarrow \infty, \operatorname{Re} \Lambda>0$. The identity

$$
\frac{1}{\kappa_{a}(v) \kappa_{b}(v)}=-\partial_{v} \log \left(\kappa_{a}(v)+\kappa_{b}(v)\right)
$$

allows to compute the integral explicitly since $\log \left(\kappa_{a}(v)+\kappa_{b}(v)\right)$ is analytic in $\mathbb{D}$ when the branch cut of the logarithm is chosen as $\mathbb{R}^{-}$, as showed in appendix C.1. Taking $|\Lambda| \rightarrow \infty$, $\operatorname{Re} \Lambda>0$, one finds

$$
\int_{-\Lambda}^{\nu} \frac{\mathrm{d} v}{\kappa_{a}(v) \kappa_{b}(v)} \simeq \log \sqrt{8 \Lambda}-\log \left(\kappa_{a}(\nu)+\kappa_{b}(\nu)\right)
$$

For any $\nu \in \mathbb{D}$, the regularized integral is then equal to

$$
f_{-\infty}^{\nu} \frac{\mathrm{d} v}{\kappa_{a}(v) \kappa_{b}(v)}=-\log \left(\frac{\kappa_{a}(\nu)+\kappa_{b}(\nu)}{\sqrt{8}}\right) .
$$




\section{C.3 Integral of $\chi_{\emptyset}^{\prime \prime}(v) / \kappa_{a}(v)$ between $-\infty$ and a branch point}

Let $\delta$ be a positive real number and $\theta \neq 0,-\pi<\theta<\pi$. In the limit $\delta \rightarrow 0$ one has

$$
\int_{-\infty}^{\mathrm{i}\left(2 \pi a+\mathrm{e}^{\mathrm{i} \theta} \delta\right)} \mathrm{d} v \frac{\chi_{\emptyset}^{\prime \prime}(v)}{\kappa_{a}(v)} \simeq \log \sqrt{4 \delta}+\frac{\mathrm{i} \theta}{2}-\operatorname{sgn}(\theta) \frac{\mathrm{i} \pi}{4}+1_{\{\theta<0\}} \mathrm{i} \pi a,
$$

which can be derived by taking $\mu=\nu=0$ and $v=\mathrm{i}\left(2 \pi a+\mathrm{e}^{\mathrm{i} \theta} \delta\right)$ in 200$)$, after rather tedious simplifications using $\kappa_{a}\left(\mathrm{i}\left(2 \pi a+\mathrm{e}^{\mathrm{i} \theta} \delta\right)\right)=\mathrm{e}^{\mathrm{i} \theta}-\left(1-41_{\{\theta<0\}} 1_{\{a>0\}}\right) \frac{\mathrm{i} \pi}{4} \sqrt{2 \delta}, \kappa_{b}(\mathrm{i}(2 \pi a+$ $\left.\left.\mathrm{e}^{\mathrm{i} \theta} \delta\right)\right) \simeq \operatorname{sgn}(\theta) \mathrm{e}^{-\operatorname{sgn}(a-b) \frac{\mathrm{i} \pi}{4}} \sqrt{|4 \pi(a-b)|}$ if $\operatorname{sgn}(a)=\operatorname{sgn}(b)$ and $|b|<|a|$, and $\kappa_{b}(\mathrm{i}(2 \pi a+$ $\left.\left.\mathrm{e}^{\mathrm{i} \theta} \delta\right)\right) \simeq \mathrm{e}^{-\operatorname{sgn}(a-b) \frac{\mathrm{i} \pi}{4}} \sqrt{|4 \pi(a-b)|}$ otherwise.

\section{C.4 Integral of $\chi_{\emptyset}^{\prime \prime}(v) / \kappa_{a}(v)$ as an infinite sum}

The indefinite integral of $\chi_{\emptyset}^{\prime \prime}(v) / \kappa_{a}(v)$ can be rewritten as an infinite sum by expanding $\chi_{\emptyset}^{\prime \prime}(v)$ as in 162 and computing the integrals using (51) and 184. After careful treatment of the exchange between the integral and the infinite sum, one has

$$
\begin{aligned}
\int_{-\infty}^{\nu} \mathrm{d} v \frac{\chi_{\emptyset}^{\prime \prime}(v)}{\kappa_{a}(v)}=\lim _{M \rightarrow \infty}\left(-2 I_{0}(\nu)-\sqrt{\frac{2 M}{\pi}} \kappa_{a}(\nu)+\frac{\kappa_{a}^{2}(\nu)}{8}\right. \\
\left.+\sum_{b=-M+1 / 2}^{M-1 / 2} \log \left(1+\frac{\kappa_{a}(\nu)}{\kappa_{b}(\nu)}\right)\right),
\end{aligned}
$$

with $I_{0}$ defined in (68). When $\kappa_{a}(\nu)$ is small enough, i.e. when $\nu$ is in the vicinity of $2 \mathrm{i} \pi a$, the logarithm can be expanded. Using $2 I_{0}^{(m)}(\nu)=-\delta_{m, 1} / 4+(2 m-2) ! ! \sum_{b \in \mathbb{Z}+1 / 2} \kappa_{b}(\nu)^{-2 m}$ and $\chi_{\emptyset}^{(m+2)}(\nu)=-(2 m-1) ! ! \sum_{b \in \mathbb{Z}+1 / 2} \kappa_{b}(\nu)^{-2 m-1}$ for $m \geq 1$, we obtain the identity

$$
\int_{-\infty}^{\nu} \mathrm{d} v \frac{\chi_{\emptyset}^{\prime \prime}(v)}{\kappa_{a}(v)}=-\sum_{m=0}^{\infty}\left(\frac{\kappa_{a}(\nu)^{2 m}}{(2 m) ! !} 2 I_{0}^{(m)}(\nu)+\frac{\kappa_{a}(\nu)^{2 m+1}}{(2 m+1) ! !} \chi_{\emptyset}^{(m+2)}(\nu)\right) .
$$

\section{Calculations of some integrals depending on $(\nu, \mu) \in \mathbb{D}_{2}$}

In this appendix, we compute some integrals depending on two variables $\nu$ and $\mu$.

\section{D.1 Domain of analyticity of functions $\log \left(\mathrm{e}^{\mathrm{i} \theta}\left(\kappa_{a}(\nu)+\kappa_{b}(\mu)\right)\right)$}

Let $a, b \in \mathbb{Z}+1 / 2$ and $(\nu, \mu) \in \mathbb{D}_{2}$. We are interested in the position in the complex plane of $\kappa_{a}(\nu)+\kappa_{b}(\mu)$. Using (180), we obtain

$$
\arg \left(\mathrm{i}^{\frac{\operatorname{sgn}(a)-\operatorname{sgn}(b)}{2}}\left(\kappa_{a}(\nu)+\kappa_{b}(\mu)\right)\right) \in\left\{\begin{array}{cc}
(-3 \pi / 4, \pi / 4) & a<0 \quad b<0 \\
(-\pi / 4,3 \pi / 4) & a>0 \quad b>0 \\
(-3 \pi / 4,3 \pi / 4) & a b<0
\end{array}\right.
$$

for $\operatorname{Re} \nu<\operatorname{Re} \mu$,

$$
\arg \left(\mathrm{i}^{\operatorname{sgn}(b)-\operatorname{sgn}(a)} \frac{}{2}\left(\kappa_{a}(\nu)+\kappa_{b}(\mu)\right)\right) \in\left\{\begin{array}{cc}
(-3 \pi / 4, \pi / 4) & a<0 \quad b<0 \\
(-\pi / 4,3 \pi / 4) & a>0 \quad b>0 \\
(-3 \pi / 4,3 \pi / 4) & a b<0
\end{array}\right.
$$


for $\operatorname{Re} \nu>\operatorname{Re} \mu$, and

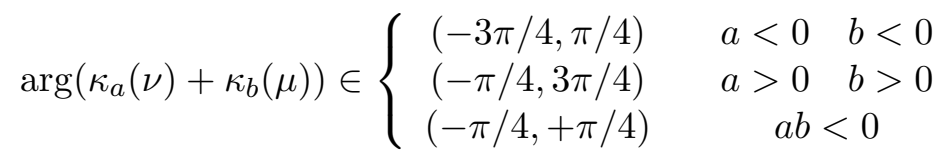

for $\operatorname{Re} \nu=\operatorname{Re} \mu, 0<\operatorname{Im}(\nu-\mu)<2 \pi$.

This implies in particular that the function $(\nu, \mu) \mapsto \log \left(\mathrm{i}^{\frac{\operatorname{sgn}(a)-\operatorname{sgn}(b)}{2}}\left(\kappa_{a}(\nu)+\kappa_{b}(\mu)\right)\right)$ is analytic in the domain $\{(\nu, \mu) \in \mathbb{D} \times \mathbb{D}, \operatorname{Re} \nu<\operatorname{Re} \mu\}$ while the function $(\nu, \mu) \mapsto$

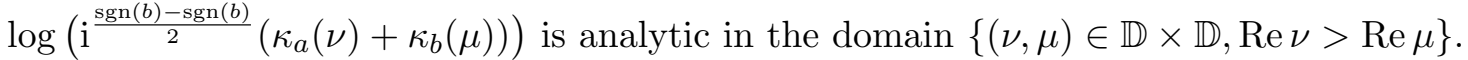

\section{D.2 Integral of $\kappa_{a}(u+\nu)^{-1} \kappa_{b}(u+\mu)^{-1}$}

Let $a, b \in \mathbb{Z}+1 / 2$. We consider the integral $\int_{-\Lambda}^{v} \mathrm{~d} u \kappa_{a}(u+\nu)^{-1} \kappa_{b}(u+\mu)^{-1}$ with a path of integration such that $(u+\nu, u+\mu)$ stays in $\mathbb{D}_{2}$ and $|\Lambda| \rightarrow \infty, \operatorname{Re} \Lambda>0$. The identity

$$
\frac{1}{\kappa_{a}(u+\nu) \kappa_{b}(u+\mu)}=-\partial_{u} \log \left(\mathrm{e}^{\mathrm{i} \theta}\left(\kappa_{a}(u+\nu)+\kappa_{b}(u+\mu)\right)\right)
$$

allows to compute the integral explicitly by choosing $\theta$ appropriately so that $\log \left(\mathrm{e}^{\mathrm{i} \theta}\left(\kappa_{a}(u+\right.\right.$ $\left.\left.\nu)+\kappa_{b}(u+\mu)\right)\right)$ is analytic everywhere on the path of integration, with $\mathbb{R}^{-}$the branch cut of the logarithm. According to appendix D.1, one can take $\theta=\theta_{a, b}(\mu-\nu)$ with

$$
\theta_{a, b}(z)=\pi \operatorname{sgn}(\operatorname{Re} z) \frac{\operatorname{sgn}(a)-\operatorname{sgn}(b)}{4} .
$$

Subtracting the leading term $\log \sqrt{\Lambda}$ in the limit $\Lambda \rightarrow \infty, \operatorname{Re} \Lambda>0$, the regularized integral is finally equal to

$$
f_{-\infty}^{v} \frac{\mathrm{d} u}{\kappa_{a}(u+\nu) \kappa_{b}(u+\mu)}=\mathrm{i} \theta_{a, b}(\mu-\nu)-\log \left(\mathrm{e}^{\mathrm{i} \theta_{a, b}(\mu-\nu)} \frac{\kappa_{a}(v+\nu)+\kappa_{b}(v+\mu)}{\sqrt{8}}\right) .
$$

This expression simplifies further when $v \rightarrow 2 \mathrm{i} \pi a-\nu$ since then $\kappa_{a}(v+\nu) \rightarrow 0$, and we obtain

$$
f_{-\infty}^{2 \mathrm{i} \pi a-\nu} \frac{\mathrm{d} u}{\kappa_{a}(u+\nu) \kappa_{b}(u+\mu)}=-\log \left(\frac{\kappa_{b}(\mu-\nu+2 \mathrm{i} \pi a)}{\sqrt{8}}\right) .
$$

\section{D.3 Integral of $\kappa_{b}(u+\mu) / \kappa_{a}(u+\nu)$}

Let $a, b \in \mathbb{Z}+1 / 2$. We consider the integral $\int_{-\Lambda}^{v} \mathrm{~d} u \kappa_{b}(u+\mu) / \kappa_{a}(u+\nu)$ with a path of integration such that $(u+\nu, u+\mu)$ stays in $\mathbb{D}_{2}$ and $|\Lambda| \rightarrow \infty$, $\operatorname{Re} \Lambda>0$. The identity

$$
\begin{aligned}
\frac{\kappa_{b}(u+\mu)}{\kappa_{a}(u+\nu)}=-\partial_{u}( & \frac{\kappa_{a}(u+\nu) \kappa_{b}(u+\mu)}{2} \\
& \left.+((\nu-2 \mathrm{i} \pi a)-(\mu-2 \mathrm{i} \pi b)) \log \left(\mathrm{e}^{\mathrm{i} \theta}\left(\kappa_{a}(u+\nu)+\kappa_{b}(u+\mu)\right)\right)\right)
\end{aligned}
$$

allows to compute the integral explicitly by choosing $\theta=\theta_{a, b}(\mu-\nu)$ defined in 192 so that $\log \left(\mathrm{e}^{\mathrm{i} \theta}\left(\kappa_{a}(u+\nu)+\kappa_{b}(u+\mu)\right)\right)$ is analytic everywhere on the path of integration. The contribution of the lower limit of the integral $-\Lambda$ is equal to

$$
\Lambda+((\nu-2 \mathrm{i} \pi a)-(\mu-2 \mathrm{i} \pi b)) \log \left(\sqrt{8 \Lambda} \mathrm{e}^{\mathrm{i} \theta_{a, b}(\mu-\nu)}\right)-\frac{\nu+\mu-2 \mathrm{i} \pi(a+b)}{2}
$$


1774

1775

1778

when $\Lambda \rightarrow \infty$. Defining the regularized integral by subtracting the divergent term $\Lambda+$ $\frac{(\nu-2 \mathrm{i} \pi a)-(\mu-2 \mathrm{i} \pi b)}{2} \log \Lambda$ finally leads to

$$
\begin{aligned}
& f_{-\infty}^{v} \mathrm{~d} u \frac{\kappa_{b}(u+\mu)}{\kappa_{a}(u+\nu)}=-\frac{\nu+\mu-2 \mathrm{i} \pi(a+b)}{2}-\frac{\kappa_{a}(v+\nu) \kappa_{b}(v+\mu)}{2} \\
& +((\nu-2 \mathrm{i} \pi a)-(\mu-2 \mathrm{i} \pi b))\left(\mathrm{i} \theta_{a, b}(\mu-\nu)-\log \left(\mathrm{e}^{\mathrm{i} \theta_{a, b}(\mu-\nu)} \frac{\kappa_{a}(v+\nu)+\kappa_{b}(v+\mu)}{\sqrt{8}}\right)\right) .
\end{aligned}
$$

\section{D.4 Integral of $\chi_{\emptyset}^{\prime \prime}(u+\mu) / \kappa_{a}(u+\nu)$}

Let $a \in \mathbb{Z}+1 / 2$. We consider the integral $\int_{-\infty}^{v} \mathrm{~d} u \chi_{\emptyset}^{\prime \prime}(u+\mu) / \kappa_{a}(u+\nu)$ with a path of integration such that $(u+\nu, u+\mu)$ stays in $\mathbb{D}_{2}$. Let $M$ be a positive integer, that will be taken to infinity in the end. Using (61), $\partial_{u} \zeta(s, u)=-s \zeta(s+1, u)$ and (51), one has

$$
\begin{aligned}
& \int_{-\infty}^{v} \mathrm{~d} u \frac{\chi_{\emptyset}^{\prime \prime}(u+\mu)}{\kappa_{a}(u+\nu)}=-\sum_{b=-M+1 / 2}^{M-1 / 2} f_{-\infty}^{v} \frac{\mathrm{d} u}{\kappa_{a}(u+\nu) \kappa_{b}(u+\mu)} \\
&-\frac{1}{2 \sqrt{\pi}} f_{-\infty}^{v} \mathrm{~d} u \frac{\mathrm{e}^{\mathrm{i} \pi / 4} \zeta\left(\frac{1}{2}, M+\frac{1}{2}+\frac{u+\mu}{2 \mathrm{i} \pi}\right)+\mathrm{e}^{-\mathrm{i} \pi / 4} \zeta\left(\frac{1}{2}, M+\frac{1}{2}-\frac{u+\mu}{2 \mathrm{i} \pi}\right)}{\kappa_{a}(u+\nu)} .
\end{aligned}
$$

The large $M$ asymptotics of the integral in the second line is dominated by the contributions $u \sim M$, for which (59) gives

$$
\begin{aligned}
& -\frac{1}{2 \sqrt{\pi}}\left(\mathrm{e}^{\mathrm{i} \pi / 4} \zeta\left(\frac{1}{2}, M+\frac{1}{2}+\frac{u+\mu}{2 \mathrm{i} \pi}\right)+\mathrm{e}^{-\mathrm{i} \pi / 4} \zeta\left(\frac{1}{2}, M+\frac{1}{2}-\frac{u+\mu}{2 \mathrm{i} \pi}\right)\right) \\
& =\frac{\kappa_{M}(u+\mu)-\kappa_{-M}(u+\mu)}{2 \mathrm{i} \pi}+\mathcal{O}\left(M^{-3 / 2}\right) .
\end{aligned}
$$

$$
\begin{gathered}
\int_{-\infty}^{v} \mathrm{~d} u \frac{\chi_{\emptyset}^{\prime \prime}(u+\mu)}{\kappa_{a}(u+\nu)}=\lim _{M \rightarrow \infty}\left(-M \log M+M\left(1-\log \frac{\pi}{2}\right)-\frac{\sqrt{2 M}}{\sqrt{\pi}} \kappa_{a}(v+\nu)\right. \\
\left.-\frac{\nu-\mu-2 \mathrm{i} \pi a}{4}-\sum_{b=-M+1 / 2}^{M-1 / 2}\left(\mathrm{i} \theta_{a, b}(\mu-\nu)-\log \left(\mathrm{e}^{\mathrm{i} \theta_{a, b}(\mu-\nu)} \frac{\kappa_{a}(v+\nu)+\kappa_{b}(v+\mu)}{\sqrt{8}}\right)\right)\right) .
\end{gathered}
$$

with $\theta_{a, b}$ defined in (192). This expression can be simplified further when $v \rightarrow 2 \mathrm{i} \pi a-\nu$ since $\kappa_{a}(v+\nu) \rightarrow 0$. Using (71), we obtain

$$
\int_{-\infty}^{2 \mathrm{i} \pi a-\nu} \mathrm{d} u \frac{\chi_{\emptyset}^{\prime \prime}(u+\mu)}{\kappa_{a}(u+\nu)}=-2 I_{0}(\mu-\nu+2 \mathrm{i} \pi a) .
$$

\footnotetext{
E Identities for the coefficients $W_{P}, W_{P}^{\Delta}$
In this appendix, we give some identities for the coefficients $W_{P}$ and $W_{P}^{\Delta}$ defined in (77)
and (94).

E Identities for the coefficients $W_{P}, W_{P}^{\Delta}$
In this appendix, we give some identities for the coefficients $W_{P}$ and $W_{P}^{\Delta}$ defined in 77 )
and (94).

E Identities for the coefficients $W_{P}, W_{P}^{\Delta}$
In this appendix, we give some identities for the coefficients $W_{P}$ and $W_{P}^{\Delta}$ defined in 77 )
and (94).
} 


\section{E.1 Differences of $W_{P}$}

We observe that for any $P \sqsubset \mathbb{Z}+1 / 2$, the last two terms in the definition (77) are unchanged if the set $P$ is replaced by $P-n, n \in \mathbb{Z}$. After some manipulations using $|P|_{+}+|P|_{-}=|P|,|P|_{+}-|P|_{-}=\sum_{a \in P} \operatorname{sgn}(a)$ and $\operatorname{sgn}(a-n)=\sigma_{a}\left(B_{n}\right) \operatorname{sgn}(a)$, one has

$$
W_{P}-W_{P-n}=-\mathrm{i} \pi n|P|+\operatorname{sgn}(n) 2 \mathrm{i} \pi|P|\left|P \cap B_{n}\right|,
$$

with $\operatorname{sgn}(0)=0$. Using this identity together with $\left|P \ominus B_{n}\right|=|P|+|n|-2\left|P \cap B_{n}\right|$ and $\left|\left(P \ominus B_{n}\right) \cap B_{n}\right|=|n|-\left|P \cap B_{n}\right|$, we obtain

$$
\left(W_{P \ominus B_{n}}-W_{P-n}\right)-\left(W_{P \ominus B_{n}-n}-W_{P}\right)=\operatorname{sgn}(n) \mathrm{i} \pi\left(|n|-2\left|P \cap B_{n}\right|\right)^{2},
$$

which can be rewritten as

$$
\begin{aligned}
& \left(W_{P \ominus B_{n}}-W_{P-n}\right)-\left(W_{\left(P \ominus B_{n}\right)-n}-W_{P}\right) \\
& \quad=\mathrm{i} \pi n+2 \mathrm{i} \pi \operatorname{sgn}(n)\left(\frac{|n|(|n|-1)}{2}-2|n|\left|P \cap B_{n}\right|+2\left|P \cap B_{n}\right|^{2}\right) .
\end{aligned}
$$

In particular, one has

$$
\left(W_{P \ominus B_{n}}-W_{P-n}\right)-\left(W_{P \ominus B_{n}-n}-W_{P}\right) \in \mathrm{i} \pi n+2 \mathrm{i} \pi \mathbb{Z} .
$$

\section{E.2 Ratios of $\mathrm{e}^{W_{P}}$}

We consider now the quantity $\mathrm{e}^{W_{P}}$. Using again $|P|_{+}^{2}-|P|_{-}^{2}=|P| \sum_{a \in P} \operatorname{sgn}(a)$, one has in terms of the Vandermonde determinant $V_{P}$ defined in (5) the identity

$$
\mathrm{e}^{W_{P}}=(-1)^{\frac{|P|(|P|-1)}{2}} \mathrm{e}^{-\mathrm{i} \pi \sum_{a \in P} a} \frac{(-1)^{|P|} V_{P}^{2}}{2^{2|P|}} .
$$

Considering ratios, one has in particular

$$
\mathrm{e}^{W_{P-n}-W_{P}}=(-1)^{n|P|},
$$

which follows also directly from 202).

We are also interested in the quantity $\mathrm{e}^{W_{P \ominus B_{n}}-W_{P}}$, which can be computed from 206 using the summation identity

$$
\sum_{a \in P \ominus Q} f(a)-\sum_{a \in P} f(a)=\sum_{a \in Q} \sigma_{a}(P) f(a),
$$

where $\sigma_{a}$ is defined in $(29)$. The summation identity $(208)$ is proved easily from the Venn diagram for the sets $P$ and $Q$. Applied to $Q=B_{n}, f(a)=a$, it gives after some simplifications for $P \sqsubset \mathbb{Z}+1 / 2$

$$
\mathrm{e}^{-\mathrm{i} \pi \sum_{a \in P \ominus B_{n}} a}=\mathrm{e}^{-\mathrm{i} \pi \sum_{a \in P} a} \times(-1)^{n(n+1) / 2} \mathrm{i}^{\sum_{a \in B_{n}} \sigma_{a}(P)} .
$$

Similarly, the summation identity $(208)$ implies $\left|P \ominus B_{n}\right|=|P|+\sum_{a \in B_{n}} \sigma_{a}(P)$, which leads to

$$
(-1)^{\frac{\left|P \ominus B_{n}\right|\left(\left|P \ominus B_{n}\right|-1\right)}{2}}=(-1)^{\frac{|P|(|P|-1)}{2}} \times \mathrm{i}^{-n}(-1)^{n|P|}(-1)^{n(n+1) / 2}(-\mathrm{i})^{\sum_{a \in B_{n}} \sigma_{a}(P)} .
$$

Putting together the two identities above with (206), we finally obtain

$$
\mathrm{e}^{W_{P \ominus B_{n}}-W_{P}}=\mathrm{i}^{-n}(-1)^{n|P|} \times \frac{(-1)^{\left|P \ominus B_{n}\right|} V_{P \ominus_{B_{n}}}^{2}}{2^{2\left|P \ominus B_{n}\right|}} / \frac{(-1)^{|P|} V_{P}^{2}}{2^{2|P|}} .
$$




\section{E.3 Ratios of $\mathrm{e}^{2 W_{P}^{\Delta}}$}

We consider the quantity $\mathrm{e}^{2 W_{P}^{\Delta}}$ with $W_{P}^{\Delta}$ defined in 94 . Similar simplifications as in the previous section give

$$
\mathrm{e}^{2 W_{(P+n) \ominus\left(B_{n} \backslash(\Delta+n)\right)}^{\Delta+n}-2 W_{P}^{\Delta}}=\frac{X_{(P+n) \ominus\left(B_{n} \backslash(\Delta+n)\right)}^{\Delta+n}}{X_{P}^{\Delta}}
$$

and

$$
\mathrm{e}^{2 W_{P \ominus\left(B_{n} \backslash \Delta\right)}^{\Delta}-2 W_{P-n}^{\Delta-n}}=\mathrm{e}^{2 W_{P \ominus\left(B_{n} \backslash \Delta\right)-n}^{\Delta-n}-2 W_{P}^{\Delta}}=\frac{X_{P \ominus\left(B_{n} \backslash \Delta\right)}^{\Delta}}{X_{P}^{\Delta}}
$$

with

$$
X_{P}^{\Delta}=(\mathrm{i} / 4)^{2|P \backslash \Delta|+|\Delta|} \prod_{a \in P \backslash \Delta} \prod_{\substack{b \in P \cup \Delta \\ b \neq a}}\left(\frac{2 \mathrm{i} \pi a}{4}-\frac{2 \mathrm{i} \pi b}{4}\right)^{2} .
$$

\section{References}

[1] T. Halpin-Healy and Y.-C. Zhang, Kinetic roughening phenomena, stochastic growth, directed polymers and all that. Aspects of multidisciplinary statistical mechanics, Phys. Rep. 254, 215 (1995), doi:10.1016/0370-1573(94)00087-J.

[2] T. Kriecherbauer and J. Krug, A pedestrian's view on interacting particle systems, KPZ universality and random matrices, J. Phys. A: Math. Theor. 43, 403001 (2010), doi: $10.1088 / 1751-8113 / 43 / 40 / 403001$.

[3] I. Corwin, The Kardar-Parisi-Zhang equation and universality class, Random Matrices: Theory and Applications 1, 1130001 (2011), doi:10.1142/S2010326311300014.

[4] A. Borodin and V. Gorin, Lectures on integrable probability (2012), https://arxiv. org/abs/1212.3351.

[5] J. Quastel and H. Spohn, The one-dimensional KPZ equation and its universality class, J. Stat. Phys. 160, 965 (2015), doi:10.1007/s10955-015-1250-9.

[6] T. Halpin-Healy and K. Takeuchi, A KPZ cocktail-shaken, not stirred..., J. Stat. Phys. 160, 794 (2015), doi:10.1007/s10955-015-1282-1.

[7] H. Spohn, The Kardar-Parisi-Zhang equation - a statistical physics perspective (2016), https://arxiv.org/abs/1601.00499.

[8] K. Takeuchi, An appetizer to modern developments on the Kardar-Parisi-Zhang universality class, Physica A 504, 77 (2018), doi:10.1016/j.physa.2018.03.009.

[9] A. Saenz, The KPZ universality class and related topics (2019), https://arxiv. org/abs/1904.03319.

[10] K. Takeuchi, M. Sano, T. Sasamoto and H. Spohn, Growing interfaces uncover universal fluctuations behind scale invariance, Sci. Rep. 1, 34 (2011), doi:10.1038/srep00034.

[11] A. Somoza, M. Ortuño and J. Prior, Universal distribution functions in two-dimensional localized systems, Phys. Rev. Lett. 99, 116602 (2007), doi:10.1103/PhysRevLett.99.116602. 
[12] H. van Beijeren, Exact results for anomalous transport in onedimensional Hamiltonian systems, Phys. Rev. Lett. 108, 180601 (2012), doi:10.1103/PhysRevLett.108.180601.

[13] H. Spohn, Nonlinear fluctuating hydrodynamics for anharmonic chains, J. Stat. Phys. 154, 1191 (2014), doi:10.1007/s10955-014-0933-y.

[14] V. Popkov, A. Schadschneider, J. Schmidt and G. Schütz, Fibonacci family of dynamical universality classes, Proc. Natl. Acad. Sci. USA 112, 12645 (2015), doi: $10.1073 /$ pnas. 1512261112 .

[15] M. Kulkarni, D. Huse and H. Spohn, Fluctuating hydrodynamics for a discrete GrossPitaevskii equation: Mapping onto the Kardar-Parisi-Zhang universality class, Phys. Rev. A 92, 043612 (2015), doi:10.1103/PhysRevA.92.043612.

[16] A. Nahum, J. Ruhman, S. Vijay and J. Haah, Quantum entanglement growth under random unitary dynamics, Phys. Rev. X 7, 031016 (2017), doi:10.1103/PhysRevX.7.031016.

[17] M. Ljubotina, M. Žnidarič and T. Prosen, Kardar-Parisi-Zhang physics in the quantum Heisenberg magnet, Phys. Rev. Lett. 122, 210602 (2019), doi:10.1103/PhysRevLett.122.210602.

[18] J. de Gier, A. Schadschneider, J. Schmidt and G. Schütz, Kardar-Parisi-Zhang universality of the Nagel-Schreckenberg model, Phys. Rev. E 100, 052111 (2019), doi:10.1103/PhysRevE.100.052111.

[19] M. Kardar, G. Parisi and Y.-C. Zhang, Dynamic scaling of growing interfaces, Phys. Rev. Lett. 56, 889 (1986), doi:10.1103/PhysRevLett.56.889.

[20] M. Hairer, Solving the KPZ equation, Ann. Math. 178, 559 (2013), doi: $10.4007 /$ annals.2013.178.2.4.

[21] A. Kupiainen, Renormalization group and stochastic PDEs, Annales Henri Poincaré 17, 497 (2016), doi:10.1007/s00023-015-0408-y.

[22] M. Gubinelli and N. Perkowski, KPZ reloaded, Commun. Math. Phys. 349, 165 (2017), doi:10.1007/s00220-016-2788-3.

[23] K. Johansson, Shape fluctuations and random matrices, Commun. Math. Phys. 209, 437 (2000), doi:10.1007/s002200050027.

[24] J. Baik and E. Rains, Limiting distributions for a polynuclear growth model with external sources, J. Stat. Phys. 100, 523 (2000), doi:10.1023/A:1018615306992.

[25] M. Prähofer and H. Spohn, Scale invariance of the PNG droplet and the Airy process, J. Stat. Phys. 108, 1071 (2002), doi:10.1023/A:1019791415147.

[26] T. Sasamoto, Spatial correlations of the 1D KPZ surface on a flat substrate, J. Phys. A: Math. Gen. 38, L549 (2005), doi:10.1088/0305-4470/38/33/L01.

[27] P. Ferrari and H. Spohn, Scaling limit for the space-time covariance of the stationary totally asymmetric simple exclusion process, Commun. Math. Phys. 265, 1 (2006), doi:10.1007/s00220-006-1549-0. 
[28] A. Borodin, P. Ferrari, M. Prähofer and T. Sasamoto, Fluctuation properties of the TASEP with periodic initial configuration, J. Stat. Phys. 129, 1055 (2007), doi:10.1007/s10955-007-9383-0.

[29] C. Tracy and H. Widom, Total current fluctuations in the asymmetric simple exclusion process, J. Math. Phys. 50, 095204 (2009), doi:10.1063/1.3136630.

[30] J. Baik, P. Ferrari and S. Péché, Limit process of stationary TASEP near the characteristic line, Comm. Pure Appl. Math. 63, 1017 (2010), doi:10.1002/cpa.20316.

[31] A. Borodin, I. Corwin and V. Gorin, Stochastic six-vertex model, Duke Math. J. 165, 563 (2016), doi:10.1215/00127094-3166843.

[32] K. Matetski, J. Quastel and D. Remenik, The KPZ fixed point (2017), https: //arxiv.org/abs/1701.00018.

[33] K. Johansson and M. Rahman, Multi-time distribution in discrete polynuclear growth (2019), https://arxiv.org/abs/1906.01053.

[34] Z. Liu, Multi-time distribution of TASEP (2019), https://arxiv.org/abs/1907. 09876 .

[35] C. Tracy and H. Widom, Level-spacing distributions and the Airy kernel, Commun. Math. Phys. 159, 151 (1994), doi:10.1007/BF02100489.

[36] B. Derrida and J. Lebowitz, Exact large deviation function in the asymmetric exclusion process, Phys. Rev. Lett. 80, 209 (1998), doi:10.1103/PhysRevLett.80.209.

[37] E. Brunet and B. Derrida, Probability distribution of the free energy of a directed polymer in a random medium, Phys. Rev. E 61, 6789 (2000), doi:10.1103/PhysRevE.61.6789.

[38] K. Mallick and S. Prolhac, Brownian bridges for late time asymptotics of KPZ fluctuations in finite volume, J. Stat. Phys. 173, 322 (2018), doi:10.1007/s10955-018-2136-4.

[39] S. Prolhac, Finite-time fluctuations for the totally asymmetric exclusion process, Phys. Rev. Lett. 116, 090601 (2016), doi:10.1103/PhysRevLett.116.090601.

[40] J. Baik and Z. Liu, Fluctuations of TASEP on a ring in relaxation time scale, Comm. Pure Appl. Math. 71, 0747 (2018), doi:10.1002/cpa.21702.

[41] Z. Liu, Height fluctuations of stationary TASEP on a ring in relaxation time scale, Ann. Inst. H. Poincaré Probab. Statist. 54, 1031 (2018), doi:10.1214/17-AIHP831.

[42] J. Baik and Z. Liu, Multi-point distribution of periodic TASEP, J. Amer. Math. Soc. 32, 609-674 (2019), doi:10.1090/jams/915.

[43] M. Gorissen, A. Lazarescu, K. Mallick and C. Vanderzande, Exact current statistics of the asymmetric simple exclusion process with open boundaries, Phys. Rev. Lett. 109, 170601 (2012), doi:10.1103/PhysRevLett.109.170601.

[44] L.-H. Gwa and H. Spohn, Six-vertex model, roughened surfaces, and an asymmetric spin Hamiltonian, Phys. Rev. Lett. 68, 725 (1992), doi:10.1103/PhysRevLett.68.725.

[45] S. Prolhac, Spectrum of the totally asymmetric simple exclusion process on a periodic lattice - first excited states, J. Phys. A: Math. Theor. 47, 375001 (2014). doi:10.1088/1751-8113/47/37/375001. 
1916

1917

1918

1919

1920

1921

1922

1923

1924

1925

[46] R. Blythe and M. Evans, Nonequilibrium steady states of matrix-product form: a solver's guide, J. Phys. A: Math. Theor. 40, R333 (2007), doi:10.1088/1751$8113 / 40 / 46 /$ R01.

[47] A. Lazarescu and K. Mallick, An exact formula for the statistics of the current in the TASEP with open boundaries, J. Phys. A: Math. Theor. 44, 315001 (2011), doi: $10.1088 / 1751-8113 / 44 / 31 / 315001$.

[48] T. Gautié, P. Le Doussal, S. Majumdar and G. Schehr, Non-crossing Brownian paths and Dyson Brownian motion under a moving boundary, J. Stat. Phys. (2019), doi:10.1007/s10955-019-02388-z.

[49] C. Bender and T. Wu, Anharmonic oscillator, Phys. Rev. 184, 1231 (1969), doi:10.1103/PhysRev.184.1231.

[50] T. Klassen and E. Melzer, The thermodynamics of purely elastic scattering theories and conformal perturbation theory, Nucl. Phys. B 350, 635 (1991), doi:10.1016/05503213(91)90159-U.

[51] P. Dorey and R. Tateo, Excited states by analytic continuation of TBA equations, Nucl. Phys. B 482, 639 (1996), doi:10.1016/S0550-3213(96)00516-0.

[52] A. Voros, The return of the quartic oscillator. The complex WKB method, Annales de l'I.H.P. Physique théorique 39, 211 (1983). http://www.numdam.org/item/AIHPA_ 1983_-39_3_211_0/.

[53] S. Prolhac, Current fluctuations and large deviations for periodic TASEP on the relaxation scale, J. Stat. Mech. 2015, P11028 (2015), doi:10.1088/17425468/2015/11/P11028.

[54] K. Motegi, K. Sakai and J. Sato, Long time asymptotics of the totally asymmetric simple exclusion process, J. Phys. A: Math. Theor. 45, 465004 (2012), doi:10.1088/1751$8113 / 45 / 46 / 465004$.

[55] E. Belokolos, A. Bobenko, V. Enolski, A. Its and V. Matveev, Algebro-geometric Approach in the Theory of Integrable Equations, Springer Series in Nonlinear Dynamics. Springer, Berlin, doi:10.1007/s00220-007-0235-1 (1994).

[56] O. Babelon, D. Bernard and M. Talon, Introduction to Classical Integrable Systems, Cambridge University Press, doi:10.1017/CBO9780511535024 (2003).

[57] A. Zabrodin, Lectures on nonlinear integrable equations and their solutions (2018), https://arxiv.org/abs/1812.11830.

[58] H. Fogedby, Nonequilibrium dynamics of a growing interface, J. Phys.: Condens. Matter 14, 1557 (2002), doi:10.1088/0953-8984/14/7/313.

[59] M. Janas, A. Kamenev and B. Meerson, Dynamical phase transition in large-deviation statistics of the Kardar-Parisi-Zhang equation, Phys. Rev. E 94, 032133 (2016), doi:10.1103/PhysRevE.94.032133.

[60] J. Baik and Z. Liu, TASEP on a ring in sub-relaxation time scale, J. Stat. Phys. 165, 1051 (2016), doi $10.1007 / \mathrm{s} 10955-016-1665-y$.

[61] N. Joshi, The second Painlevé hierarchy and the stationary KdV hierarchy, Publ. Res. Inst. Math. Sci. 40, 1039 (2004), doi:10.2977/prims/1145475502. 
[62] A. Zabrodin, Canonical and grand canonical partition functions of Dyson gases as taufunctions of integrable hierarchies and their fermionic realization, Complex Analysis and Operator Theory 4, 497 (2010), doi:10.1007/s11785-010-0063-8.

[63] J. Quastel and D. Remenik, KP governs random growth off a one dimensional substrate (2019), https://arxiv.org/abs/1908.10353.

[64] J. Feldman, H. Knörrer and E. Trubowitz, Riemann Surfaces of Infinite Genus, CRM Monograph Series 20. American Mathematical Society, Providence, doi $10.1090 / \mathrm{crmm} / 020$ (2003).

[65] A. Krajenbrink, P. Le Doussal and S. Prolhac, Systematic time expansion for the Kardar-Parisi-Zhang equation, linear statistics of the GUE at the edge and trapped fermions, Nucl. Phys. B 936, 239 (2018), doi:10.1016/j.nuclphysb.2018.09.019.

[66] P. Le Doussal, S. Majumdar, A. Rosso and G. Schehr, Exact short-time height distribution in the one-dimensional Kardar-Parisi-Zhang equation and edge fermions at high temperature, Phys. Rev. Lett. 117, 070403 (2016), doi:10.1103/PhysRevLett.117.070403.

[67] S. Prolhac, Tree structures for the current fluctuations in the exclusion process, J. Phys. A: Math. Theor. 43, 105002 (2010), doi:10.1088/1751-8113/43/10/105002.

[68] S. Prolhac, Perturbative solution for the spectral gap of the weakly asymmetric ex-

口 clusion process, J. Phys. A: Math. Theor. 50, 315001 (2017), doi:10.1088/17518121/aa77de.

[69] S. Prolhac, Extrapolation methods and Bethe ansatz for the asymmetric exclusion process, J. Phys. A: Math. Theor. 49, 454002 (2016), doi:10.1088/1751$8113 / 49 / 45 / 454002$.

[70] Z. Liu, A. Saenz and D. Wang, Integral formulas of ASEP and q-TAZRP on a ring (2019), https://arxiv.org/abs/1905.02987.

[71] R. Kenyon and D. Wilson, Critical resonance in the non-intersecting lattice path model, Probab. Theor. Rel. Fields 130, 289 (2004), doi:10.1007/s00440-003-0293-z.

[72] M. Poplavskyi, R. Tribe and O. Zaboronski, On the distribution of the largest real eigenvalue for the real Ginibre ensemble, Ann. Appl. Probab. 27, 1395 (2017), doi:10.1214/16-AAP1233.

[73] J. Baik and T. Bothner, The largest real eigenvalue in the real Ginibre ensemble and its relation to the Zakharov-Shabat system (2018), https://arxiv.org/abs/1808. 02419 .

[74] B. Derrida and A. Gerschenfeld, Current fluctuations of the one dimensional symmetric simple exclusion process with step initial condition, J. Stat. Phys. 136, 1 (2009), doi: $10.1007 / \mathrm{s} 10955-009-9772-7$.

[75] J.-M. Stéphan, Return probability after a quench from a domain wall initial state in the spin-1/2 XXZ chain, J. Stat. Mech. 2017, 103108 (2017), doi:10.1088/1742$5468 /$ aa8c19.

[76] E. Brattain, N. Do and A. Saenz, The completeness of the Bethe ansatz for the periodic ASEP (2015), https://arxiv.org/abs/1511.03762. 
[77] R. Langlands and Y. Saint-Aubin, Algebro-geometric aspects of the Bethe equations, In Strings and Symmetries, vol. 447 of Lecture Notes in Physics, pp. 40-53. Berlin: Springer, doi:10.1007/3-540-59163-X_254 (1995).

[78] R. Langlands and Y. Saint-Aubin, Aspects combinatoires des équations de Bethe, In Advances in Mathematical Sciences: CRM's 25 Years, vol. 11 of CRM Proceedings and Lecture Notes, pp. 231-302. Amer. Math. Soc., doi:10.1090/crmp/011 (1997).

[79] K. Motegi and K. Sakai, Vertex models, TASEP and Grothendieck polynomials, J. Phys. A: Math. Theor. 46, 355201 (2013), doi:10.1088/1751-8113/46/35/355201.

[80] K. Motegi and K. Sakai, K-theoretic boson-fermion correspondence and melting crystals, J. Phys. A: Math. Theor. 47, 445202 (2014), doi:10.1088/1751$8113 / 47 / 44 / 445202$.

[81] A. Bobenko, Introduction to Compact Riemann Surfaces, vol. 2013 of Lecture Notes in Mathematics, Springer, Berlin, Heidelberg, doi:10.1007/978-3-642-17413-1_1 (2013).

[82] R. Cavalieri and E. Miles, Riemann Surfaces and Algebraic Curves: A First Course in Hurwitz Theory, Cambridge University Press, doi:10.1017/CBO9781316569252 (2016).

[83] B. Eynard, Lectures notes on compact Riemann surfaces (2018), https://arxiv. org/abs/1805.06405.

[84] L. Beineke and F. Harary, The genus of the n-cube, Canadian Journal of Mathematics 17, 494 (1965), doi:10.4153/CJM-1965-048-6.

[85] G. Springer, Introduction to Riemann surfaces, Addison-Wesley (1957).

[86] O. Costin and S. Garoufalidis, Resurgence of the fractional polylogarithms, Math. Res. Lett. 16, 817 (2009), doi:10.4310/MRL.2009.v16.n5.a5.

[87] O. Golinelli and K. Mallick, Spectral gap of the totally asymmetric exclusion process at arbitrary filling, J. Phys. A: Math. Gen. 38, 1419 (2005), doi:10.1088/03054470/38/7/001.

[88] N. Bogoliubov, Determinantal representation of the time-dependent stationary correlation function for the totally asymmetric simple exclusion model, SIGMA 5, 052 (2009), doi:10.3842/SIGMA.2009.052.

[89] K. Motegi, K. Sakai and J. Sato, Exact relaxation dynamics in the totally asymmetric simple exclusion process, Phys. Rev. E 85, 042105 (2012), doi:10.1103/PhysRevE.85.042105.

[90] S. Prolhac, Asymptotics for the norm of Bethe eigenstates in the periodic totally asymmetric exclusion process, J. Stat. Phys. 160, 926 (2015), doi $10.1007 /$ s10955015-1230-0.

[91] V. Priezzhev, Exact nonstationary probabilities in the asymmetric exclusion process on a ring, Phys. Rev. Lett 91, 050601 (2003), doi:10.1103/PhysRevLett.91.050601.

[92] C. Tracy and H. Widom, Integral formulas for the asymmetric simple exclusion process, Commun. Math. Phys. 279, 815 (2008), doi:10.1007/s00220-008-0443-3. 